\title{
ELECTROMIGRATION OF HYDROGEN AND DEUTERIUM IN VANADIUM, NIOBIUM AND TANTALUM
}

\author{
Craig Leebens Jensen
}

Ph. D. Thesis submitted to Iowa State University

Ames Laboratory, DOE Iowa State University

This report NoTICE sponsored by the prepared as an account or wat Energy States nor the United Government. Neither the Energy, nor any of the United States Departher the contractors, subcontractors, employees, nor any of of any warranty, express ors, or their employees, meir intity or responsibility implied, or assumess, makes or usefulness of any informat the accuracy, process disclosed, or reprmation, apparatus, prodeness Ames, Iowa 50011 


\section{DISCLAIMER}

This report was prepared as an account of work sponsored by an agency of the United States Government. Neither the United States Government nor any agency Thereof, nor any of their employees, makes any warranty, express or implied, or assumes any legal liability or responsibility for the accuracy, completeness, or usefulness of any information, apparatus, product, or process disclosed, or represents that its use would not infringe privately owned rights. Reference herein to any specific commercial product, process, or service by trade name, trademark, manufacturer, or otherwise does not necessarily constitute or imply its endorsement, recommendation, or favoring by the United States Government or any agency thereof. The views and opinions of authors expressed herein do not necessarily state or reflect those of the United States Government or any agency thereof. 


\section{DISCLAIMER}

Portions of this document may be illegible in electronic image products. Images are produced from the best available original document. 
This report was prepared as an account of work sponsored by the United. States Government. Neither the United States nor the United States Department of Energy, nor any of their employees, nor any of their contractors, subcontractors, or their employees, makes any warranty, express or implied, or assumes any legal liability or responsibility for the accuracy, completeness, or usefulness of any information, apparatus, product or process disclosed, or represents that its use would not infringe privately owned rights:

Available from: National Technical Information Service

U. S. Department of Commerce

P.O. Box 1553

Springfield, VA 22161

Price: Microfiche $\$ 3.00$ 
Electromigration of hydrogen and deuterium in vanadium, niobium and tantalum

by

Craig Leebens Jensen

An Abstract of

A Dissertation Submitted to the Graduate Faculty in Partial Fulfillment of

The Requirements for the Degree of DOCTOR OF PHILOSOPHY

Approved :

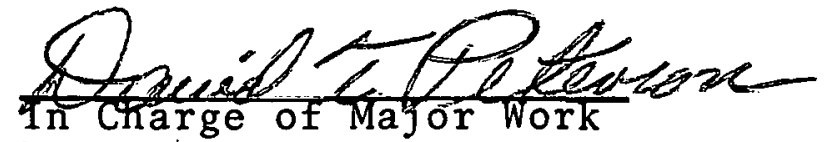

Por the Major Department

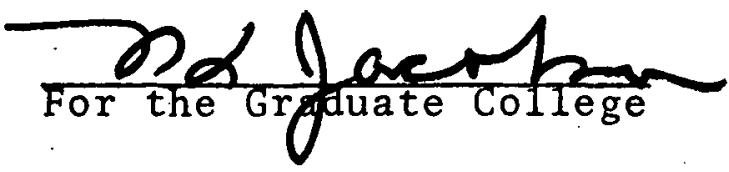

Iowa State University

Ames, Iowa 
TABLE OF CONTENTS

Page

INTRODUCTION $\quad 1$

$\begin{array}{ll}\text { EXPERIMENTAL METHOD } & 15\end{array}$

Resistance Technique for Electric Mobility 15

Measurement

Method of measurement $\quad 15$

Equipment $\quad 20$

Experimental technique 31

Steady-State Electromigration Experiments $\quad 38$

Method of measurement 38

Equipment $\quad 42$

Experimental procedure $\quad 45$

Electrical Resistivity Measurements 48

Electrical resistivity of niobium- 48 tantalum alloys

Hydrogen isotope electrical resistivity 50

Hydrogen Diffusivity Measurement 50

RESULTS 55

Sample Characterization $\quad 55$

Interstitial impurities 55

Alloy composition $\quad 56$

Density and atomic weight 56

Electric Mobility Measurements $\quad 57$

Steady-State Electromigration Measurements 65

Thermodynamic correction factor 65

Calculation of U/D 67

Vanadium 71

Niubium-tantalum alloys $\quad 72$

Niobium-Tantalum Alloy Resistivity 79

Hydrogen Isotope Resistivities $\quad 79$

Diffusivity of Hydrogen 83

Internal Consistency of the Experimental 92

Data for $U, D$ and $U / D$

$\begin{array}{ll}\text { DISCUSSION } & 96\end{array}$

CONCLUSIONS 112

ACKNOWLEDGEMENTS 114

$\begin{array}{ll}\text { B IBL IOGRAPHY } & 115\end{array}$ 
APPENDIX B. ELECTROLYTIC INTRODUCTION OF HYDROGEN ISOTOPES

APPENDIX C. TIME TO STEADY-STATE 
Electromigration of hydrogen and deuterium in vanadium, niobium and tantalum

by

Craig Leebens Jensen

An Abstract of

A Dissertation Submitted to the

Graduate Faculty in Partial Fulfillment of

The Requirements for the Degree of

DOCTOR OF PHILOSOPHY

Approved:
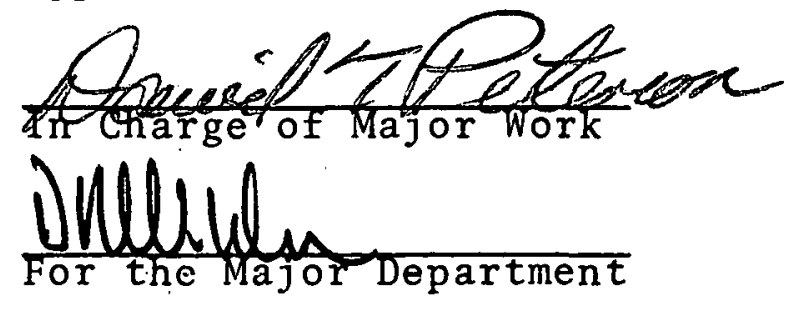

For the argaluate corlegen

Iowa State University

Ames, Iowa 
Electromigration of hydrogen and deuterium in vanadium, niobium and tantalum*

\section{Craig Leebens Jensen}

Under the supervision of D. T. Peterson

From the Department of Materials Science and Engineering Iowa State University

The electric mobility and effective valence of hydrogen and deuterium in vanadium, niobium, tantalum and three niobiumtantalum alloys were measured. A resistance technique was used to directly determine the electric mobility of hydrogen and deuterium at $30^{\circ} \mathrm{C}$ while a steady-state method was used to measure the effective valence. The use of mass spectrographic techniques on a single specimen which contained both hydrogen and deuterium greatly increased the precision with which the isotope effect in the effective valence could be measured. The effective valence for hydrogen isotopes was found to be positive for the pure metals and alloys with the possible exception of tantalum at solute concentrations below 1.5 atomic percent. The concentration dependence of the effective valence was small, except for tantalum where the effective valence decreases markedly below 3 atomic percent. In the niobium-tantalum alloy systems, the effective valence was

*USERDA Report IS-T-778. This work was performed under contract W-7405-eng-82 with the U.S. Energy Research and Development Administration. 
found to be independent of temperature and a linear function of alloy composition from $24^{\circ} \mathrm{C}$ to $120^{\circ} \mathrm{C}$. The isotope effect for the effective valence was generally small in contrast to the isotope effect in the electric mobility which exceeded the classically expected value.

The electrical resistivity of the niobium-tantalum alloys and the contribution of hydrogen isotopes to the electrical resistivity of these metals were measured at $22^{\circ} \mathrm{C}$. Electrical resistivity of the alloys obeyed the parabolic Nordheim relation for a binary alloy. The specific resistivity of hydrogen isotopes was found to be a linear function of alloy composition with the resistivity associated with hydrogen being greater than that for deuterium where an isotope effect was observed.

The diffusivity of hydrogen in the niobium-tantalum systems was measured as a function of hydrogen concentration at $23^{\circ} \mathrm{C}$. The diffusivity of hydrogen exhibited a negative deviation from a linear dependence on alloy composition. The diffusivity of hydrogen was found to decrease with hydrogen composition. 


\section{INTRODUCTION}

When a direct electric current is passed through a metallic conductor, macroscopic movement of interstitial, substitutional and intrinsic lattice atoms may occur. This phenomenon is usually called electromigration or, perhaps, electrotransport. Electromigration is analogous to ionic transport in solid electrolytes in that the presence of an electric field provides the driving force for atomic transport. The electric field superimposes only a slight gradient upon the potential energy-distance profile for a solute atom and thus, electromigration and electrolysis are very dependent on the diffusive character of the system.

Electromigration differs from transport in solid electrolytes in that the migrating atoms are not the primary charge carriers in the electromigration process. Thus, the effect of electron movement on the solute, as well as the electrostatic properties of the solute atom must be considered when examining electromigration driving forces. Because of the influence of electronic conduction on the solute combined with the role of diffusion, the electromigration process is dependent on the spatial, vibrational and electronic aspects of the 1 ocal defect. Electromigration studies may, therefore, provide insight into these basic processes occurring at the impurity site. Although the migrating atom is acted upon by a constant external force, the atom is not uniformly accelerated because 
of interactions between the solute and the surrounding lattice. Rather, the migrating atom moves through the crystal with a macroscopically constant velocity which is generally found to be proportional to the average electric field present. The ratio of the velocity of migration to the electric field is usually called the electric mobility, U.

Although the electric mobility provides a quantitative description of the velocity of migration, the electric mobility is not a true measure of the force felt by the migrating atom when an electric field is applied. It may be possible for an atom to be acted upon by a very large external force, yet have a very low migration velocity because of a high resistance to atomistic transport in the lattice. Conversely, in a lattice where resistance to atomistic transport is low, an atom may migrate rather rapidly even though the effective force on that atom may be sma11. Thus, there is a need to compensate the measured value of the electric mobility for varying resistance to atomic transport when discussing the force felt by a solute atom .

The quantity which describes the magnitude of the lattice resistance to transport of a specific solute is called the absolute mobility, B. The absolute mobility is defined as the ratio of the velocity of transport of a solute to the external$1 y$ applied force needed to attain that velocity. Thus, a high value for the absolute mobility of a particular solute indi- 
cates that the lattice has a high conductivity for atomic transport of that solute. Because the absolute mobility is a reflection only. of lattice resistance to atomic transport, the absolute mobility should not be a function of the transport process being investigated. Unfortunately, it is impossible to directly measure the absolute mobility: for any transport process, except perhaps diffusion. The difficulty arises in specifying the constant of proportionality relating the externally applied potential to the force produced on the migrating species.

The Einstein equation can, however, be used to express the absolute mobility in terms of diffusional transport as

$$
B=\frac{D(c)}{k T}\left[1+\frac{d \ln \gamma}{d \ln c}\right]^{-1}
$$

where $D(c)$ is the experimentally measured diffusion coefficient which may be a function of concentration, $k$ is Boltzmann's constant, $T$ is the absolute temperature and the term, d In $\gamma / d$ in $c$, describes the variation in activity coefficient with solute concentration. For the case of diffusional transport, difficulty arises in the determination of the absolute mobility because of solute-solute interactions which influence the measured diffusivity, $D(c)$. Thus, the $d$ in $\gamma / d$ in $c$ term is a reflection of the modification which must be made to correct the externally applied potential, in the form of a solute, concentration gradient, to the actual force on the solute 
determined by the gradient in chemical potential. If the activity coefficient is invariant, with concentration, the absolute mobility and, therefore, the lattice resistance to atomic transport may be determined directly by a measurement of the solute diffusivity.

For the case of electromigration, the force on the solute is generally assumed to be directly proportional to the magnitude of the applied electric field. The constant of proportionality relating the force and the electric field is usually called the effective charge, $e Z^{*}$, where $e$ is the fundamental unit of charge and $Z^{*}$, the effective valence, is the parameter to be determined by experiment. Combining the equations

$$
F=e Z * E
$$

and

$$
\mathrm{B}=\mathrm{v} / \mathrm{F},
$$

an expression for the effective charge can be written as

$$
Z * e=\frac{U}{B}
$$

where the electric mobility, $U$, has replaced v/E. Thus, $Z^{*} e$ represents the average velocity of the solute at a given electric field normalized by the atomistic conductivity of the 1attice. $Z^{*} e$ is a measure of the external force on a solute atom when a unit electric field is applied to the system. Equation 4 is not in a particularly useful form for calculation of $Z^{*}$ from experimental measurements. Substituting Equation 1 for the absolute mobility yields an expression for 
$2^{*}$ in terms of measurable quantities:

$$
Z^{*}=\frac{U k T}{D(c) e}\left[1+\frac{d \ln }{d \ln } c_{c}^{\gamma}\right]
$$

The thermodynamic correction factor, $d$ in $\gamma / d$ ln $c$, can be derived from equilibrium pressure-composition measurements. From the usual definition

$$
\gamma=\mathrm{P}_{\mathrm{H}_{2}}^{1 / 2} / \mathrm{C}
$$

where $\mathrm{C}$ is the concentration of hydrogen in the metal, the thermodynamic correction factor can be written as

$$
\frac{d \ln \gamma}{d \ln c}=\frac{d \ln P_{H_{2}}^{1 / 2}}{d \ln c}-1 .
$$

Equation 5 represents a link between the directly measurable quantity, electric mobility, and the fundamentally significant parameter, $Z^{*}$.

Although the expression assumed for the external force, Equation 2, appears to be based on an electrostatic model, the validity of Equation 5 depends only on the force being linearly related to the electric field. In this light, $Z^{*}$ must not be interpreted as the actual ionic state of the solute, but rather $Z^{*} e$ symbolizes the force felt by a solute at unit electric field.

Electromigration experiments have shown quite clearly that the force on a migrating atom must differ substantially from a purely electrostatic interaction. Experimentally meas - 
ured effective valences such as -22 for deuterium in silver (1) or +2 for hydrogen in niobium (2) may be taken as evidence for the existence of non-electrostatic forces. This is not a recent conclusion, but rather had been suggested in the early 1900 's as an explanation for the variation of migration direction of a given solute in different host metals. Skaupy (3) proposed that the charge carriers responsible for electronic conduction may transfer momentum to the solute atom. Wever and Seith (4) strengthened this postulate by making electromigration measurements on systems in which the majority charge carrier was known from Hall coefficient experiments. The result of these investigations indicated that the migration direction of the solute could, in most instances, be correlated with the sign of the majority charge carrier. Thus, it appears as if the moving charge carriers do exert a significant force on the solute atom.

Theoretical treatments have generally proceeded on the assumption that the driving force for electromigration can be separated into electrostatic and charge carrier-solute components. Those forces derived from charge carrier-solute interactions are generally called "electron wind" or "electron drag" forces. In regards to the electrostatic portion of the external force, there is disagreement as to whether a solute possessing an ionic charge can interact with the electric field. Bosvieux and Friedel (5) argue that due to screening 
of the impurity by the conduction electrons, the impurity cannot electrostatically feel the applied field. Huntington (6), however, reasons that slight perturbations of the screening charge with respect to the impurity site allow the solute to respond to the applied field. This controversy appears to have not been resolved, but rather has become a moot point due to the experimental difficulty in determining what types of forces are acting on the solute.

The component of force on the solute due to the presence of moving charge carriers has been calculated from two basic standpoints. Fiks (7), and later Huntington and Grone (8) have calculated the average momentum transferred to a solute as the result of ballistic interactions between charge carriers and the solute atom. Bosvieux and Friedel (5) employed a model based on local electronic polarization created near the impurity site by the moving charge carriers to calculate an average electrostatic force on the solute.

Although both treatments approach the electron drag force from quite different viewpoints, the predicted drag forces for each model are essentially equivalent. Both ballistic and polarization approaches predict an electron drag force which can be written as

$$
\mathrm{F}_{\mathrm{drag}}=z_{\mathrm{m}} \frac{\rho_{\mathrm{s}}}{\rho_{\mathrm{m}}}
$$

where $z_{m}$ is the number of free electrons per matrix atom and 
$\rho_{s} / \rho_{m}$ is the ratio of the specific resistivity of the solute to that of the host metal. $\rho_{\mathrm{s}} / \rho_{\mathrm{m}} \mathrm{can}$ be interpreted as the relative amount of the charge carrier scattering attributable to the solute participates.

Combining the electron drag force and any electrostatic component of the force, $F_{e l}$, which may be present, the total force on a solute due to the presence of an electric field may be written as

$$
\mathrm{F}_{\text {total }}=\mathrm{F}_{\mathrm{el}} \pm \mathrm{z}_{\mathrm{m}} \rho_{\mathrm{s}} / \rho_{\mathrm{m}}
$$

The sign of the electron drag term is determined by the sign of the majority charge carrier.

It should be noted that the electrostatic portion of the driving force for electromigration is dependent on the magnitude of the electric field, while the momentum transfer force is dependent on the current density. Unfortunately, Ohm's Law prohibits the independent variation of the electric field and current density so that the relative magnitude of the electrostatic and electron drag force is difficult to assess. One of the few methods by which the current density at a given electric field can be varied is by a temperature-induced resistivity change of the conductor. The only temperaturedependent portion of the electromigration force on a solute, as given by Equation 9 , is the quantity $\rho_{s} / \rho_{m}$. In most cases, the temperature variation of the matrix resistivity should be much greater than the temperature dependence of the solute 
resistivity. As the temperature is increased, $\rho_{s} / \rho_{m}$ should decrease and thus, the total force on the solute, as predicted by Equation 9, should vary as a function of temperature. The effective force, if extrapolated to infinite matrix resistivity, should yield the electrostatic contribution to the total electromigration force. Because electromigration experiments must be conducted at temperatures where the impurity is mobile, electromigration experiments involving solutes other than hydrogen are confined to high temperatures. Thus, the temperature range and therefore, the resistivity range over which electromigration experiments can be done is relatively small. Also, the precision associated inherently with electromigration measurements is usually not great, therefore, definitive interpretation of the extrapolation of effective force data as a function of temperature is not often possible.

The interstitial element, hydrogen, retains significant mobility down to liquid nitrogen temperatures and thus, the possibility for making electromigration measurements over a greater temperature range exists. In addition to extremely high mobility, hydrogen exhibits several other properties which simplify the experimental procedure and make knowledge of the electromigration behavior of hydrogen quite useful from theoretical and practical standpoints. Hydrogen and its stable isotope, deuterium, offer the greatest isotopic mass ratio. A knowledge of the mass dependence of the electric 
mobility and effective valence should be of great interest when assessing the relative roles of vibrational and electronic processes occurring at the local impurity site. $\mathrm{Be}-$ cause hydrogen isotopes are extremely mobile at ambient temperatures, relief is provided from the high temperature and high vacuum conditions necessary for other interstitial electromigration experiments. In vanadium, niobium and tantalum, hydrogen isotopes have high solubilities ranging from 0.02 to 0.10 hydrogen-to-metal atom ratio at $25^{\circ} \mathrm{C}$ (9). Coupled with the fact that hydrogen can be easily and reproducibly extracted from these metals by hot vacuum methods, the high solubility limits allow quite precise determination of hydrogen concentrations. Also, hydrogen can be introduced into vanadium, niobium and tantalum by rather convenient electrolytic methods.

Vanadium, niobium and tantalum provide interesting and practical host lattices for hydrogen isotopes. These metals, especially niobium and tantalum, are remarkably similar in many physical properties such as electrical resistivity and. atomic volume. Perhaps the largest difference between these metals is their atomic mass. Therefore, an examination of the electromigration properties of hydrogen in vanadium, niobium and tantalum may provide insight into the role of latticesolute vibrational coupling in interstitial transport.

Niobium and tantalum are so nearly identical that they form a continuous solid solution upon alloying. Niobium- 
tantaium alloys provide a means by which hydrogen diffusivity and electrical resistivity can be varied over a fairly wide range with changes in the electronic structure of the host which are probably small. The diffusivity of hydrogen decreases by a factor of four upon going from pure niobium to tantalum at $25^{\circ} \mathrm{C}(10)$. Thus, the effect of diffusive transport on the electromigration process may possibly be inferred from a study of the electromigration of hydrogen isotopes in niobium-tantalum alloys. Experimentally, it has been found that the resistivity of niobium-tantalum alloys rises to a value approximately $50 \%$ greater than the pure metal resistivities. In a sense, niobium-tantalum alloys provide a means by which the current density and electric field can be independ. ently varied over a much greater range than is possible with temperature-induced resistivity changes. This may provide a means by which the relative importance of the current density. dependent electron drag force and the electric field-dependent electrostatic contribution to the total force may be evaluated. Because of the possible application of niobium and vanadium in fusion technology, much emphasis has been placed on understanding the effects of impurities on the physical and mechanical properties of these metals. It is especially important to characterize the properties of hydrogen isotopes in niobium and vanadium because of the catastrophic embrittle= ment problems associated with dissolved hydrogen. In addition 
to providing information as to possible solute redistribution and concentration as a result of the presence of an electric field, electromigration investigations may provide basic knowledge concerning metal-hydrogen systems necessary for a complete characterization of interstitial effects.

Several methods have been employed to observe the electromigration of hydrogen isotopes. A widely employed technique, based on the measurement of steady-state flow rates of hydrogen gas from the metal, is primarily used at high temperatures where surface resistance is negligible. This method was first used to measure the effective valence of hydrogen isotopes in palladium $(11,12)$ and later applied to niobium, vanadium and tantalum (13), nickel (14), alpha-iron (14) and silver (1). Another technique has been used to measure the effective valence for hydrogen isotopes in vanadium, niobium and tantalum at lower temperatures (2). The effective valence of hydrogen isotopes was determined from a steady-state concentration profile established by balancing diffusive and electromigration fluxes when the ends of a cylindrical sample are rendered impermeable to hydrogen flux.

The results of these measurements indicate that the magnitude of the effective valence is rarely equal for hydrogen and deuterium. Perhaps more surprising than the nonclassical isotope effect is the range of values reported for the effective valence. As was noted, values for the effective 
valence range from -22 for deuterium in silver to near +3 for deuterium in niobium.

Taken as a whole, the electromigration of hydrogen isotopes in metals appears to follow the general trend of research involving hydrogen in metals. The'non-classical isotope effects reported in the effective valence of electromigration add to the long list of unexpected isotope effects observed for hydrogen-metal systems in such quantities as diffusivity (10), solubility (15) and molar volume (16). The study of hydrogen-metal systems has pointed out numerous deviations from classical behavior, yet there appears to be little consensus on how to account for the observed behavior. Attempts to reconcile theory with experiment range from the completely delocalized lattice-gas approach (17) to discrete quantum mechanical treatments (18). Unfortunately, perhaps much of the uncertainty as to a theoretical model stems from confusion in the experimentally observed results. Inconsistency in experiment and interpretation is probably due in part to the tremendous amount of research which has been done only recently in the area of metal-hydrogen systems. As in any area of science which has experienced rapid growth, inconsis tencies will tend to disappear over a period of years as a self-consistent picture of hydrogen in metals develops.

In order that further experimentation on hydrogen-metal systems aid, rather than retard the progress of this branch of 
science, it is necessary that experimental design provides checks on the internal consistency of the observed results. For the case of electromigration, two distinct types of exper:iments can be done. First, the electric mobility of hydrogen isotopes can be directly measured. Secondly, a steady-state technique can be used to measure the ratio of the electric mobility to solute diffusivity. Thus, if the diffusivity of the solute is known, the internal consistency of the experimental measurements of $U, D$ and $U / D$ can be evaluated.

This research will also involve the measurement of the resistivity of niobium-tantalum alloys, as well as the contribution to the resistivity made by hydrogen and deuterium. With resistivity data, comparison between calculated and measured driving forces of electromigration can be made. This systematic investigation of the electromigration of hydrogen isotopes in vanadium, niobium, tantalum and niobium tantalum alloys should aid in understanding basic processes occurring at the interstitial site as well as fundamental knowledge concerning interstitial transport. 
EXPERIMENTAL METHOD

Resistance Technique for Electric Mobility Measurement Method of measurement

Hydrogen isotopes increase the resistivity of Group VB metals and, therefore, it would be possible to determine hydrogen isotope concentration in various portions of a specimen by making local resistivity measurements. Alternatively, it is possible to determine the net flux of hydrogen isotopes into a region of a sample by measuring the resistance of that region as a function of time. If the experimental situation is reduced to cylindrical geometry and hydrogen is allowed to move through only one end of a specified section of the sample, then the total flux past a particular plane in the sample can be determined. This experimental geometry is shown in Figure 1 .

The presence of the double-step solute concentration profile indicated in Figure 1 will increase the resistance of the specimen by an amount related to the total quantity of solute. If the solute profile contains no concentration gradients, the resistance increase per unit length, $R_{S}$, will be constant along the length of the solute distribution. By placing resistance measurement probes on the sample as indicated in Figure 1, two sections of the sample have been defined which have solute fluxes through only one boundary of the section. As a direct current is passed through the specimen, the solute 


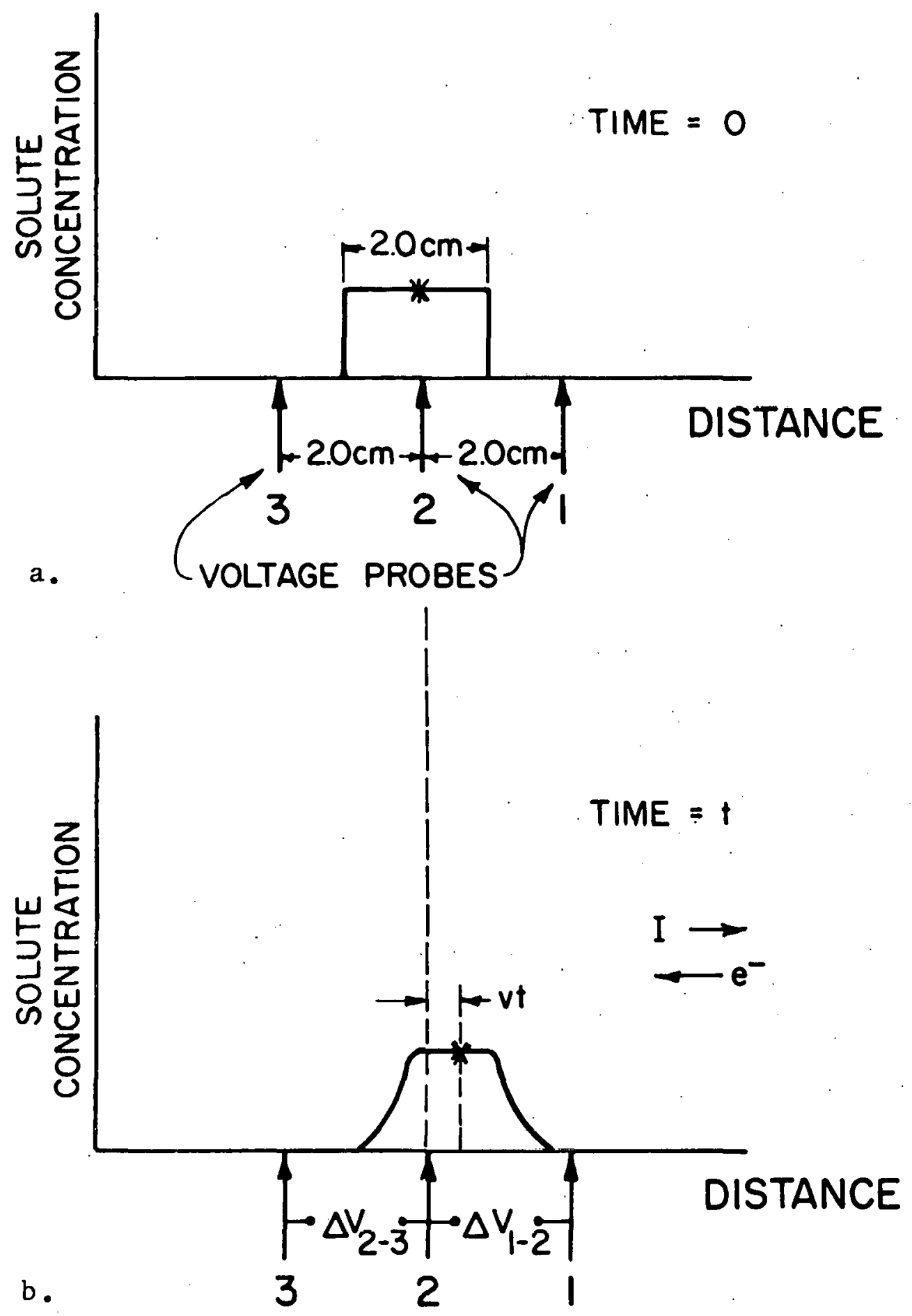

Figure 1. Schematic representation of solute profile; a. at time $=0, b$. at time $=t$ 
distribution will be displaced as a result of electromigration. Experimentally, it has been found that with the current direction as specified in Figure $1 b$, the hydrogen isotopes will drift with velocity, $v$, toward resistance probe 1 . After a time, $t$, the concentration profile will be displaced a distance $v \cdot t$. Because the amount of solute between probes 1 and 2 has now increased, the resistance of this section, $R_{1-2}$, will be increased by an amount

$$
\Delta \mathrm{R}_{1-2}=\mathrm{R}_{\mathrm{s}} \cdot \mathrm{v} \cdot \mathrm{t}
$$

If the solute distribution is uniform in the central portion of the concentration profile, $R_{1-2}$ should increase linearly with time. Therefore, Equation 10 can be written as:

$$
\frac{\mathrm{d} \Delta \mathrm{R}_{1-2}}{\mathrm{dt}}=\mathrm{v} \cdot \mathrm{R}_{\mathrm{s}} \text {. }
$$

Because the electromigration current is flowing through the sample, $\Delta \mathrm{R}_{1-2}$ can be continuously monitored by measuring the voltage drop along this section, $\Delta \mathrm{V}_{1-2}$, and the current flowing through the sample, I. If the electromigration current is constant,

$$
\frac{d \Delta V_{1-2}}{d t}=I \cdot \frac{d \Delta R_{1-2}}{d t}
$$

and the velocity of electromigration can be calculated by combining Equations 11 and 12 to give:

$$
v=\frac{d \Delta V_{1-2}}{d t} \cdot \frac{1}{I \cdot R_{S}} .
$$


The average electric field, E, can be calculated knowing the distance between probes 1 and 2 and $\Delta V_{1-2}$. The electric mobility, $U$, is then given by the ratio of $v$ to $E$.

Equation 13 should be valid for times such that no solute moves past probes 1 or 3 and for times such that there is no diffusional decrease in solute concentration at probe 2 . The first restriction on the experiment duration is necessary in order that the change in resistance of a section is due to only one flux and not the result of fluxes of different magnitudes leaving and entering opposite ends of the observed section. The second restriction on experiment length ensures that the initially measured value of $R_{S}$ corresponds to the solute concentration actually existing at probe 2 . As shown in Appendix A, the maximum experiment times calculated for each restriction are equal for the probe spacings and concentration profile length actually used. Maximum experiment times calculated in Appendix A range from 25 minutes for hydrogen in vanadium to 1350 minutes for deuterium in tantalum. The use of Equation 13 assumes that the solute profile contains no concentration gradients when $R_{S}$ is determined. This is necessary in order that the measured value of $R_{S}$ averaged over the entire solute distribution will be representative of the portion of the solute profile which moves past probe 2 during the electromigration experiment. Non-uniformity in the concentration profile near probe 2 can usually be detected due 
to the presence of a diffusion flux in addition to the electromigration flux. The existence of a diffusion flux near the center of the solute profile can be detected by reversal of the electromigration current. In one current direction, the electromigration and diffusive fluxes will tend to cancel, while with the current reversed, the two fluxes will be additive. Therefore, if the magnitude of $\mathrm{d}_{\Delta} \mathrm{V}_{1-2} / \mathrm{dt}$ varies with current direction, the presence of a solute concentration gradient in the central region of the hydrogen distribution is indicated.

Another assumption made in the use of Equation 13 for the calculation of electromigration velocities is that $R_{S}$ be a linear function of solute concentration. This is necessary in order that the total resistance change due to the presence of a fixed amount of solute does not vary as the shape of the solute distribution is modified by diffusion. If $R_{S}$ is not a linear function of hydrogen concentration, diffusive changes occurring at the ends of the solute profile would cause changes in $\Delta \mathrm{V}_{1-2}$ which are not the result of an electromigration flux. Because $R_{s}$ is measured within $6^{\circ} \mathrm{C}$ of the actual electromigration temperature, any error introduced by a possible temperature dependence of $R_{s}$ should be negligible.

It is necessary to maintain the sample at constant temperature since resistance changes in the specimen will occur if the sample temperature varies. Because the duration of the 
experiment is chosen such that no solute may move past probes 1 and 3 , the resistance of the specimen between probes 1 and 3 should remain constant if no temperature changes have occurred. This fact is used to verify that no temperature-induced resistance changes have been interpreted as resistance changes due to the electromigration flux. Experimentally, it has been found necessary to measure $\Delta V_{1-2}$ to $\pm 1 \times 10^{-5}$ volt in order that $\mathrm{d} \Delta \mathrm{V}_{1-2} / \mathrm{dt}$ be accurately determined within the time limit of the experiment. At a current density of $3000 \mathrm{amps} / \mathrm{cm}^{2}$ and a resistance probe spacing of $2.0 \mathrm{~cm}$, the maximum permissible temperature-induced change in specimen resistivity can be calculated as:

$$
\Delta \rho=1 \times 10^{-5} \mathrm{volt} /\left(2.0 \mathrm{~cm} \cdot 3 \times 10^{3} \mathrm{amps} / \mathrm{cm}^{2}\right)=1.7 \times 10^{-9} \mathrm{ohm}-\mathrm{cm} .
$$
Using a temperature coefficient of resistance of 0.004 per ${ }^{\circ} \mathrm{C}$ and a resistivity of $14 \times 10^{-6} \mathrm{ohm}-\mathrm{cm}$ for niobium, the maximum permissible temperature variation can be calculated as:

$$
\begin{aligned}
\Delta \mathrm{T} & =1.7 \times 10^{-9} \mathrm{ohm}-\mathrm{cm} /\left(14 \times 10^{-6} \mathrm{ohm}-\mathrm{cm} \cdot 4 \times 10^{-3} \text { per }{ }^{\circ} \mathrm{C}\right) \\
& =0.03^{\circ} \mathrm{C}
\end{aligned}
$$

The choice of a temperature range for the electromigration experiment is dictated by the desire to obtain maximum solute displacement by electromigration with minimal diffusive modification of the solute profile. It is, therefore, beneficial to perform the electromigration experiment at as low a temperature as can be achieved with a high current density. 


\section{Equipment}

Direct currents used for the measurement of $R_{S}$ and for the electromigration driving force were supplied by an externally programmed power supply operating in a constant current mode. With external programming control, it was possible to switch rapidly to preset currents while maintaining good current regulation. Therefore, $R_{s}$ could be measured using a small current to avoid joule heating of the sample while the predetermined electromigration current was directly accessible through a simple switching procedure. In accordance with the power supply manufacturer's instructions, resistances used to provide a voltage feedback for the external programming control were chosen to provide a 0.5 volt signal at maximum current. The minimum regulated current was approximately ten times smaller than the maximum regulated current. Several switch selectable feedback resistors were used to provide a regulated current range extending from 0.05 amp to 14 amps. After warm-up, current stability was usually better than $0.02 \%$ for the duration of the electromigration experiment. All currents were measured by observing the voltage drop across a $0.01 \mathrm{ohm}$ standard resistance. Switching was provided to reverse the current flow during the electromigration experiment for detection of solute profile non-uniformity. A schematic representation of the power supply, external programming and switching is shown in Figure 2 . 


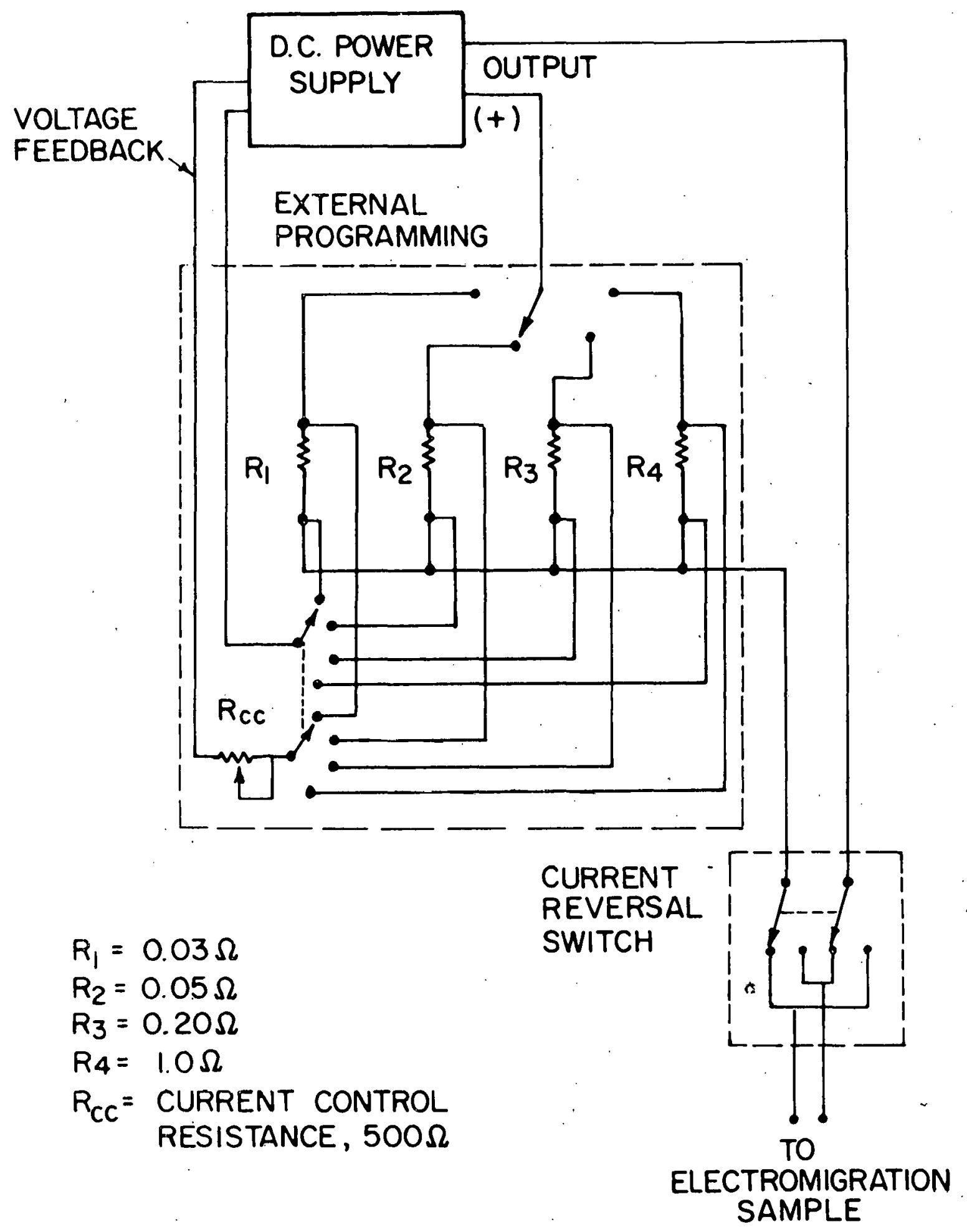

Figure 2. Power supply operating in constant current mode with external programming and current reversal switch 
All voltage measurements were made with a precision potentiometer capable of resolving $1 \times 10^{-6}$ volt. A11 incoming potentials were routed to the potentiometer by a rotary switch designed to generate minimal EMF as a result of switching.

Because of the relatively high current densities needed to achieve measurable solute displacement within the time limit of the experiment, it was necessary to dissipate approximately 3 watts of power along the $10 \mathrm{~cm}$ sample. To remove heat from the sample at a rapid rate, the sample holder was immersed in a rectangular metal tank which contained 20 1iters of mineral oil.

An outlet in the lower portion of the tank was connected to a magnetically-driven impeller pump. The return oil line was connected to the bottom of the sample holder and allowed the mineral oil to flow upward through a $6.0 \mathrm{~cm} \times 0.3 \mathrm{~cm} \mathrm{slot}$ in a bakelite sheet. The electromigration sample was placed on the bakelite sheet over the longitudinal slot. This arrangement provided a fairly uniform, lamellar oil flow past the central region of the sample at a rate of 0.33 liter per second. It was necessary to use a magnetically-driven impellar pump to reduce the amount of air drawn in around the shaft of conventional impeller pumps. An excessive amount of dis. solved air in the mineral oil aided the formation of gas bubbles in the low pressure region on top of the sample. These bubbles seriously interfered with heat transfer from 
the sample to the oil causing unacceptable variations in sample temperature. Periodical1y, absorbed gases were removed by placing the oil in a vacuum flask and reducing the pressure over the oil by means of a mechanical vacuum pump. Magnetic stirring greatly aided the release of entrained gases from the oil. It was also necessary to filter the mineral oil to remove lint which tended to collect on the sample. Filtering was done continuously by placing an 80 mesh stainless steel screen over the mineral oil intake.

The temperature of the sample was measured by spotwelding three chromel-alumel thermocouples to a $5.0 \mathrm{~cm}$ central section of a specimen operating under oil flow rates and oil bath temperatures identical to those used for the electro. migration experiments. Thermocouple wire of $0.10 \mathrm{~mm}$ diameter was used to minimize the effects on sample temperature due to the presence of the thermocouples. In practice, it is impos: sible to spot-weld both thermocouple leads to a single point on the sample. Because of this, an additional EMF will appear across the thermocouple when current is passed through the sample. This EMF is due to the voltage gradient between the thermocouple leads. The current-induced error in the thermocouple EMF can be removed if the measured thermocouple EMF is averaged over both current directions. The sample temperature was found to increase as the square of the electromigration current. At a current density of $3000 \mathrm{amps} / \mathrm{cm}^{2}$, the tempera- 
ture of the sample was found to increase approximately $6^{\circ} \mathrm{C}$ above the bath temperature. The longitudinal temperature variation along the $5.0 \mathrm{~cm}$ central section of the sample was less than $0.5^{\circ} \mathrm{C}$.

Because of the large amount of heat generated from the kinetic energy of the oil flow, it was necessary to install cooling coils in the oil bath to prevent the oil temperature from continually rising. Copper tubing was placed around the perimeter of the oil tank and several coils were also placed around the mineral oil intake. The rate of water flow through the coils was not varied during the electromigration experiment. A stirrer was placed in the oil tank to prevent the formation of long-range temperature gradients in the oil. The mineral oil flow system is represented in Figure 3.

Although the oil flow system did cool the sample to a reasonable level, temperature fluctuations were greater than could be tolerated for the electromigration measurements. To provide sufficient temperature control, the power supplied to a small heater located in the oil flow was varied in response to changes in the oil flow temperature. Although the voltage along the electromigration specimen was used to verify the absence of temperature changes, the electromigration sample was not used as the sensor for the temperature control system. A separate sensor was used to monitor the specimen temperature due to the difficulty in connecting the electromigration sample 
TOP VIEW

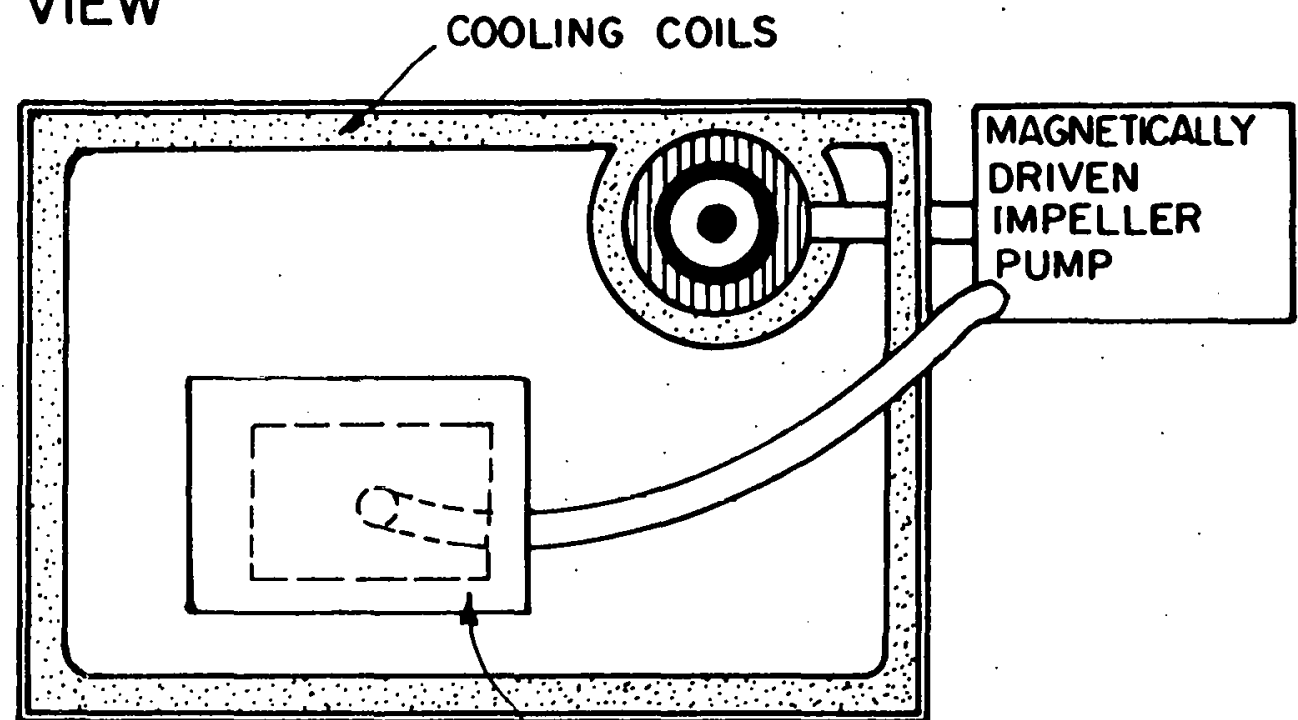

SAMPLE HOLDER

MINERAL

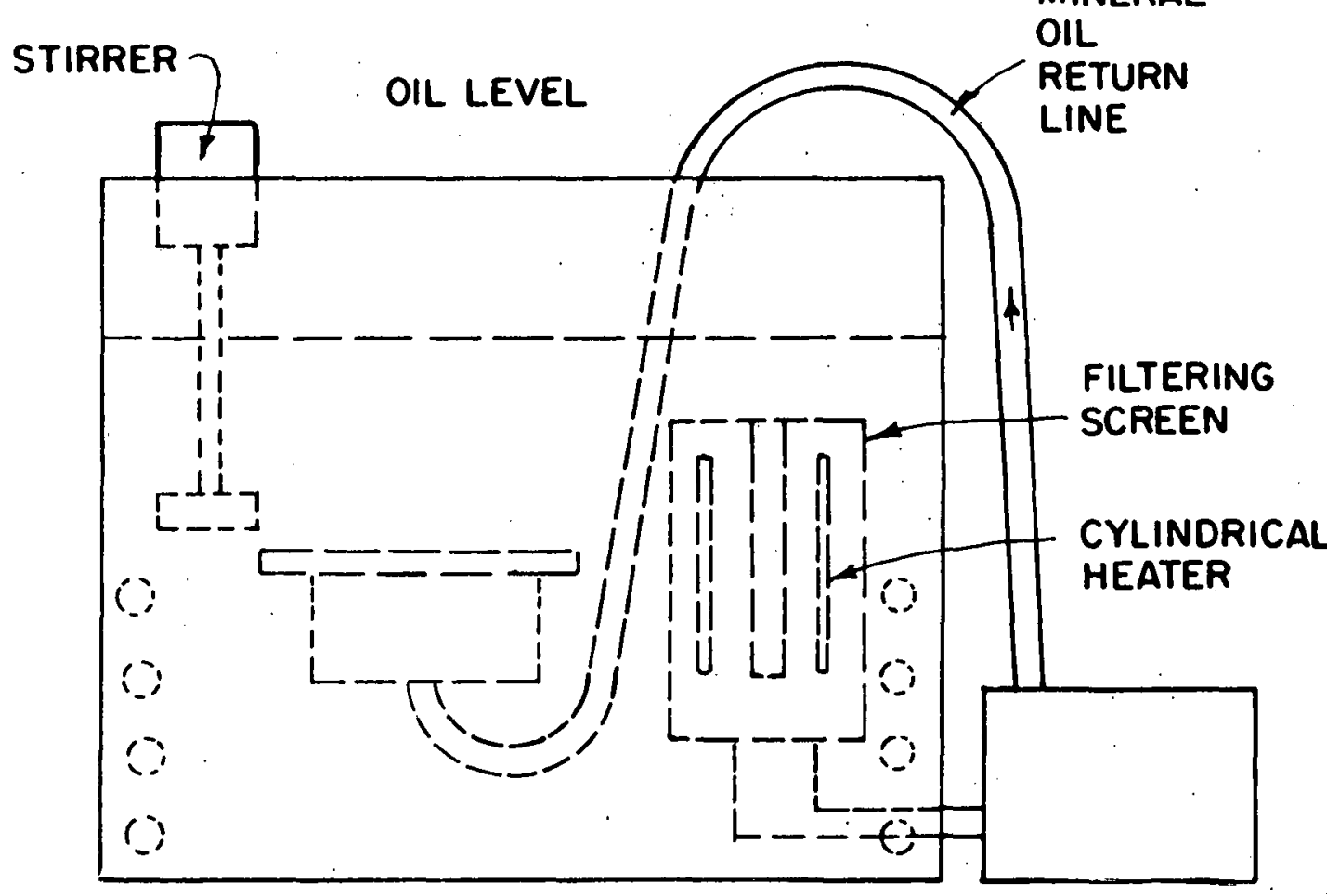

FRONT VIEW

Figure 3. Mineral oil tank and oil flow system 
to the temperature control system in a uniform manner in a short amount of time. A wire of the same size and material as the electromigration sample connected in series with the electromigration sample was used to sense changes in temperature. The temperature sensing wire was placed across the slot in the specimen holder directly below the electromigration sample. Two voltage probes were spot-welded $4 \mathrm{~cm}$ apart in the central section of the temperature sensing wire. With constant current, variations in the voltage between the probes will be a function of the temperature only. The voltage between the probes was placed across a voltage divider to obtain an output voltage of near $30 \mathrm{mV}$. The resistors were chosen such that the total resistance of the voltage divider would prevent excessive current flow through the voltage divider. However, the output voltage of the divider was used as the input for a proportional controller and the sensitivity of the controller varied inversely with the resistance of the source of the input potential. Thus, the resistances comprising the voltage divider could not be excessively large. The voltage divider used for the electromigration experiments is shown in Figure 4. The proportional controller, which varied the output of a variac in response to changes in the input signal, was a 0 $1200^{\circ} \mathrm{C}$ furnace controller modified to a range of $1 \mathrm{mV}$ for full scale deflection. This modification was done by the instrumentation Group of the Ames Laboratory. To decrease the 


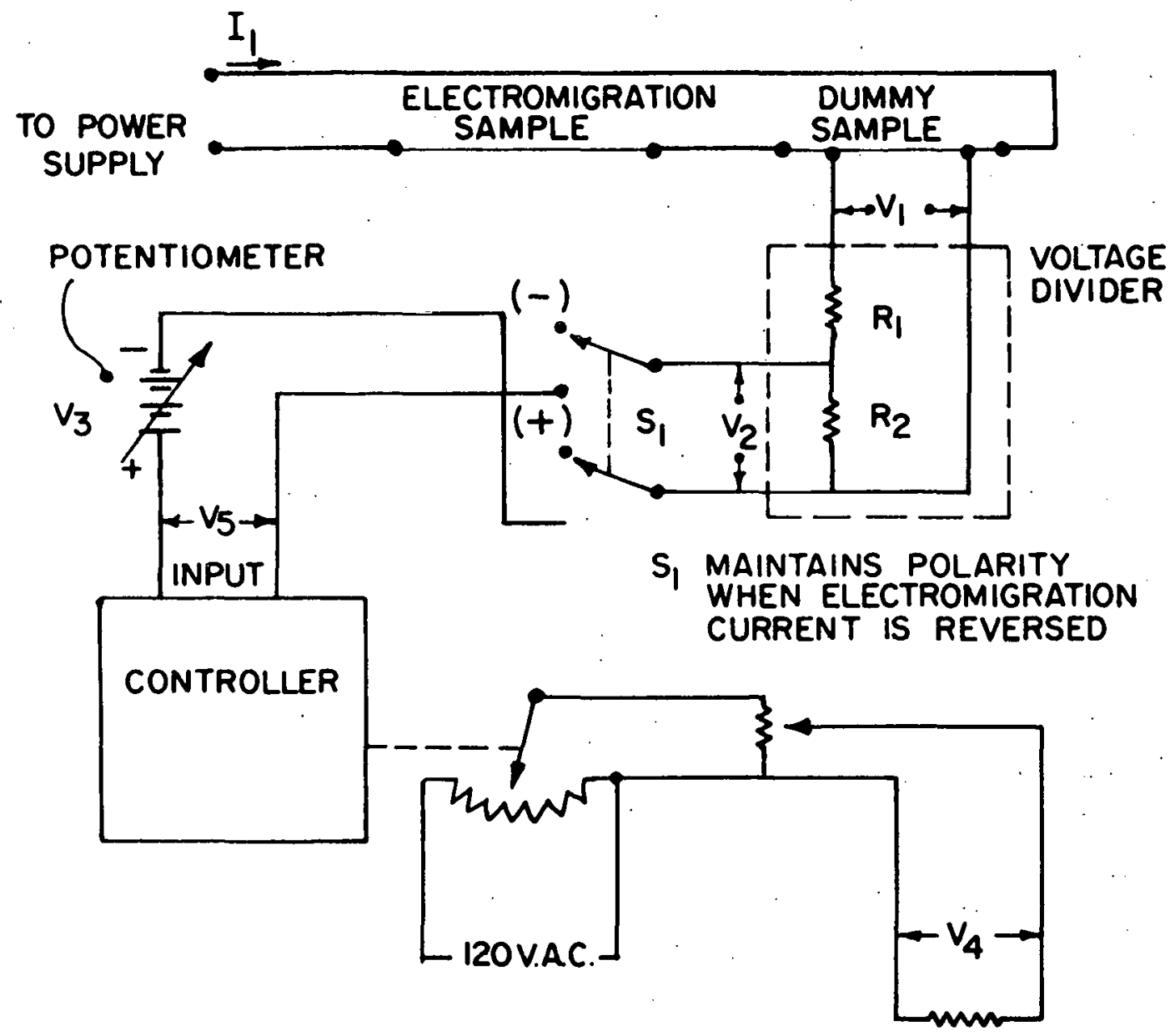

NORMAL OPERATING PARAMETERS

HEATER IN

OIL FLOW

$I_{1}=6$ AMPS D.C.

$V_{1}=250 \mathrm{mV} . D . C$.

$V_{2}=30 \mathrm{mV} \cdot D . C$.

$V_{3}=29.5 \mathrm{mV}$.D.C.

$V_{4}=25$ V.A.C.

$V_{5}=0.5 \mathrm{~m} \cdot$ V.D.C.

$R_{1}=70 \Omega$

$R_{2}=10 \Omega$

Figure 4. Temperature controi system 
output from the voltage divider to less than $1 \mathrm{mV}$ while maintaining the magnitude of temperature-induced voltage fluctua. tions, a potentiometer was connected in series with the voltage divider and controller. The output EMF of the potentiometer was opposed to the voltage across the divider, but approximately $0.5 \mathrm{mV}$ smaller. Therefore, the voltage seen by the controller was. reduced to $0.5 \mathrm{mV}$, while the magnitude of the temperature-induced voltage changes remains constant. For example, if a temperature-induced change of $0.01 \%$ in the output voltage of the divider was to appear, the signal seen by the controller would vary by $3 \%$ of the full scale deflection. This change can easily be compensated for by the controller. Using the nominal voltages indicated in Figure 4 for a current density of $3000 \mathrm{amps} / \mathrm{cm}^{2}$ along with the resistivity and temperature coefficient of resistance for niobium, a $0.01 \%$ variation in the voltage divider output can be shown to correspond to a $0.03^{\circ} \mathrm{C}$ change in sample temperature. Therefore, the control system was able to provide the necessary temperature stability for the electromigration measurements. The output from the servo-controlled variac was used as the input for another variac. This allowed the operator to compensate for small drifts in the control system. The output from the second variac was applied to a 720 watt heater in the mineral oil intake. Assuming a specific heat for mineral oil of 0.40 cal $1 / \mathrm{cm}^{3}-{ }^{\circ} \mathrm{C}$, the oil flow rate can be used to express the tem- 
perature rise of the oil, $\Delta \mathrm{T}$, as a function of the voltage supplied to the heater, $V$

$$
\Delta \mathrm{T}\left({ }^{\circ} \mathrm{C}\right)=7.3 \times 10^{-5} \mathrm{~V}^{2}
$$

It can be seen from Equation 14 that the best control of the oil temperature is obtained at low heater voltages. A one volt fluctuation in the heater voltage will cause a smaller temperature change at a low voltage in comparison with a similar fluctuation at a higher heater voltage. Normal heater voltage for the electromigration experiment was 25 volts. The temperature control system is schematicaliy represented in Figure 4 .

Because of the rather complex nature of the equilibrium temperature established by the oil flow, cooling coils and temperature control system, it was necessary to warm-up the electromigration apparatus for at least 30 minutes prior to the actual electromigration experiment. The warm-up period also improved the stability of the controller, output EMF of the potentiometer and the electromigration current.

The resistance measurement probes were made from brass strips ground to a knife-edge. The knife-edges were recessed into the bakelite sample holder and spanned the oil flow oriface. Two additional brass knife-edges were placed on top of the electromigration sample to insure good electrical contact between the sample and the lower knife edges. The oil flow tube from the pump was coupled to a rectangular manifold which 
was mounted below the bakelite sheet used to support the sample. The temperature control wire used as the sensor for the control system was mounted inside this manifold. The sample holder is represented in Figure 5. Experimental technique

Wire samples of niobium, vanadium and tantalum were prepared by arc-melting, swaging and drawing to $0.51 \mathrm{~mm}(0.020$ inch) diameter. The samples were cut to $12 \mathrm{~cm}$ lengths and fully annealed near $1700^{\circ} \mathrm{C}$ for 20 minutes in a vacuum of better than $1 \times 10^{-5}$ torr. The samples were heated by passing an alternating current through them. Sample temperature was estimated with an optical pyrometer. Copper wires of $0.25 \mathrm{~mm}(0.010$ inch) diameter were spot-welded approximately $5 \mathrm{~cm}$ apart in the central section of each sample. These leads were used for the measurement of the resistance increase of the specimen due to the presence of hydrogen isotopes. The copper leads were coated with an insulating lacquer as protection from electrolytic attack during electropolishing. Immediately preceding the electromigration experiment, the specimen was given a light electropolish to prepare the surface for electrolytic charging. After electropolishing, the electromigration sample was connected in series and placed in close proximity with a hydrogen-free dumm sample in a mineral oil bath. The oil bath was used to provide thermal inertia against variations in room temperature. The resistance of the central portion of both 

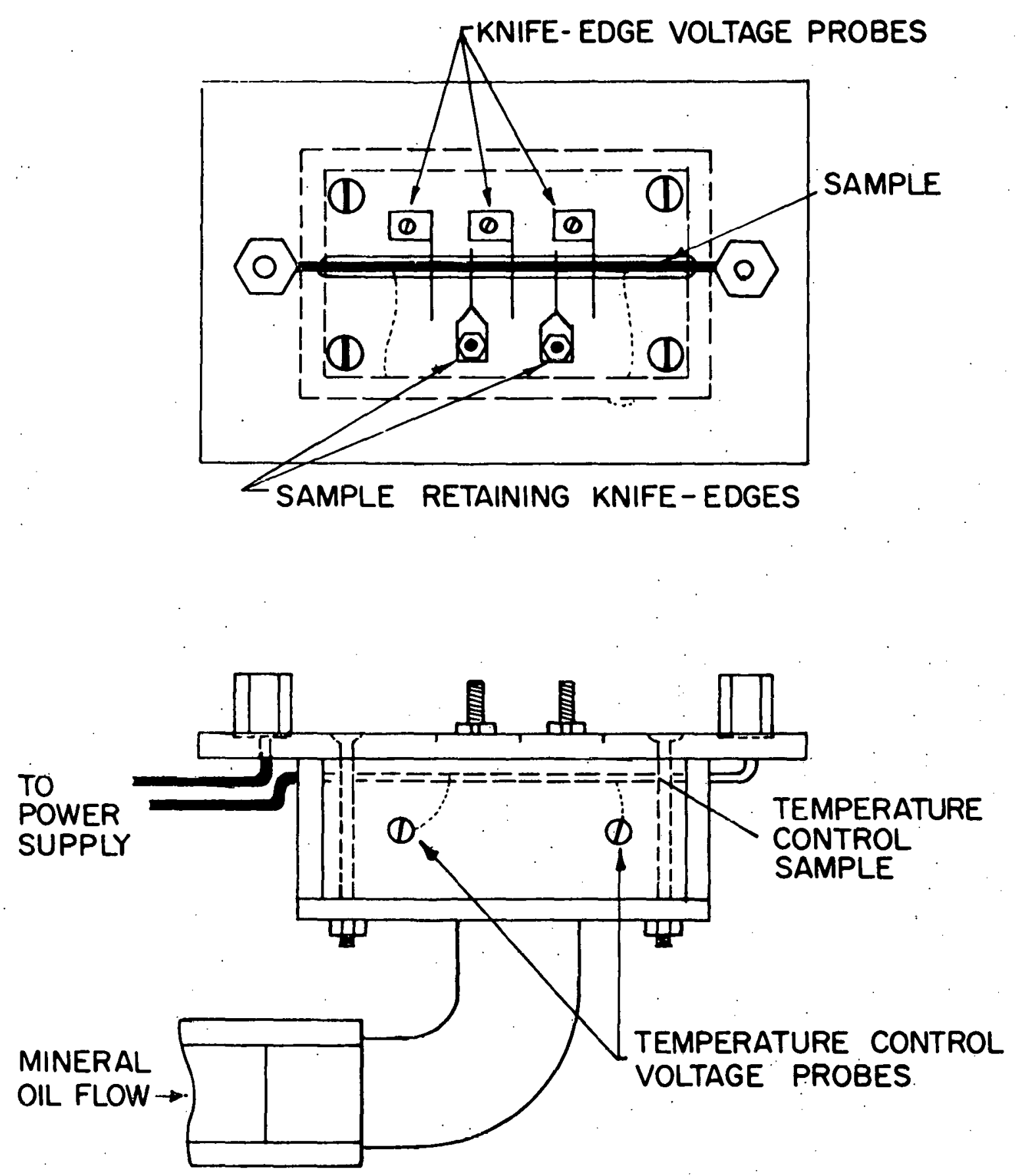

Figure 5. Electromigration specimen holder 
samples was determined by noting the voltage drop between the copper probes and the current flowing through the samples. The electromigration sample was then removed from the oil bath and cleaned in acetone followed by a methyl alcohol rinse. The electromigration specimen was then placed in the lower portion of the lucite cylinder shown in Figure 6. The top cylinder was temporarily secured to the lower portion by means of a thin layer of vacuum grease. The vacuum grease allowed the cylinders to be filled with the charging electrolyte so that only a $2.0 \mathrm{~cm}$ portion of the specimen contacted the electrolyte. The sample was then electrolytically charged with hydrogen or deuterium. If possible, a very light anodic layer was then deposited on the surface to mark the location of the charged section. The procedure for electropolishing, electrolytic charging and anodizing is fully described in Appendix B. The sample was then removed from the lucite cylinders and the vacuum grease was removed from the surface of the specimen with acetone. After replacing the electromigration sample in the mineral oil bath, the resistances of the hydrogen-charged specimen and the dummy sample were again measured. Due to the small sample diameter and the high diffusivities of hydrogen isotopes, radial homogeneity in the solute concentration was obtained very quickly. Thus, there was no need to wait an extended period of time before making the final resistance measurement. The solute resistance per unit length, $R_{S}$, could 


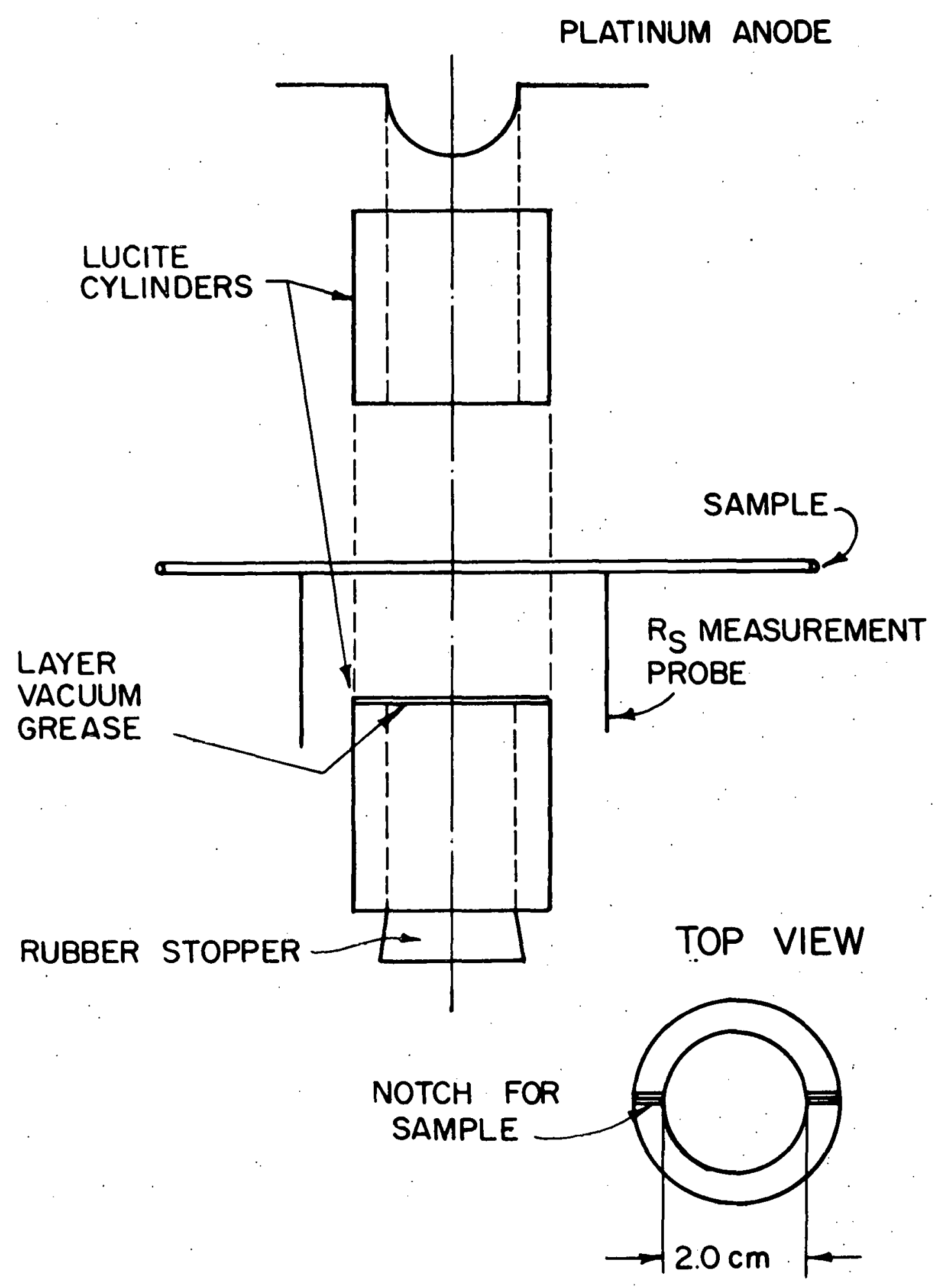

Figure 6. Electrolytic charging jig 
then be calculated knowing the resistance of the specimen before and after introduction of solute and the length of the sample over which the solute proficle extended. Any change in resistance of the hydrogen-free sample was used to correct the measured value of $R_{S}$ for a temperature variation of the oil bath occurring between the initial and final resistance measurements.

The copper leads were then removed from the electromigration specimen and the sample was placed on the sample holder as shown in Figure 5. The measurement of the change in voltage between the knife-edge probes as a function of time was then initiated. It was usually possible to begin the measurement of $\Delta \mathrm{V}_{1-2}, \Delta \mathrm{V}_{2-3}$ and $\mathrm{t}$ within 10 minutes after the electrolytic charging process. Therefore, the solute profile was not significantly modified by diffusion before the determination of $\mathrm{d} \Delta \mathrm{V} / \mathrm{dt}$ was initiated. "In practice, it was possible to measure $\Delta \mathrm{V}_{1-2}$ and $\Delta \mathrm{V}_{2-3}$ in a period of one minute. This rate of measurement was used for the vanadium specimens because of the need to determine $\mathrm{d} \Delta \mathrm{V} / \mathrm{dt}$ in a short time. In niobium and tantalum, measurements were made at three to five minute intervals. Only values of $\Delta V_{1-2}$ and $\Delta V_{2-3}$ which summed to constant value were used in the calculation of $\mathrm{d} \Delta \mathrm{V} / \mathrm{d} t$. This restriction ensures that the sample is at constant temperature. After $d \Delta V / d t$ had been sufficiently well defined, the electromigration current was reversed and $d \Delta V / d t$ was measured for the opposite current 
direction. When $\Delta \mathrm{V}_{1+2}$ and $\Delta \mathrm{V}_{2-3}$ had returned to their initial values, the experiment was terminated.

The sample was immediately cut into three sections. The center segment of the specimen contained all of the hydrogen due to electrolytic charging, while the end sections contained on 1 y a background concentration of hydrogen. The length and weight of each segment was determined after cleaning in acetone and methyl alcohol. The lengths were measured to $\pm 0.5 \mathrm{~mm}$ and the weights to $\pm 0: 0001 \mathrm{gm}$. Using the densities given in Table 2 in addition to the length and weight of the section, the average cross-sectional area of the specimen could be calculated, assuming cylindrical geometry. This method for determination of the average cross-sectional area of the sample appeared to have slightly smaller instrumental uncertainty than a calculation of the area based on a micrometer measurement of the sample diameter.

The amount of hydrogen in each segment was determined by hot vacuum extraction. The hot vacuum extraction apparatus used for all hydrogen analysis is described by Peterson and Fattore (19). The hot vacuum extraction apparatus can accurately measure amounts of hydrogen greater than $1 \times 10^{-6}$ moles with nominal precision of $2 \%$. The average sample size used for analysis was $3 \times 10^{-4}$ moles.

Knowing the number of moles of hydrogen, the cross. sectional area and the charged length of the specimen, it is 
possible to calculate the initial solute concentration. The background hydrogen concentrations were always less than $5 \%$ of the initial solute profile concentration. Probable sources for the background hydrogen concentrations are absorption dur : ing electropolishing or absorption from the mineral oil. Also, volatilization of surface contaminants during hot vacuum extraction would give an indication of a background hydrogen concentration. To ensure that no hydrogen can enter the sample during the electromigration experiment, electropolished specimens were placed in mineral oil for several days. Hot vacuum extraction analysis of these samples indicated virtually no hydrogen was absorbed. Several samples were analyzed for hydrogen content after electropolishing. Again, little, if any hydrogen was found in the specimen.

The resistivity increment due to a unit concentration of solute, $\rho_{S}$, can be calculated knowing $R_{S}$, the cross-sectional area of the sample, A, the electrolytically charged length, $\rho$, and the solute concentration, $c$, from

$$
\rho_{s}=R_{s} \cdot A / c
$$

However,

$$
c=N_{H} \cdot W /(A \cdot l \cdot d)
$$

and

$$
R_{S}=\Delta R / \ell^{\circ}
$$

where $W$ is the atomic weight of the host, $d$ is the density of the host, $\mathrm{N}_{\mathrm{H}}$ is the number of moles of hydrogen in the metal 
and $\Delta \mathrm{R}$ is the increase in resistance of the specimen upon introduction of the solute. Thus, the specific resistivity can be directly calculated from

$$
\rho_{\mathrm{S}}=\Delta \mathrm{R} \cdot \mathrm{A}^{2} \cdot \mathrm{d} /\left(\mathrm{N}_{\mathrm{H}} \cdot \mathrm{W}\right)
$$

From this expression, it can be seen that the specific resistivity is very sensitive to the measurement of the crosssectional area while the measurement of the electrolytically charged length is unimportant. Because of the difficulty in measuring the small cross-sectional area of the electromigration specimens accurately, it is difficult to obtain high precision in the measurement of the specific resistivity of hydrogen isotopes.

\section{Steady-State Electromigration Experiments}

Method of measurement

The presence of a direct electric current in a cylindrical, isothermal electrical conductor can produce a solute flux, J, which may be described at any point along the specimen by

$$
J=c \cdot v-D \cdot \frac{d c}{d x}
$$

$c$ is the solute concentration, $d c / d x$ is the gradient in that concentration, $v$ is the velocity of electromigration and $D$ is the solute diffusivity. Because the velocity of electromigration can be expressed as the product of the electric mobility and electric field, it is possible to cast the expression for the solute flux more generaliy as: 


$$
J=\operatorname{cEU}(c)-D(c) \frac{d c}{d x}
$$

In Equation 20, provision has been made to let the diffusitivy and electric mobility vary with solute concentration.

If the ends of the specimen are impermeable to solute, a steady-state concentration gradient will be established from the balance of the diffusive and electromigration fluxes. When steady-state conditions have been achieved, the net solute flux will be zero at every point along the specimen. Rearranging Equation 20 for the case where $J=0$ yields

$$
\frac{U(c)}{D(c)}=\frac{1}{E} \cdot \frac{1}{c} \cdot \frac{d c}{d x}
$$

Therefore, the ratio of the electric mobility to the solute diffusivity can be determined as a function of solute concentration from the slope of a 1 n $c$ versus $E \cdot x$ plot of the steadystate concentration profile. For the ideal case where $U$ and $D$ are independent of solute concentration, the ln c versus $E \cdot x$ plot of the steady-state solute concentration profile should yield a straight line with slope equal to $U / D$. With the use of Equation 5, U/D can be employed to calculate a value for the effective charged, $Z^{*}$. The steady-state electromigration experiment allows $Z^{*}$ to be calculated without the need to supply diffusion data.

The steady-state technique provides a means by which isotope effects in the $U / D$ ratio can be quite precisely determined. If a single specimen containing hydrogen and deuterium carries 
a direct current until steady-state conditions have been achieved, the concentration profiles of each isotope can be written as

$$
\begin{aligned}
& C_{H}(x) / C_{H}(0)=\exp \left(E x U_{H} / D_{H}\right) \\
& C_{D}(x) / C_{D}(0)=\exp \left(E x U_{D} / D_{D}\right)
\end{aligned}
$$

where $C(0)$ is the concentration of the particular isotope at $x=0$. Equations $22 \mathrm{a}$ and $22 \mathrm{~b}$ assume concentration-independent values for $U$ and $D$, as well as a constant electric field, E. Equations $22 \mathrm{a}$ and $22 \mathrm{~b}$ can be used to predict the ratio of the concentration of an isotope at position $x_{1}$ to the isotope concentration at $x_{2}$.

$$
\begin{aligned}
& \mathrm{C}_{\mathrm{H}}\left(\mathrm{x}_{1}\right) / \mathrm{C}_{\mathrm{H}}\left(\mathrm{x}_{2}\right)=\exp \left(\mathrm{E}\left(\mathrm{x}_{1}-\mathrm{x}_{2}\right) \mathrm{U}_{\mathrm{H}} / \mathrm{D}_{\mathrm{H}}\right) \\
& \mathrm{C}_{\mathrm{D}}\left(\mathrm{x}_{1}\right) / \mathrm{C}_{\mathrm{D}}\left(\mathrm{x}_{2}\right)=\exp \left(\mathrm{E}\left(\mathrm{x}_{1}-\mathrm{x}_{2}\right) \mathrm{U}_{\mathrm{D}} / \mathrm{D}_{\mathrm{D}}\right)
\end{aligned}
$$

After rearrangement of the ratio of Equation 23a to Equation $23 \mathrm{~b}$, the following expression for the isotope effect in the $\mathrm{U} / \mathrm{D}$ ratio can be obtained:

$$
\mathrm{U}_{\mathrm{H}} / \mathrm{D}_{\mathrm{H}}-\mathrm{U}_{\mathrm{D}} / \mathrm{D}_{\mathrm{D}}=\frac{1}{\mathrm{E}\left(\mathrm{x}_{1}-\mathrm{x}_{2}\right)} \ln \left[\frac{\mathrm{C}_{\mathrm{H}}\left(\mathrm{x}_{1}\right) \cdot \mathrm{C}_{\mathrm{D}}\left(\mathrm{x}_{2}\right)}{\mathrm{C}_{\mathrm{H}}\left(\mathrm{x}_{2}\right) \cdot \mathrm{C}_{\mathrm{D}}\left(\mathrm{x}_{1}\right)}\right]
$$

The isotopic concentrations of $x_{1}$ and $x_{2}$ can be related to the fraction of that isotope, $f$, and the total solute concentration, $\mathrm{C}_{\mathrm{T}}$. For example,

$$
\begin{aligned}
& C_{H}\left(x_{1}\right)=C_{T}\left(x_{1}\right) f_{H}\left(x_{1}\right) \\
& C_{D}\left(x_{1}\right)=C_{T}\left(\dot{x}_{1}\right) f_{D}\left(x_{1}\right)
\end{aligned}
$$


Using expressions similar to Equation 25 , Equation 24 can be written as:

$$
U_{H} / D_{H}-U_{D} / D_{D}=\frac{1}{E\left(x_{1}-x_{2}\right)} \ln \left[\frac{f_{H}\left(x_{1}\right) f_{D}\left(x_{2}\right)}{f_{H}\left(x_{2}\right) f_{D}\left(x_{1}\right)}\right]
$$

Thus, knowing only the fraction of hydrogen at two locations along the specimen and the electric field, the isotopic effect in the $U / D$ ratio can be calculated. This method for determination of isotope effects represents a significant improvement for the precision in the measurement of isotope effects compared to results obtainable by two-sample techniques. The number of measurements needed to calculate the isotope effect has been decreased by a factor of two. Also, the substitution of mass spectrographic data for solute concentration analysis represents an improvement due to the greater precision associated with mass spectrography.

The values of $U / D$ for each isotope can be calculated if the total solute concentration is known at $x_{1}$ and $x_{2}$. Rearranging Equations $23 \mathrm{a}$ and $23 \mathrm{~b}$ with the help of Equation 25, the expressions for $U_{H} / D_{H}$ and $U_{D} / D_{D}$ can be written as:

$$
\begin{aligned}
& U_{\mathrm{H}} / \mathrm{D}_{\mathrm{H}}=\frac{1}{\mathrm{E}\left(\mathrm{x}_{1}-\mathrm{x}_{2}\right)} \ln \left[\frac{\mathrm{C}_{\mathrm{T}}\left(\mathrm{x}_{1}\right) \mathrm{f}_{\mathrm{H}}\left(\mathrm{x}_{1}\right)}{\mathrm{C}_{\mathrm{T}}\left(\mathrm{x}_{2}\right) \mathrm{f}_{\mathrm{H}}\left(\mathrm{x}_{2}\right)}\right] \\
& \mathrm{U}_{\mathrm{D}} / \mathrm{D}_{\mathrm{D}}=\frac{1}{\mathrm{E}\left(\mathrm{x}_{1}-\mathrm{x}_{2}\right)} \ln \left[\frac{\mathrm{C}_{\mathrm{T}}\left(\mathrm{x}_{1}\right) \mathrm{f}_{\mathrm{D}}\left(\mathrm{x}_{1}\right)}{\mathrm{C}_{\mathrm{T}}\left(\mathrm{x}_{2}\right) \mathrm{f}_{\mathrm{D}}\left(\mathrm{x}_{2}\right)}\right]
\end{aligned}
$$




\section{Equipment}

The major experimental difficulty associated with the steady-state electromigration experiment is the construction of low electrical resistance connections to the sample with a minimum of current divergence at the junction. Good electrical contact is necessary to prevent excessive power dissipation and the associated temperature rise at the junction. The resulting non-isothermal steady-state concentration profile would be the result of a diffusive, an electromigration and a thermomigration flux. The presence of a thermomigration flux can significantly modify the steady+state electromigration profile making the measurement of the U/D ratio impossible.

For these experiments, $2.3 \mathrm{~mm}(0.090$ inch $)$ copper rods were used to form the junction with the $1.6 \mathrm{~mm}(0.063 \mathrm{inch})$ diameter samples. Holes were drilled longitudinally to a depth of $1.5 \mathrm{~mm}(0.060 \mathrm{inch})$ in one end of each copper rod. The diameter of the hole in the copper rod was the same as the sample diameter to provide a good mechanical. connection. It was sometimes necessary to crimp this junction to ensure good electrical contact. The electrical condition of the junction was checked by measuring the resistance of the copper-samplecopper assembly. A resistance of less than $0.015 \mathrm{ohm}$ for the assembly generally indicated that the junction temperature, as directly measured with a thermocouple, would be elevated 
less than $1^{\circ} \mathrm{C}$ at the current densities used for the steadystate experiments.

The sample-copper rod assemblies were mounted vertically on a bakelite sheet by means of terminals which made electrical contact with the copper rods. The samples were connected in series, with the polarity of adjacent samples being reversed to avoid systematic errors due to long-range temperature gradients. The sample holder, containing approximately ten specimens, was placed in an oil bath to provide a means of maintaining constant sample temperature. Mineral oil was used at $25^{\circ} \mathrm{C}$, while silicone oil was used for the $60^{\circ} \mathrm{C}$ and $120^{\circ} \mathrm{C}$ experiments. The temperature of the oil bath was maintained by a heater which was controlled by a potentiometercontroller system described on page 22. A chrome1-alume1 thermocouple was used as the temperature sensor. 0il temperature regulation, as measured with a mercury thermometer, was better than $2^{\circ} \mathrm{C}$. The oil was continuously stirred to prevent the formation of long-range temperature gradients in the oil bath.

The average electric field was measured by determination of the voltage drop along a known length of the sample. Copper leads of $0.25 \mathrm{~mm}$ ( 0.010 inch) diameter were spot-welded near the ends of the specimen to provide a means of measuring the electric field. The distance between the probes was meas: ured to $\pm 0.5 \mathrm{~mm}$. At the experimental temperatures of $60^{\circ} \mathrm{C}$ and 
$120^{\circ} \mathrm{C}$, it was necessary to extend the electric field probes and the probes for evaluation of the sample-copper junctions outside of the oil bath so that the electric field could be measured and the junctions checked at temperature.

The electromigration current was provided by a D.C. power supply equipped with an automatic "cross-over" feature. The automatic cross-over allowed the power supply to operate in a constant current mode while the load resistance remained below a predetermined value. If the load resistance becomes greater than the preset value, the power supply will switch to a constant voltage mode. This feature was particularly useful for the steady-state electromigration experiments because of the tendency for the resistance of the sample-copper junctions to increase during the course of the experiment. If a junction began to increase in resistance, the switch to a constant voltage mode would prevent the autocatalytic heating of the junction which would characterize the system operating at a constant current. Current densities used for the steady-state electromigration experiments were near 200 amps per $\mathrm{cm}^{2}$.

The hot vacuum extraction equipment described by (19) was used to determine the total hydrogen isotope concentration of sections of the specimen. The hot vacuum extraction system was modified to accept a one liter flask which could be filled with gas extracted from a sample. The gas from these flasks was analyzed for hydrogen and deuterium content by Mr. Gerald 
Flesch, Ames Laboratory.

Experimental procedure

Three niobium-tantalum alloys were prepared at compositions of 25,50 and 75 atomic percent niobium by arc-melting. Rod samples of each of these alloys, in addition to pure niobium, tantalum and vanadium were formed by swaging to $1.6 \mathrm{~mm}$ $(0.063$ inch) diameter. After cutting the specimens to $6 \mathrm{~cm}$ lengths, the samples were electropolished and then annealed by induction heating to $1700^{\circ} \mathrm{C}$ for 20 minutes in an argon atmosphere.

The specimens were then electrolytically charged with one or both hydrogen isotopes as described in Appendix B. The hydrogen isotope concentration was monitored by hot vacuum extraction analysis of a small section of the sample after each electrolytic charging of either hydrogen or deuterium. When the desired hydrogen isotope concentration had been attained, the samples were generally cut to lengths near $4 \mathrm{~cm}$. Several tantalum samples were cut to $3.0 \mathrm{~cm}$ to reduce the time necessary to achieve steady-state conditions. The length of the samples was then measured to $\pm 0.5 \mathrm{~mm}$ and the weights determined to $\pm 0.0001 \mathrm{gm}$. The electric field probes were then spot-welded to the sample and the distance between probes measured to $\pm 0.5 \mathrm{~mm}$. After pressing the copper rods on to the ends of the specimen, all copper surfaces were temporarily covered with an electrically insulating lacquer. The niobium- 
tantalum specimens were then anodized, as described in Appen$\operatorname{dix} B$, to reduce the possibility of solute loss at $60^{\circ} \mathrm{C}$ and $120^{\circ} \mathrm{C}$. The insulating lacquer was removed from the copper surfaces with acetone and the sample-copper rod assemblies were placed on the sample holder and lowered into the oil. The time to achieve steady-state conditions was estimated on the basis of a computer simulation of the experiment using projected values for the electric mobility and diffusivity of hydrogen isotopes in the host metal. It appears as if the time to steady-state conditions is diffusion controlled for experiments where the velocity of migration to diffusivity ratio is less than 0.3 per $\mathrm{cm}$. The relation between the diffusivity, D, sample length, $\ell$, and the time to steady-state, $t_{\text {ss }}$, can be qualitatively described by

$$
\ell=1.4\left(\mathrm{Dt}_{\mathrm{s} s}\right)^{1 / 2} \text {. }
$$

This relation was developed by systematic variation of the input parameters for the computer simulation. A more complete description of the computcr simulation and results of the calculations is given in Appendix C. Times to steady-state for $4 \mathrm{~cm}$ samples ranged from 14 days for niobium at $120^{\circ} \mathrm{C}$ to 100 days for tantalum at $24^{\circ} \mathrm{C}$.

The resistance of the sample-copper junctions was measured periodically during the approach to steady-state and the junctions were repaired if necessary. After steady-state conditions had been achieved, the voltage between the copper 
probes was recorded.

Immediately upon removal from the oil bath, the sample was sectioned into approximately 25 segments. The sample was cut with shears to prevent weight 1oss. The time from removal of the sample until the specimen had been fully sectioned was less than four minutes. A dummy sample was placed in the sample holder to allow current to continue flowing through the remaining specimens in the sample holder. Each segment was cleaned in acetone and methyl alcohol and then weighed to $\pm 0.0001 \mathrm{gm}$. The length of each segment was calculated from the ratio of the segment weight to the total sample weight multiplied by the total sample length. The position with re. spect to the end of the sample of each segment was determined from the sum of the length of the previous segments. The sum of the weight of the segments was compared with the original specimen weight to serve as a check on the conservation of mass of the sample during sectioning and on the accuracy of the measured weight of the segments. In all cases, the difference between the total sample weight and the sum of the weight of the sections was less than $0.3 \%$.

Segments of the specimen were then chosen for mass spectrographic analysis. Segment selection was made on the basis of the need for a minimum amount of gas for mass spectrographic analysis and the condition that the two segments selected be separated by as great a distance as possible. As can be seen from Equation 26, the relative error in the 
measured isotope effect will decrease as the segment separation, $x_{1}-x_{2}$, increases. The segments used for mass spectrographic analysis were not taken from the extreme end of the specimen to avoid possible influences of current divergence and junction heating on the solute distribution. After the mass spectrographic analysis had been performed, the remaining segments were analyzed by hot vacuum extraction to determine the total hydrogen isotope concentration profile.

\section{Electrical Resistivity Measurements}

Electrical resistivity of niobium-tantalum alloys

The electrical resistivity of the three niobium-tantalum alloys was determined by measuring the voltage drop along a given length of a cylindrical specimen at a known current. Specimens used for the resistivity measurement were swaged to $1.6 \mathrm{~mm}(0.063$ inch) diameter and, after electropolishing, were cut to $6 \mathrm{~cm}$ lengths and annealed at $1700^{\circ} \mathrm{C}$ for 20 minutes in an argon atmosphere. The cross-sectional area of each sample was determined, assuming cylindrical geometry, by measuring the weight of a known length of the specimen. Weights were measured to better than $0.5 \%$ and lengths to $1 \%$. The densities needed for the calculation of the cross-sectional area are given in Table 2 .

The sample was placed along a $V$-shaped notch in a bakelite sample holder. Electrical connections were made to both ends of the specimen by means of spring-loaded brass strips. 
which held the sample in the notch. Two tungsten knife-edged rods were mounted on a platform above the sample. This platform was spring supported and the knife-edge voltage probes made electrical contact with the rod specimen when a small weight was placed on the platform. One of the probes was stationary with respect to the platform while the other probe could be driven parallel to the specimen by means of a micrometer. Thus, it was possible to change the spacing between the probes by a known amount through movement of the micrometerdriven probe.

After the resistance of a sample had been measured at a wide probe spacing, the distance between the probes was decreased and the resistance of the specimen was remeasured. Knowing the change in probe spacing and the accompanying change in the measured resistance, the specific resistance of that specimen can be calculated. Multiplying the specific resistance by the cross-sectional area of the sample yields a value for the resistivity of the alloy. The resistivity of two specimens of each alloy was measured to decrease the possibility of a systematic error.

All voltages were measured with a precision potentioneter capable of resolving $1 \times 10^{-6}$ volt. The current was measured by determining the voltage drop across a standard resistance which was placed in series with the sample. Current densities used for the measurements were near $15 \mathrm{amps} / \mathrm{cm}^{2}$. The resistiv- 
ity of all samples was measured within a period of less than one hour over which time the ambient temperature varied less than $1^{\circ} \mathrm{C}$ from $22^{\circ} \mathrm{C}$.

Hydrogen isotope electrical resistivity

The resistivity increase due to a unit concentration of hydrogen and deuterium in the niobium-tantalum alloys was measured with techniques identical to those used for the electric mobility measurements. Sample preparation and dimensions, as well as procedures for determination of the solute resistivity can be found on page 37 .

Hydrogen Diffusivity Measurement

From a theoretical standpoint, there is much interest in knowing how transport properties of hydrogen vary as the niobium-tantalum alloy solid solution range is traversed. The ratio of the electric mobility to hydrogen diffusivity was measured with steady-state methods. The variation of the diffusion coefficient of hydrogen as a function of niobium-tantalum alloy composition was unknown. To provide insight into this problem, the diffusivity of hydrogen at $23^{\circ} \mathrm{C}$ in the three niobium-tantalum alloys used for the steady-state electromigration experiments was measured.

The determination of hydrogen diffusivity was made by examination of solute concentration-distance profiles obtained by diffusion of a nearly infinite source of hydrogen through a 
constant concentration boundary into an infinite rod. When the diffusion time and the hydrogen concentration as a function of distance is known, it is possible to calculate the diffusion coefficient of hydrogen as a function of hydrogen concentration using the Boltzmann-Matano Solution (20)

$$
D\left(c^{\prime}\right)=-\frac{1}{2 t}\left(\frac{d x}{d c}\right) c_{c^{\prime}} \int_{0}^{c^{\prime}} x d c
$$

The definition of $x=0$ in Equation 29 can be taken as the location of the constant hydrogen concentration boundary. It may be noticed that the definition of $x=0$ for the case of interstitial diffusion differs somewhat from the definition of $x=0$ for substitutional diffusion. The separate definitions of the $x=0$ plane arise from the different conservation of mass principles which must be applied to interstitial and substitutional diffusion.

A major difficulty encountered with the practical application of Boltzmann-Matano solution is the need to have solute concentration profiles of very high accuracy in order that the concentration gradient can be determined with adequate accuracy. Fortunately, hot vacuum extraction of hydrogen in the Group VB metals can provide precise hydrogen concentration data from a relatively small specimen. Therefore, the concentration of hydrogen could be determined at many points along the solute profile with great enough precision to make application of the Boltzmann-Matano solution possible. 
Samples of niobium, tantalum and the three intermediate alloys were prepared by swaging to $1.6 \mathrm{~mm}(0.063$ inch) diameter and then cutting to $6 \mathrm{~cm}$ lengths. All samples were annealed at $1700^{\circ} \mathrm{C}$ for 20 minutes in an argon atmosphere. The weight and length of each sample was then measured. After electropolishing the entire specimen, approximately $1 \mathrm{~cm}$ of the sample was immersed in a $20 \% \mathrm{H}_{2} \mathrm{SO}_{4}-\mathrm{H}_{2} \mathrm{O}$ solution at $60^{\circ} \mathrm{C}$ and was electrolytically charged for 10 minutes at a current density of $20 \mathrm{amps} / \mathrm{cm}^{2}$.

The electrolytic introduction of a high solute concentration in a $1 \mathrm{~cm}$ length of the sample created a diffusion couple which satisfies the boundary conditions necessary for the application of Equation 29. The electrolytically charged region was usually found to have a hydrogen to metal ratio of greater than 0.4 . This concentration greatly exceeds the solubility limit for hydrogen in the metal and as a result, a two phase region consisting of alpha solid solution and hydride was formed. The hydrogen concentration at the boundary between the two-phase region and the hydrogen-free section of the sample was fixed at the solubility limit for hydrogen in the alpha phase. Thus, the two-phase region acts as a source of hydrogen which fixes a constant concentration of hydrogen at a specific location in the specimen. From this constant concentration boundary, hydrogen diffuses into the solute-free region of the specimen. 
Technically speaking, the boundary between the two-phase and single phase regions of the sample does not remain stationary with respect to the ends of the specimen. Because of the finite hydrogen concentration, the extent of the two-phase region must diminish as hydrogen diffuses into the singlephase region. Because the hydrogen concentration of the twophase region ranges from 4 to 25 times the interface concentration and the time of the diffusion experiment is not excessive, the total distance over which the interface moves will be sma11.

The time for which hydrogen was allowed to diffuse was determined from the requirement that the diffusing hydrogen not reach the end of the specimen. In practice, this time was near one day for niobium and three days for tantalum at $23^{\circ} \mathrm{C}$ and for maximum diffusion distances of $4 \mathrm{~cm}$.

After the hydrogen had been allowed to diffuse for a measured amount of time, the sample was sheared into approximately 30 segments. Each segment was cleaned with acetone and methyl alcohol and then weighed. The length of each segment was determined from the ratio of the segment weight to total sample weight multiplied by the original sample length. The position of each segment with respect to the end of the rod was determined from the sum of the length of the previous segments. The hydrogen concentration of each segment was then determined by hot vacuum extraction. 
With the aid of solubility data (9), and extrapolation of the concentration-distance profile, the location of the $x=0$ interface could be established. The uncertainty in the location of the $x=0$ plane was usually small because of the small segment size $(2 \mathrm{~mm})$ and the precipitous drop in hydrogen concentration at the $x=0$ interface. The Boltzmann-Matano solution can then be used to calculate the diffusivity of hydrogen as a function of solute concentration.

This technique is most successful for niobium and tantalum near $23^{\circ} \mathrm{C}$. Because of the sharply rising solubility limit for hydrogen as the temperature is increased, it becomes impossible to produce a two-phase region with a hydrogen concentration which is much greater than the solubility limit. When this occurs, there can be significant movement of the $x=0$ interface during the time when hydrogen is diffusing into the single phase region. When significant movement of the $x=0$ interface occurs, application of Equation 29 is invalid. 
RESULTS

Sample Characterization

\section{Interstitial impurities}

Much discussion has centered on the effect of other interstitial elements on the observed behavior of hydrogen. Most often, an attractive interaction between hydrogen and the other interstitial elements, carbon, nitrogen and oxygen, is thought to exist. This phenomenon is generally called "Trap-. ping". Although the understanding of trapping is far from complete, several studies on niobium have indicated that a single hydrogen atom can be bound to an oxygen or nitrogen atom. The binding energy for trapping was observed to be similar in magnitude to the activation energy for hydrogen diffusion in niobium $(21,22)$. Thus, it appears as if trapping by oxygen and nitrogen could significantly affect the measurement of hydrogen transport properties.

To be certain that the measured transport parameters are those intrinsic to the metal-hydrogen system under study, it is necessary to know the concentration of carbon, nitrogen and oxygen in the specimens used for the transport measurement. For determination of oxygen and nitrogen levels, vacuum fusion analysis was used. Carbon combustion was employed to determine carbon content. The results of these analyses are given in Table 1. The impurity levels listed in Table 1 are average values from several concentration measurements. Samples selec- 
ted for these analyses had been annealed. Several samples were taken from specimens after they had been used for actual electromigration experiments.

Normal hydrogen concentrations used for the electromigration and diffusion experiments generally exceeded total impurity content by more than an order of magnitude. Therefore, it would appear as if trapping of hydrogen by carbon, nitrogen and oxygen should not affect the measurement of hydrogen transport properties significantly.

Table 1. Average carbon, nitrogen and oxygen content of the vanadium, niobium and tantalum specimens in atomic percent

\begin{tabular}{cccc}
\hline & $\mathrm{V}$ & $\mathrm{Nb}$ & $\ldots . \mathrm{T} \ldots \ldots .04$ \\
\hline $\mathrm{N}$ & 0.02 & 0.10 & 0.04 \\
$\mathrm{C}$ & 0.01 & 0.01 & 0.02 \\
\hline
\end{tabular}

\section{Alloy composition}

The niobium-tantalum alloys were prepared by arc melting measured amounts of the pure metals together. Weight loss during arc melting was small. Assuming that all weight loss was due to vaporization of niobium, the maximum deviation from the 25,50 and 75 atomic percent alloy compositions is 0.9 atomic percent. Density and atomic weight

In calculation of hydrogen concentration and crosssectional area from specimen weight data, it is necessary 
to supply values for the density and atomic weight of the metal under study. For pure metals, these data are readily available, however, densities and atomic weights of alloys are not commonly tabulated. Fortunately, niobium-tantalum alloys form an ideal system from which densities and atomic weights can be easily calculated. The lattice parameter of pure niobium and tantalum differ by on $1 y 0.06 \%$ and in the intermediate solid solution range, the lattice parameter is a linear function of alloy composition (23). Thus, the densities and atomic weights for niobium-tantalum alloys can be calculated from averages of pure metal properties weighted according to alloy composition. The values of density and atomic weight for all metals used in this research are given in Table 2 .

\section{Electric Mobility Measurements}

The migration of hydrogen isotopes in response to an applied electric field was found to be directed toward the cathode in vanadium, niobium and tantalum. The observation of a positive effective valence for hydrogen isotopes is in agreement with other electromigration measurements on these systems $(2,13)$.

The electric mobility of hydrogen and deuterium in vanadium and niobium is shown as a function of solute concentration in Figure 7. There appears to be no strong concentration dependence for the electric mobility of hydrogen isotopes 
Table 2. Density and atomic weight of vanadium, niobium, tantalum and niobiumtantalum alloys near $24^{\circ} \mathrm{C}$.

\begin{tabular}{lcccccc}
\hline & $\mathrm{V}$ & $\mathrm{Nb}$ & $75 \mathrm{Nb}-25 \mathrm{Ta}$ & $50 \mathrm{Nb}-50 \mathrm{Ta}$ & $25 \mathrm{Nb}-75 \mathrm{Ta}$ & $\mathrm{Ta}$ \\
\hline $\begin{array}{l}\text { Density } \\
\left(\mathrm{gm} / \mathrm{cm}^{3}\right)\end{array}$ & 6.0 & 8.57 & 10.6 & 12.6 & 14.6 & 16.6 \\
$\begin{array}{l}\text { Atomic weight } \\
(\mathrm{gm} / \text { mole) }\end{array}$ & 50.95 & 92.91 & 114.9 & 136.9 & 158.9 & 180.95 \\
\hline
\end{tabular}




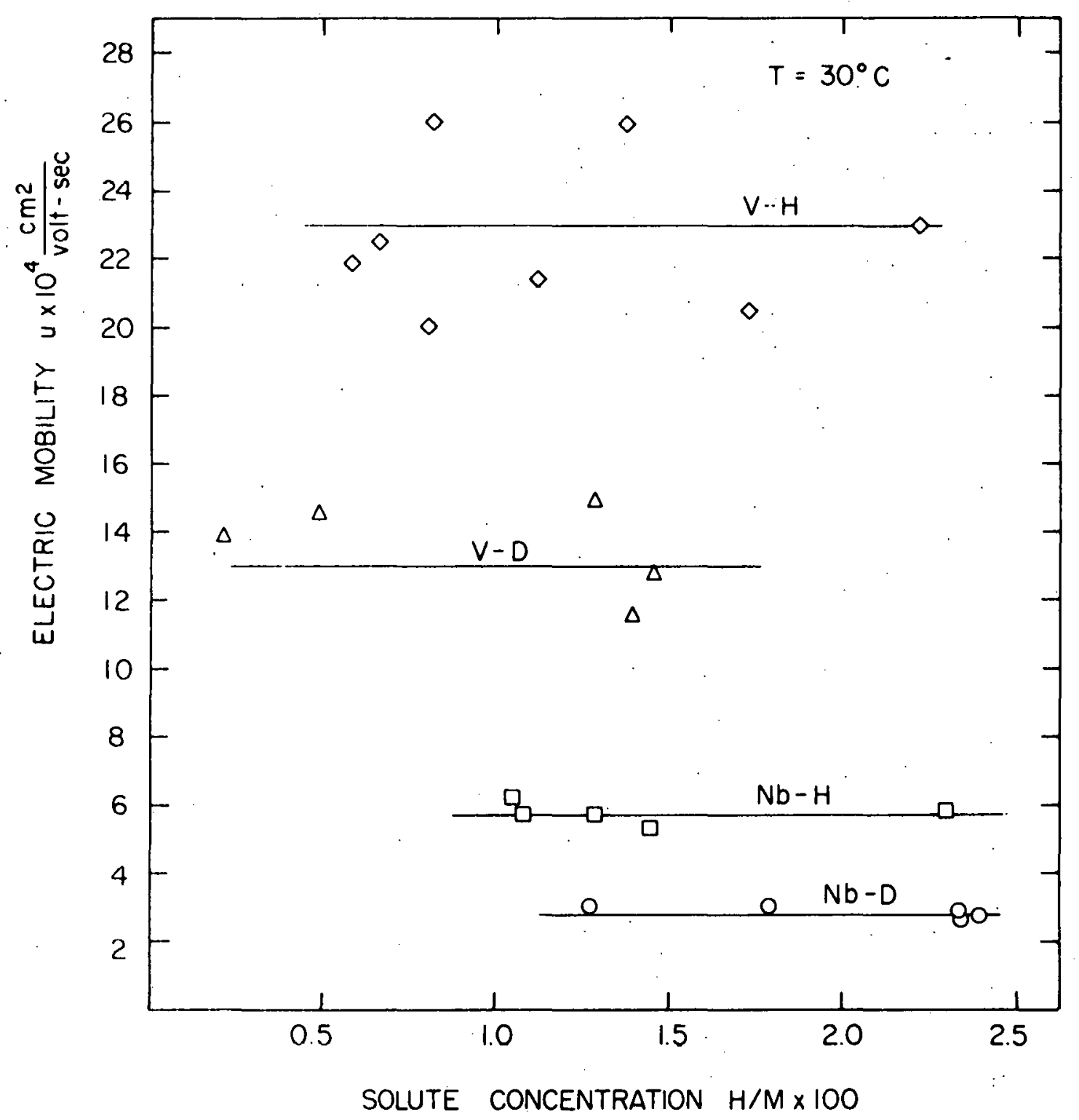

Figure 7. Electric mobility of hydrogen and deuterium versus solute concentration in niobium and vanadium at $30^{\circ} \mathrm{C}$ 
in vanadium and niobium at $30^{\circ} \mathrm{C}$.

Table 3 presents the average values for the elastic mobility of hydrogen isotopes in niobium and vanadium. Although the magnitude for the electric mobility of hydrogen isotopes in vanadium is a factor of four greater than in niobium, the isotopic ratio of the electric mobilities is nearly equal for both metals. In classical terms, the electric mobility of hydrogen would be expected to be greater than that for deuterium by a factor of 1.41. It appears from the data in Table 3 that the isotopic ratio exceeds the classically expected value in both vanadium and niobium. The electric mobility data obtained at $600^{\circ} \mathrm{C}$ with gas flow techniques by Mareche, Rat and Herold (13) show isotopic ratios of 1.3 and 1.5 for the electric mobility in vanadium and niobium, respectively.

It is, perhaps, noteworthy that the diffusivity of hydrogen isotopes also appears to exhibit classical behavior at elevated temperatures. For example, the isotopic ratio for diffusion of hydrogen isotopes in vanadium decreases from 3:0 at $30^{\circ} \mathrm{C}$ to the classical value of 1.4 at $600^{\circ} \mathrm{C}(10)$. The existence of non-classical isotope effects in the diffusivity and electric mobility at low temperatures may be a reflection of quantum effects which influence the transport behavior of hydrogen isotopes.

Also listed in Table. 3 are the hydrogen isotope diffusi- 
Table 3. Electric mobility, diffusivity, effective charge and solute resistivity for $H$ and $D$ in $N b$ and $V$. at $3.0^{\circ} \mathrm{C}$

\begin{tabular}{lcccccc}
\hline & $\mathrm{H}-\mathrm{V}$ & $\mathrm{D}-\mathrm{V}$ & $\frac{\mathrm{H}-\mathrm{V}}{\mathrm{D}-\mathrm{V}}$ & $\mathrm{H}-\mathrm{Nb}$ & $\mathrm{D}-\mathrm{Nb}$ & $\frac{\mathrm{H}-\mathrm{Nb}}{\mathrm{D}-\mathrm{Nb}}$ \\
\hline $\mathrm{Ux} 10^{4} \mathrm{~cm}^{2} / \mathrm{volt-sec}$ & $23 \pm 1$ & $13 \pm 1$ & 1.8 & $5.7 \pm 0.1$ & $2.8 \pm 0.1$ & 2.0 \\
$\mathrm{Dx} 10^{6} \mathrm{~cm}^{2} / \mathrm{sec}$ & 52 & 17 & 3.1 & 8.6 & 3.9 & 2.2 \\
$\mathrm{Z}^{*}=\mathrm{UkT/(De)}$ & +1.1 & +2.1 & 0.52 & +1.7 & +1.9 & 0.89 \\
$\rho_{\mathrm{s}} \times 10^{6} \frac{\mathrm{ohm}-\mathrm{cm}}{(\mathrm{H} / \mathrm{m}) 100}$ & $0.58+0.02$ & $.90 \pm 0.03$ & 1.1 & $0.61 \pm 0.01$ & $0.55 \pm 0.01$ & 1.1 \\
\hline
\end{tabular}


vities at $30^{\circ} \mathrm{C}$ calculated from the data of (10). It should be noted that the diffusivities appearing in Table 3 have been extrapolated to infinite solute dilution. Thus, these diffusivities should represent the intrinsic diffusivity of hydrogen isotopes, $D^{*}$. The intrinsic diffusivity is related to the concentration-dependent diffusivity, $D(c)$, by (24)

$$
D(c)=D^{*}(1+d \ln \gamma / d \ln c) \text {. }
$$

Substituting Equation 30 into the expression used for calculation of the effective valence, Equation 5, yields

$$
Z^{*}=\frac{U k T}{D^{*} \mathrm{e}}
$$

Thus, knowing $D^{*}$ eliminates the need for activity coefficient data in the calculation of the effective valence from electric mobility data. The calculated effective valence for hydrogen and deuterium in niobium and vanadium is listed in Table 3 . It is interesting to note that the isotopic ratio for the solute diffusivity is less classical than the ratio of the electric mobilities. The net result is that the calculated effective valence for deuterium is larger than that for hydrogen because $Z^{*}$ involves the ratio, U/D.

The experimental precision in the electric mobility data, as evidenced by Figure 7, appears to be less than would be estimated from the instrumental uncertainties involved in the measurement of the electromigration velocity. While the electromigration current can be determined with negligible uncer- 
tainty, a possible error of near $3 \%$ may exist in the measurement of the solute resistance, $R_{s}$. The slope of the voltage versus time data is also we11-defined, as can be seen in some experimental data shown in Figure 8. The total instrumental uncertainty for the measurement would be estimated as less than $10 \%$.

The probable cause for the lack of precision in the experimental electric mobility data is the presence of solute concentration gradients in the solute concentration profile. The assignment of the value of $R_{s}$ averaged over the entire solute distribution to the small portion of the profile responsible for the measured resistance change due to electromigration will introduce random errors if the solute profile contains concentration gradients. Although solute nonuniformity near the center of the concentration profile can be detected by reversal of the electromigration current, it is impossible to detect concentration gradients which occur near the ends of the solute profile.

The behavior of the electric mobility of hydrogen isotopes in tantalum appears to be markedly different than for the results in vanadium and niobium. Initial migration velocity measurements for hydrogen in tantalum were done, unintentionally, at rather low solute concentrations $(<1.5$ atomic percent) due to the low efficiency of electrolytic introduction of hydrogen into tantalum. No solute motion could be 


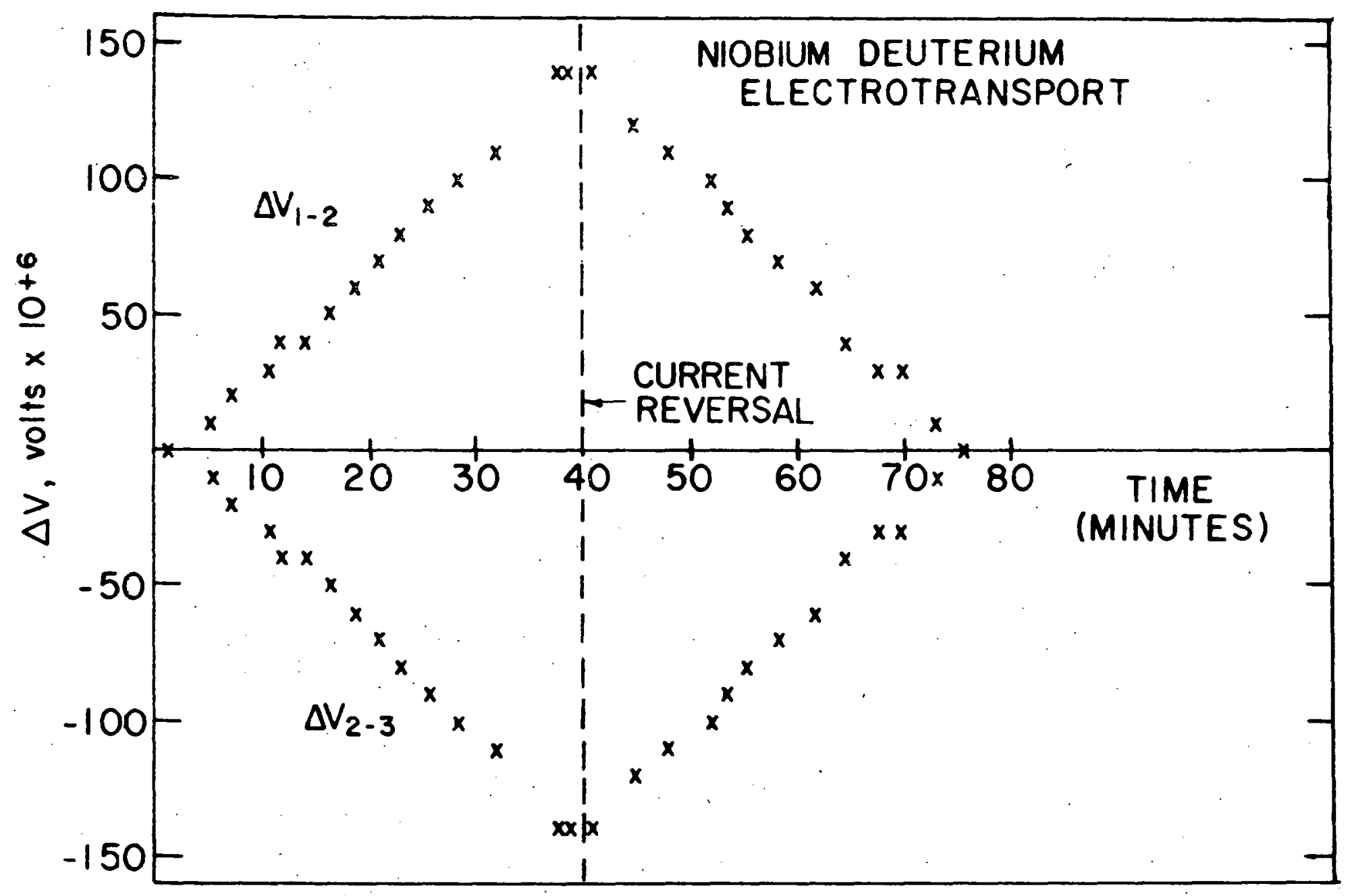

Figure 8. Sample voltage versus time data for electric mobility measurement of deuterium in niobium at $30^{\circ} \mathrm{C}$ 
detected in either current direction in times which were an order of magnitude longer than should have been necessary to observe resistance changes. These results were initially ascribed to very non-uniform solute profiles caused by the inefficient electrolytic charging. The electric mobility measurements were then discontinued in favor of the steadystate technique for observation of electromigration.

The steady-state experiments suggested that the electric mobility of hydrogen isotopes markedly decreases for hydrogen isotope concentrations below three atomic percent. This result prompted direct electric mobility measurements at solute concentrations higher than had previously been used. At higher solute concentrations, measurable solute movement was observed and agreed well with results of the steady-state experiments. Thus, it appears as if the extremely low migration velocity indicated by the electric mobility measurements at low solute concentrations was not an erroneous result. Further discussion of this concentration dependence will be deferred to the results of the steady-state technique.

\section{Steady-State Electromigration Measurements}

Thermodynamic correction factor

Calculation of $Z^{*}$ from steady-state electromigration experiments requires a value of $U / D$ obtained from the slope of the solute concentration profile and a knowledge of the thermodynamic correction factor, $d$ in $\mathrm{r} / \mathrm{d}$ in $\mathrm{c}$. Equilibrium 
pressure-composition experimenta are generally used to provide data for calculation of the thermodynamic correction factor for hydrogen dissolved in metals. As seen from Equation 7, the thermodynamic correction factor is related to the rate of change of equilibrium hydrogen pressure as the hydrogen concentration is varied. If the square root of the equilibrium pressure is directly proportional to the solute concentration, then the thermodynamic correction factor is equal to zero. Pressure-composition experiments are generally restricted to high temperatures in order that equilibrium between hydrogen in the gas and solid phases be readily attained. It is necessary to extrapolate thermodynamic data nearly $500^{\circ} \mathrm{C}$ to obtain a thermodynamic correction factor which can be applied at $24^{\circ} \mathrm{C}$ for calculation of $z^{*}$. Because the determination of a low temperature value for the thermodynamic correction factor involves a long extrapolation of the slope of pressurecomposition data, it might be expected that the calculated thermodynamic factor will contain significant uncertainty.

One method by which the validity of the calculated thermodynamic factor can be verified is by calculation of the concentration-independent, or intrinsic diffusivity, from experimental diffusion data. As can be seen from Equation 30, division of the experimentally measured diffusivity by $1+\mathrm{d} \ln \mathrm{r} / \mathrm{d}$ ln $\mathrm{c}$ should yield a concentration-independent diffusivity. As will be discussed in connection with the diffusivity measurements, 
an extrapolated thermodynamic correction factor was not able to remove the concentration dependence from the experimentally measured diffusivity in niobium and tantalum.

Because the combination of the experimentally determined hydrogen diffusivity and thermodynamic correction factor does not produce a concentration-independent diffusivity, the absolute mobility defined by Equation 1, is also dependent on solute concentration. The concept of absolute mobility was introduced as a fundamental property of all transport mechanisms, independent of solute concentration. Because the calculated absolute mobility does not meet the basic requirements of its definition, the application of the calculated absolute mobility in discussion of other transport processes may not be useful. "The absolute mobility does enter into the calculation of $z^{*}$, as can be seen from Equation 4. To avoid possible confusion due to the lack of knowledge concerning the thermodynamic correction factor, experimental data will generally be presented in terms of the measured quantity, U/D. If use of the effective valence becomes necessary, $Z^{*}$ will be calculated assuming the thermodynamic correction factor to be zero. Calculation of $\mathrm{U} / \mathrm{D}$

As was discussed in connection with Equation 21 , U/D can be determined at each position along a specimen from the slope of a ln $c$ versus $E \cdot x$ plot of the steady-state solute concentration profile. If this plot yields a straight line, $U / D$ is . 
independent of concentration for the range of solute concentration existing in the specimen.

If hydrogen and deuterium are present in a single specimen, each isotope will establish a steady-state concentration profile characteristic of its own U/D ratio. Experimentally, it has been found that the isotope effect in U/D is small for hydrogen isotopes in the Group VB metals. Therefore, the concentration profile of each isotope and the total hydrogen isotope concentration profile can be characterized by a single value of $U / D$. Figure 9 shows $1 \mathrm{n}\left(\mathrm{c}_{\mathrm{H}}+\mathrm{c}_{\mathrm{D}}\right)$ versus $\mathrm{E} \cdot \mathrm{x}$ data for five niobium-tantalum systems at $60^{\circ} \mathrm{C}$. There appears to be no significant deviation from linearity for each specimen. Thus, hydrogen and deuterium can be assigned nearly identical values of $U / D$ which are independent of concentration for the range of solute concentrations existing in the specimen.

In order that $U / D$ be calculated for each isotope using Equation 27 , it is necessary to know the total hydrogen isotope concentration at the locations where the fraction of hydrogen has been determined. Because of the exponential behavior of the total solute profile, the total hydrogen isotope concentration at any position along the specimen can be determined from the parameters obtained from a least-squares treatment of the $\ln \left(c_{H}+c_{D}\right)$ versus $E \cdot x$ data. The uncertainty associated with the solute concentration at a specific location can be considerably reduced by the analytic method rather 


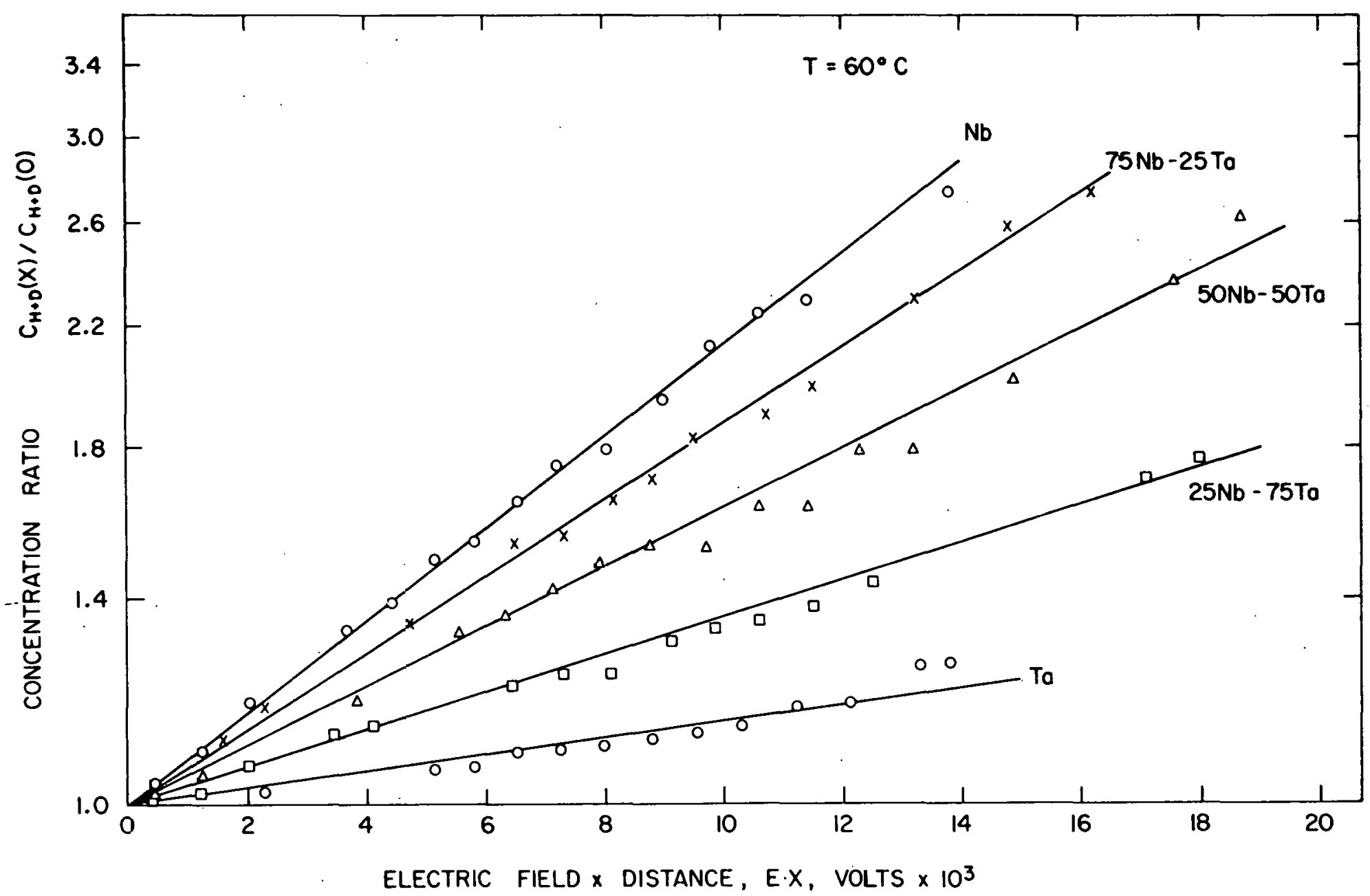

Figure 9. In $\mathrm{c}_{\mathrm{H}+\mathrm{D}}(\mathrm{x}) / \mathrm{c}_{\mathrm{H}+\mathrm{D}}(0)$ versus $\mathrm{E} \cdot \mathrm{x}$ for niobium-tantalum alloys at $60^{\circ} \mathrm{C}$ 
than relying on a single concentration measurement.

The uncertainty in $U / D$ and the isotope effect in this quantity comes from four sources. The determination of the electric field requires the measurement of the distance between voltage probes. The instrumental uncertainty in this measurement is near $3 \%$. The determination of the fraction of each isotope at specific locations in the specimen can be made with a precision of \pm 0.001 in the fraction of the isotope measured. The distance between the locations where the fraction of hydrogen has been measured can be determined with a precision of $2 \%$ from the segment weights and the length and weight of the original specimen. The uncertainty in the total hydrogen isotope concentration at any position along the specimen is near $3 \%$.

An error analysis based on the expression used to calculate $U_{H} / D_{H}-U_{D} / D_{D}$, Equation 26 , shows that the magnitude of the error in this quantity is independent of the magnitude of $\mathrm{U}_{\mathrm{H}} / \mathrm{D}_{\mathrm{H}}-\mathrm{U}_{\mathrm{D}} / \mathrm{D}_{\mathrm{D}}$. Thus, the relative error in the isotope effect decreases when the measured isotope effect becomes larger. The average minimum detectable magnitude of $U_{H} / D_{H}-U_{D} / D_{D}$ for the experimentally used electric fields and specimen lengths is 0.5 volt ${ }^{-1}$.

An error analysis for the expression used to calculate U/D for each isotope, Equation 27, shows that the relative error in $U / D$ decreases as the gradient in the solute concen- 
tration profile is increased. The nominal uncertainty in U/D is less than $10 \%$ except for the very low values of $U / D$ obtained for low solute concentrations in tantalum. For values of $\mathrm{U} / \mathrm{D}$ less than 10 volts $^{-1}$, the estimated error may reach $50 \%$. $\underline{\text { Vanadium }}$

A specimen containing only hydrogen was first used to verify the exponential dependence of the steady-state concentration profile. This was done to check the assumption of exponential profile shape made in the derivation of the relations from which $U / D$ and isotope effects in this ratio are calculated. The exponential character of the hydrogen concentration profile was verified within experimental error. Two specimens containing hydrogen and deuterium were then used to measure $U / D$ for both isotopes at $24^{\circ} \mathrm{C}$. The average $U / D$ ratio for hydrogen was measured to be $+54.0 \pm 0.2$ volt ${ }^{-1}$ while U/D for deuterium in vanadium was found to be $+57.7 \pm 0.1 \operatorname{volt}^{-1}$. The effective valence for hydrogen and deuterium in vanadium can be calculated to be +1.41 and +1.53 , respectively. The hydrogen-to-deuterium ratio of these quantities is 0.92 . The effective valence, reported in Table 3 , from the electric mobility measurements is +1.2 for hydrogen and +2.0 for deuterium in vanadium. Because of the greater precision associated with $Z^{*}$ as measured by steady-state methods, it is believed that the steady-state results are more reliable. This is especially true with regard to the magnitude of the 
isotope effect.

Niobium-tantalum alloys

The experimentally measured U/D ratio for hydrogen isotopes in five niobium-tantalum systems at $24^{\circ} \mathrm{C}, 60^{\circ} \mathrm{C}$ and $120^{\circ} \mathrm{C}$ is shown in Figures 10,11 and 12. The largest isotope effect in $U / D$ was observed in niobium at $24^{\circ} \mathrm{C}$. The $U / D$ ratio of deuterium exceeded that for hydrogen by $14 \%$. In every other instance, isotope effects in $U / D$ were less than $5 \%$ of the magnitude of U/D. Because of the small isotope effect in $U / D$ observed in the niobium-tantalum systems, a single point has been used to represent the average $U / D$ ratio for hydrogen and deuterium in Figures 10, 11 and 12. Because of the strong concentration dependence of $U / D$ for hydrogen isotopes in tantalum, only samples containing concentrations greater than $4.0(\mathrm{H} / \mathrm{M}) \times 100$ have been used to obtain the average $U / D$ ratio for tantalum in Figures 10,11 and 12 . The U/D ratio appears to become relatively independent of hydrogen isotope concentration above $4.0(\mathrm{H} / \mathrm{M}) \times 100$ in tantalum.

The temperature dependence of $Z^{*}$ is shown in Figure 13. Within experimental error, there appears to be no significant temperature dependence in the effective valence from $24^{\circ} \mathrm{C}$ to $120^{\circ} \mathrm{C}$. Although the temperature range of the experiment was not large, the average temperature-induced resistivity change for the niobium-tantalum systems was $25 \%$.

The U/D ratio, obtained from steady-state and electric 


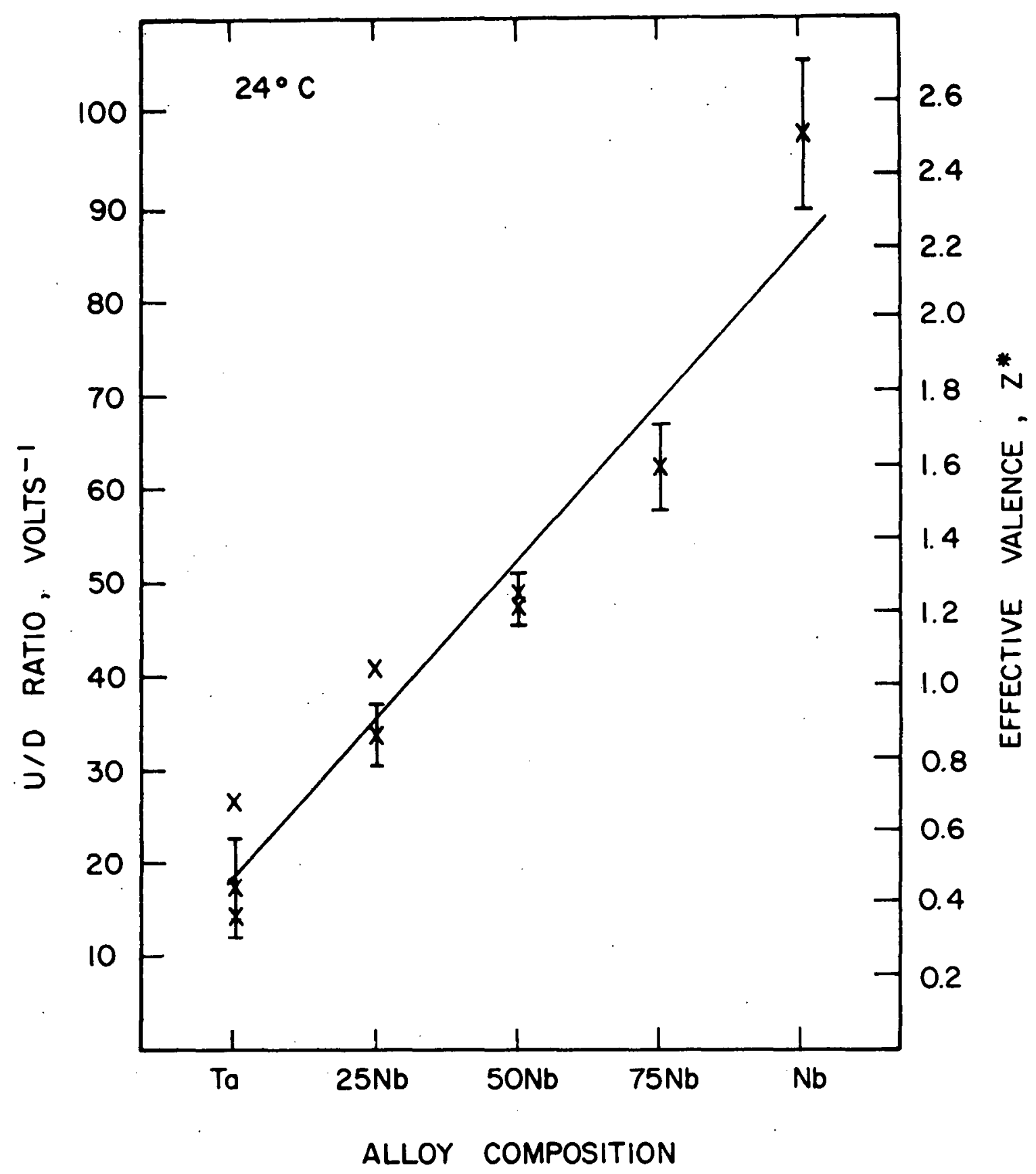

Figure 10. Average U/D ratio for hydrogen and deuterium as a $24^{\circ} \mathrm{C}$ fion of niobium-tantalum alloy composition at 


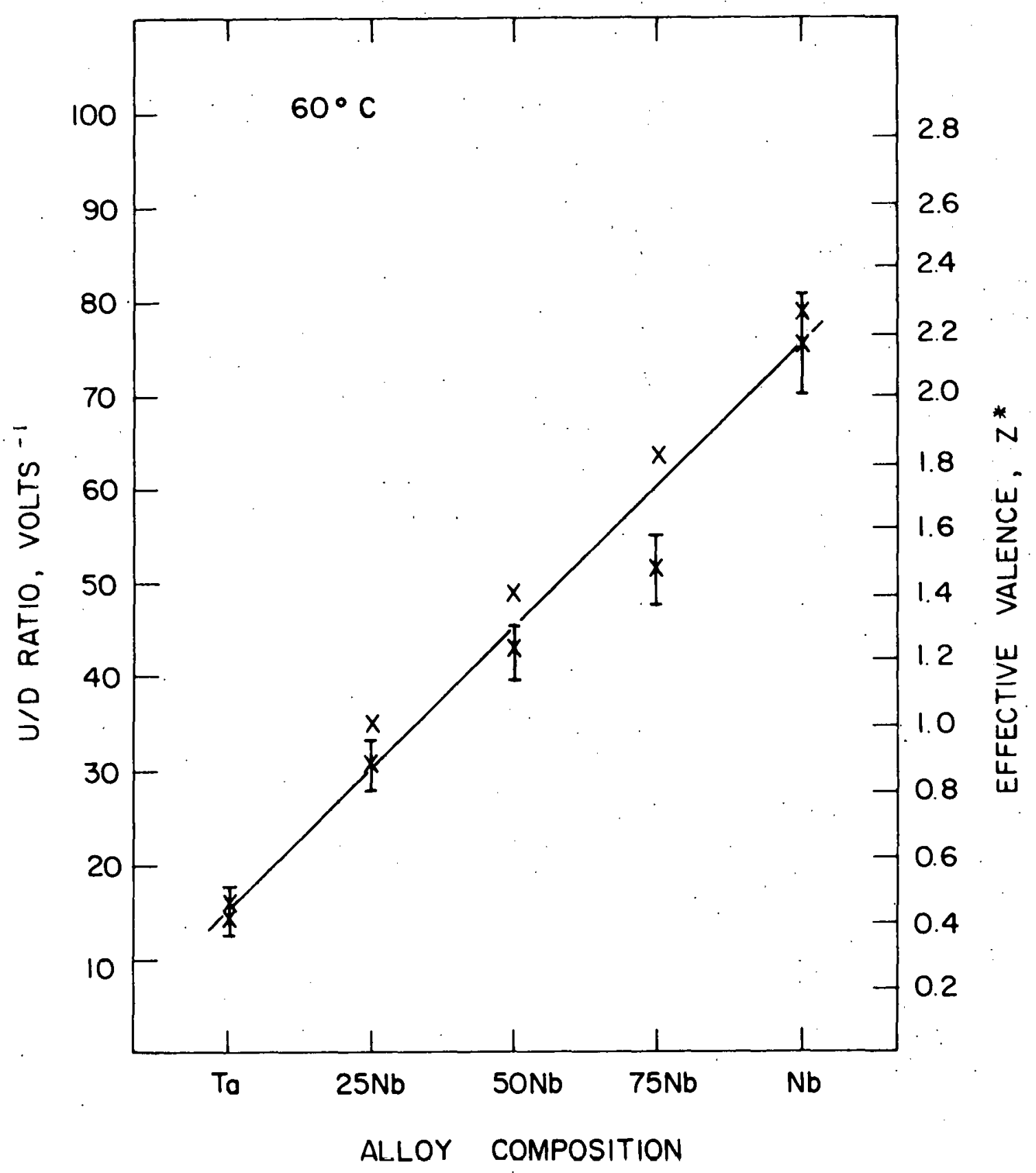

Figure 11. Average U/D ratio for hydrogen and deuterium as a function of niobium-tantalum alloy composition at $60^{\circ} \mathrm{C}$ 


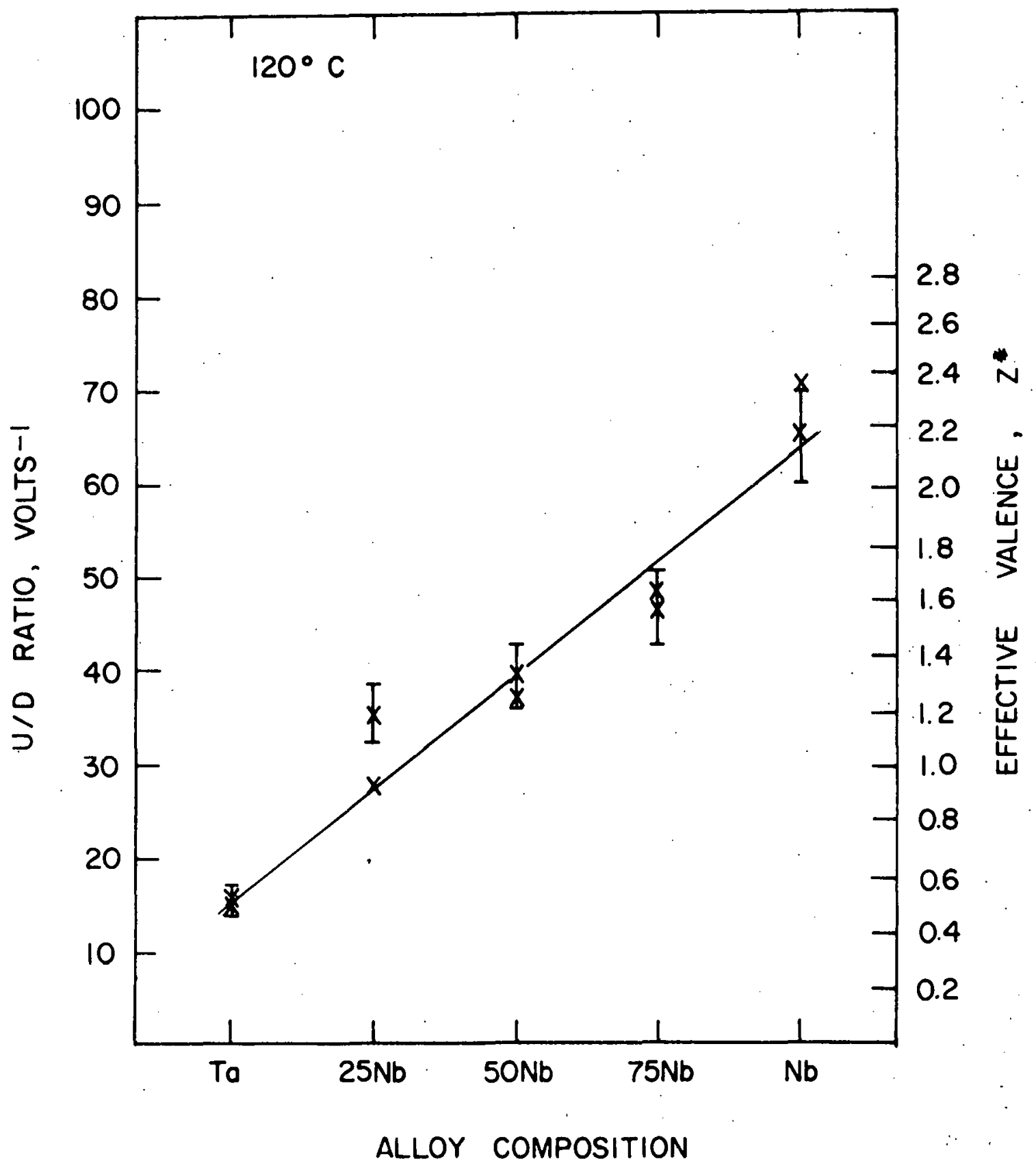

Figure 12. Average $U / D$ ratio for hydrogen and deuterium as a function of niobium-tantalum alloy composition at $120^{\circ} \mathrm{C}$ 


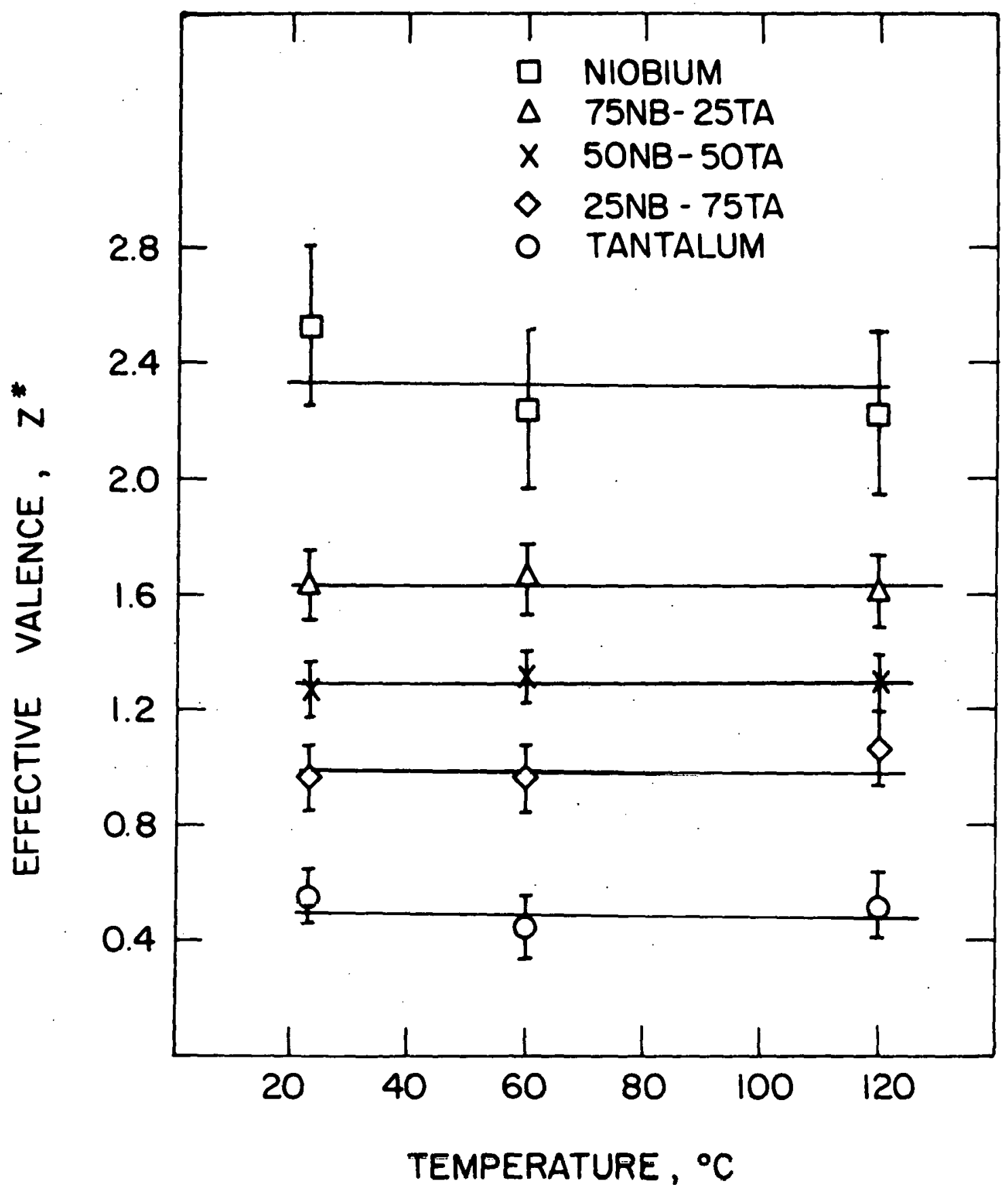

Figure 13. Average effective valence of hydrogen and deuterIum as a function of temperature for niobiumtantalum alloys 
mobility measurements, for hydrogen isotopes in tantalum as a function of total hydrogen isotope concentration is shown in Figure 14. Although the relative uncertainty in $U / D$ becomes quite large, there appears to be a reversal of electromigration direction at solute concentrations below $1.5(\mathrm{H} / \mathrm{M}) \times 100$. The form of the concentration dependence, including the reversal of migration direction, is in agreement with the electric mobility measurements of Ivashina, Nemchenko and Charnetskiy (25). They reported a change in sign for the migration direction of hydrogen in tantalum at a concentration of $1.3(\mathrm{H} / \mathrm{M}) \times 100$. However, the absolute magnitude of the electric mobility observed by (25) appears to be nearly two orders of magnitude greater than the electric mobilities calculated from the data in Figure 14.

Because of the small values of $U / D$ and $E$, the concentration range spanned by each specimen is small. Thus, it was not possible to detect the concentration dependence of $U / D$ directly from the $\ln \left(c_{H}+c_{D}\right)$ versus $E \cdot x$ plot of the steadystate concentration profile for a single specimen.

Electric mobility and steady-state experiments in niobium and vanadium show no concentration dependence in $U / D$ for solute concentrations greater than $0.7(\mathrm{H} / \mathrm{M}) \times 100$. The extent to which the concentration dependence in $U / D$ occurs in the niobium-tantalum alloys is unknown because only data for high solute concentrations was obtained. The effect of temperature 


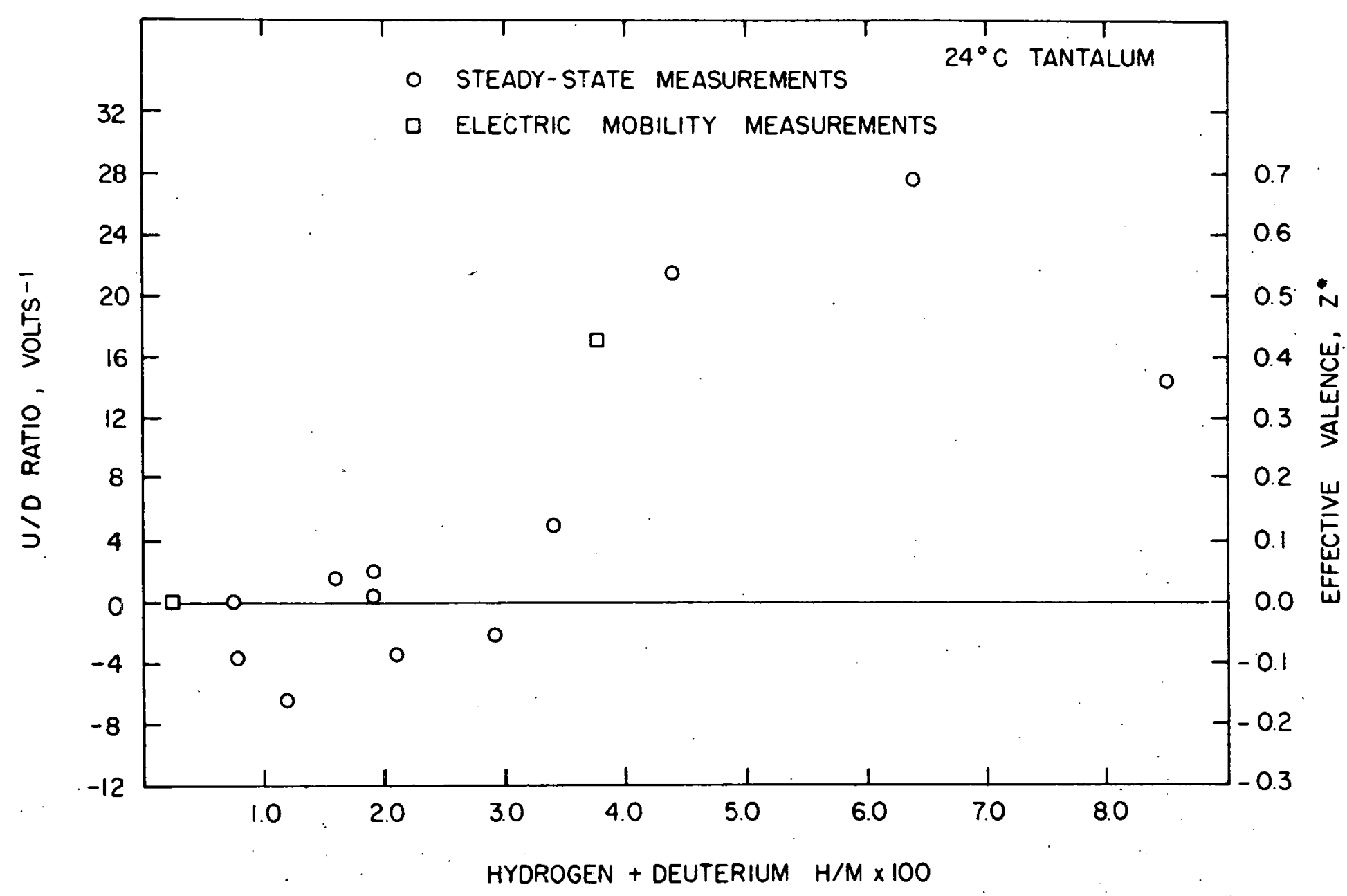

Figure 14. Average U/D ratio of hydrogen and deuterium as a function of total hydrogen isotope concentration at $24^{\circ} \mathrm{C}$ in tantalum 
on the concentration dependence of $U / D$ in tantalum is unknown.

Erckmann and Wipf (2) have measured the effective valence for hydrogen isotopes in niobium and tantalum from $50^{\circ} \mathrm{C}$ to $250^{\circ} \mathrm{C}$. They have included an unspecified thermodynamic correction factor in their calculation of $z^{*}$ which makes direct comparison of experimental data difficult. Because the thermodynamic correction factor varies with concentration and temperature, comparison of the observed temperature and condentration dependences is also difficult. Isotope effects reported by (2) are less than $10 \%$ in niobium, however, in tantalum the effective valence of hydrogen was reported as $30 \%$ larger than that for deuterium.

$$
\text { Niobium-Tantalum Alloy Resistivity }
$$

Figure 15 shows the electrical resistivity of the niobium-tantalum systems as a function of alloy composition at $22^{\circ} \mathrm{C}$. The resistivities of niobium and tantalum compare well with literature values of $14.5 \mathrm{micro} \mathrm{ohm}-\mathrm{cm}$ for niobium and 13.1 micro ohm-cm for tantalum at $22^{\circ} \mathrm{C}(26)$.

The estimated uncertainty in the measurement of the resistivity of the niobium-tantalum alloys is near $2 \%$. Hydrogen 1sotope Resistivities

The specific resistivity of hydrogen and deuterium in vanadium at $22^{\circ} \mathrm{C}$ was measured to be $0.98 \pm 0.02 \mathrm{micro}$ ohm-cm per $(H / M) \times 100$ and $0.90 \pm 0.03$ micro ohm-cm per $(H / M) \times 100$ respec- 


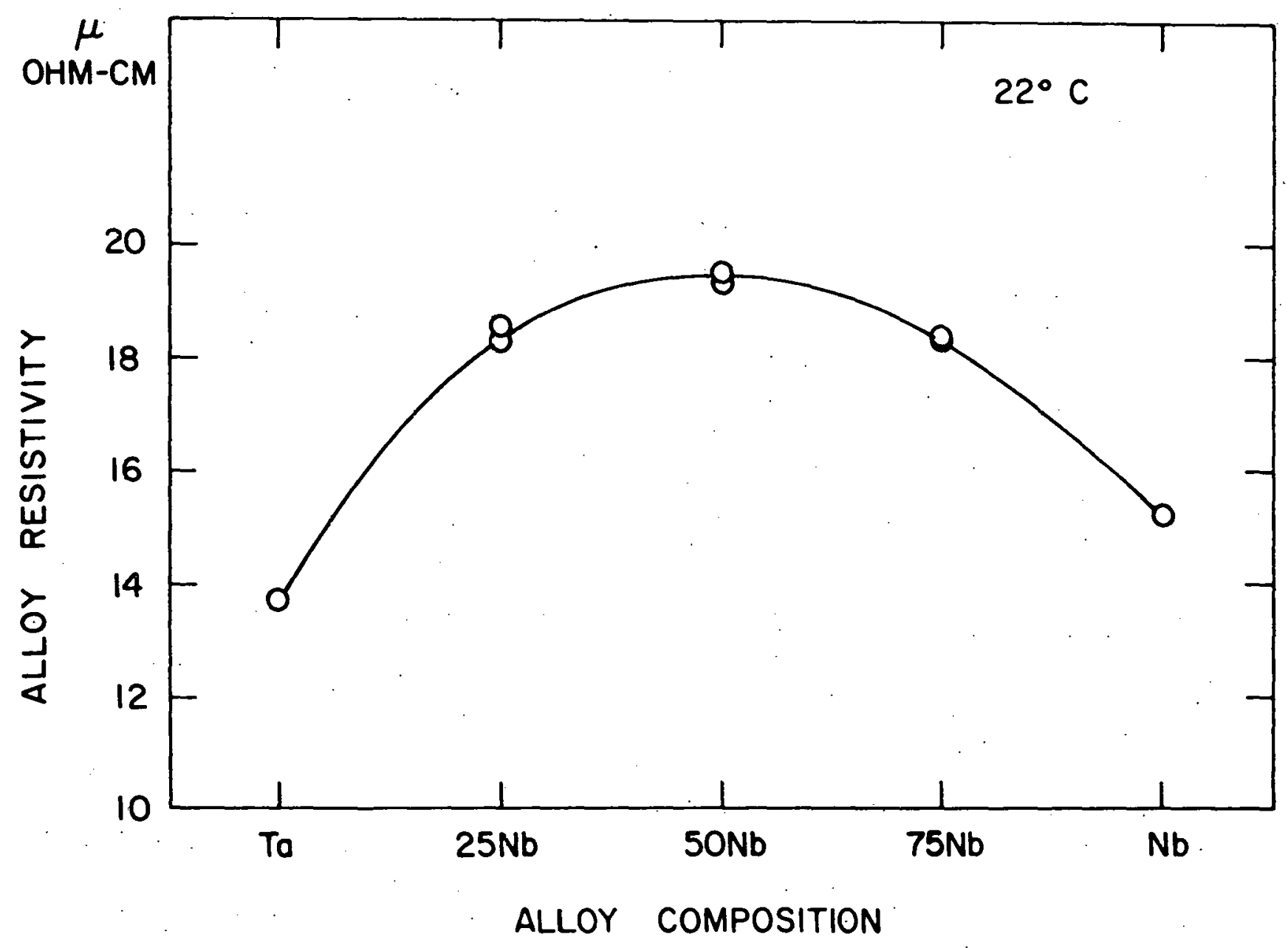

$\stackrel{\infty}{\circ}$

Figure 15. Electrical resistivity of niobium-tantalum alloys at $22^{\circ} \mathrm{C}$ 
tively. These values were obtained by averaging the results of five measurements for each isotope. No concentration dependence in the specific resistivity of hydrogen isotopes in vanadium at $22^{\circ} \mathrm{C}$ was observed. Westlake and Ockers (15) have measured the specific resistivity of hydrogen and deuterium at temperatures up to $170^{\circ} \mathrm{C}$ and for compositions extending to 19 atomic percent. They reported a specific resistivity for hydrogen of 1.1 micro ohm-cm per atomic percent and a value of 0.8 micro ohm-cm per atomic percent for deuterium. The isotope effect reported by (15) is in good agreement with the results of this investigation. The absolute magnitude of the specific resistivities are probably in good agreement if the effect of measuring concentrations in $(\mathrm{H} / \mathrm{M}) \times 100$ or in atomic percent is considered. The specific resistivity on an atomic percent basis will be larger than a specific resistivity based on (H/M)x100 if large concentrations are involved.

Because the solubility limit for hydrogen isotopes in tantalum is near $10(\mathrm{H} / \mathrm{M}) \times 100$ at $22^{\circ} \mathrm{C}$, an opportunity for investigation of the specific resistivity over a wider concentration range exists. Figure 16 shows the increase in resistivity caused by hydrogen and deuterium as a function of solute composition at $22^{\circ} \mathrm{C}$. There appears to be no concentration dependence in the specific resistivity of hydrogen isotopes in tantalum at $22^{\circ} \mathrm{C}$.

The variation in the specific resistivity of hydrogen 


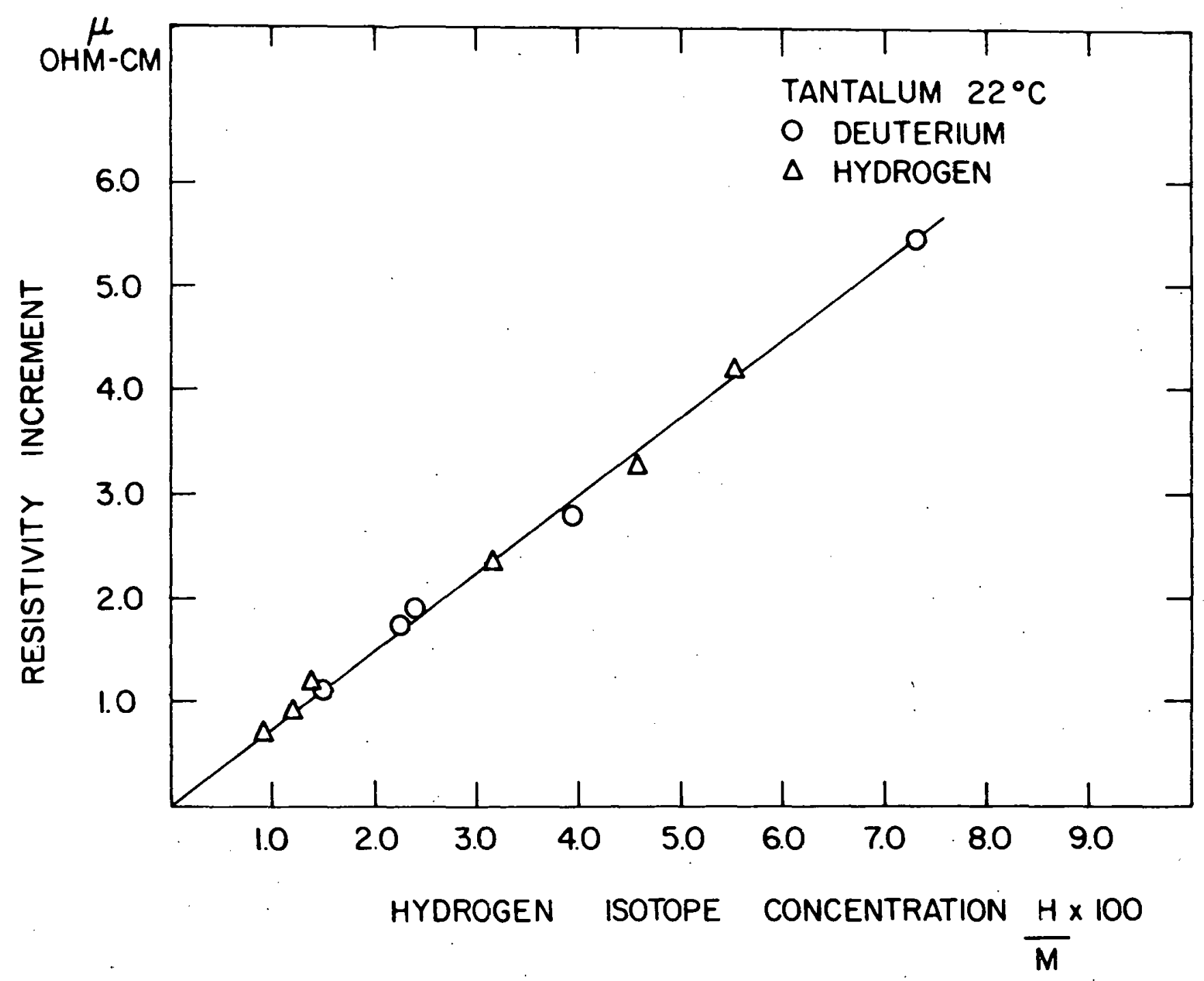

Figure 16. Increase in electrical resistivity of tantalum as a function of hydrogen or deuterium concentration at $22^{\circ} \mathrm{C}$ 
isotopes with niobium-tantalum alloy composition is shown in Figure 17. The specific resistivities given in Figure 17 are the average value of five measurements on each system. The specific resistivity exhibits a uniform decrease as the alloy composition becomes niobium-rich. Isotope effects appear to be greatest at 75-25 alloy compositions and possibly in pure niobium. For all Group VB metals and alloys considered in this investigation, if isotope effects exist, hydrogen always appears to be slightly more effective in scattering charge carriers than is deuterium.

Westlake and Ockers (27) have reported the specific resistivity of hydrogen in niobium as 0.66 micro ohm-cm per atomic percent and $0.63 \mathrm{micro} o \mathrm{hm}-\mathrm{cm}$ per atomic percent for deuterium. The magnitude and isotope effect for the specific resistivity given by (27) are in agreement with those shown in Figure 17. Pryde and Tsong (28) have measured the specific resistivity of hydrogen isotopes in tantalum. Their results were 0.93 micro ohm-cm per $(H / M) \times 100$ for hydrogen and 0.82 micro ohm-cm per (H/M) x100 for deuterium. These results do not agree well with those given in Figure 17 .

$$
\text { Diffusivity of Hydrogen }
$$

Figure 18 shows the experimentally measured diffusion coefficient of hydrogen as a function of hydrogen concentration for the five niobium-tantalum systems at $23^{\circ} \mathrm{C}$. The data for each alloy represent the average of two measurements for 


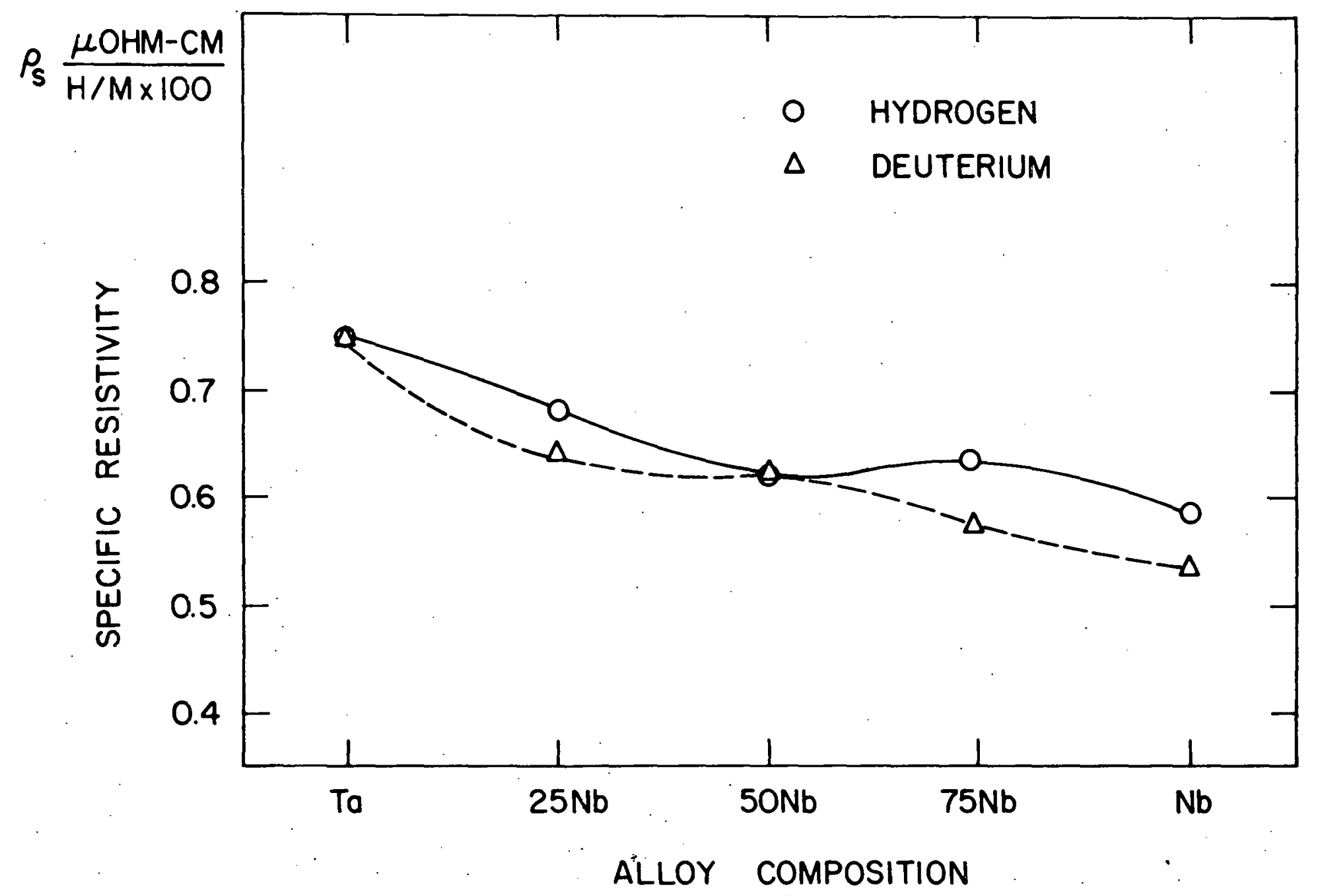

Figure 17. Specific resistivity of hydrogen and deuterium as a function of niobium-tantalum alloy composition at $22^{\circ} \mathrm{C}$ 


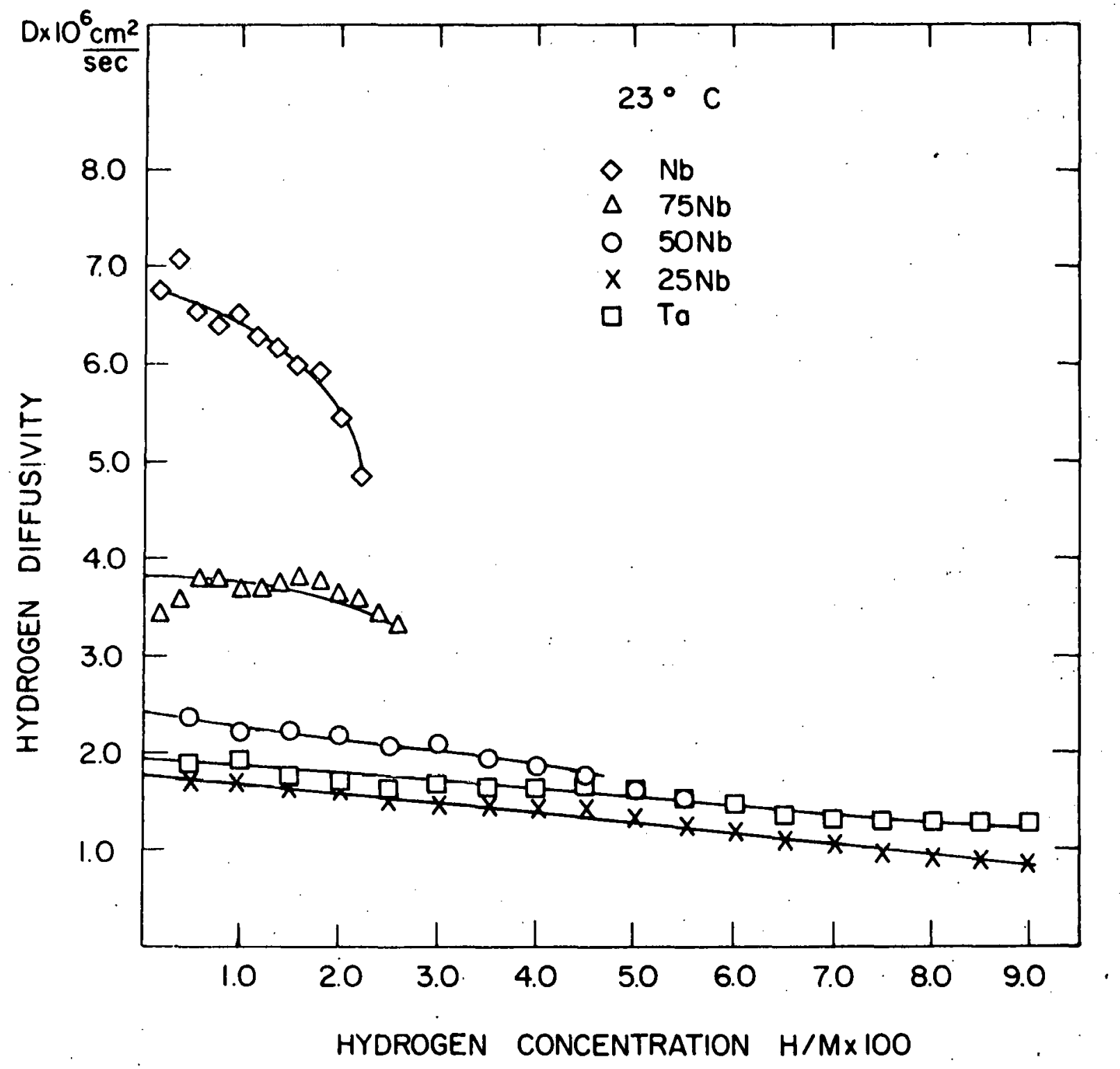

Figure 18. Diffusivity of hydrogen as a function of hydrogen concentration at $23^{\circ} \mathrm{C}$ in niobium-tantalum alloys 
each system. Differing diffusion times were used for each experiment on a given alloy to ensure that the calculated diffusivities were independent of experiment time. For each alloy, the results of the two measurements differed by less than $15 \%$ in all concentration ranges. Figure 19 shows the diffusivity of hydrogen extrapolated to zero solute concentration and also the diffusivity near the midpoint of the solubility range of hydrogen. It appears as if the diffusivity of hydrogen at $23^{\circ} \mathrm{C}$ does not vary linearly with alloy composition. In general, it is difficult to measure diffusivity as a function of solute concentration with good precision. When the Boltzmann-Matano solution is employed to calculate diffusivities, it is necessary to measure the slope of the concentration profile, dc/dx. It is usually quite difficult to obtain concentration profiles of adequate definition such that the slope of the profile can be accurately determined. An example of some experimental data for diffusion of hydrogen in a $75 \mathrm{Ta}-25 \mathrm{Nb}$ alloy is shown in Figure 20. Fortunately, vacuum extraction concentration analysis for hydrogen content is capable of producing concentration profiles of adequate precision such that $\mathrm{dc} / \mathrm{dx}$ is wel1-defined.

A possible source of error in the diffusivities measured by the Boltzmann-Matano technique lies in the location of the $x=0$ interface. An error of this type does not affect $\mathrm{dc} / \mathrm{dx}$, but tends to increase or decrease each $x \cdot d c$ unit of the 


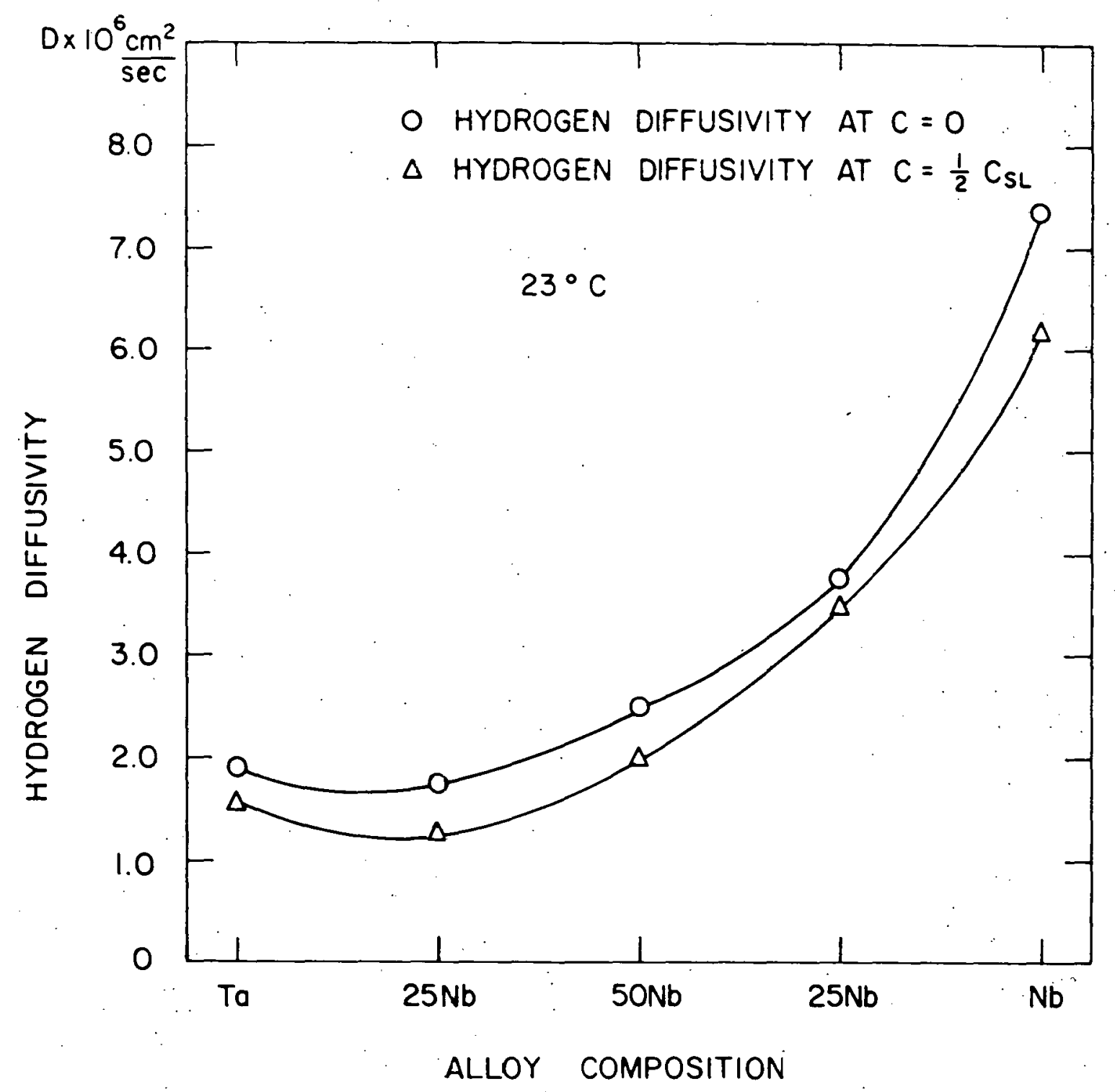

Figure 19. Hydroge: iffusivity at zero hydrogen concentration 


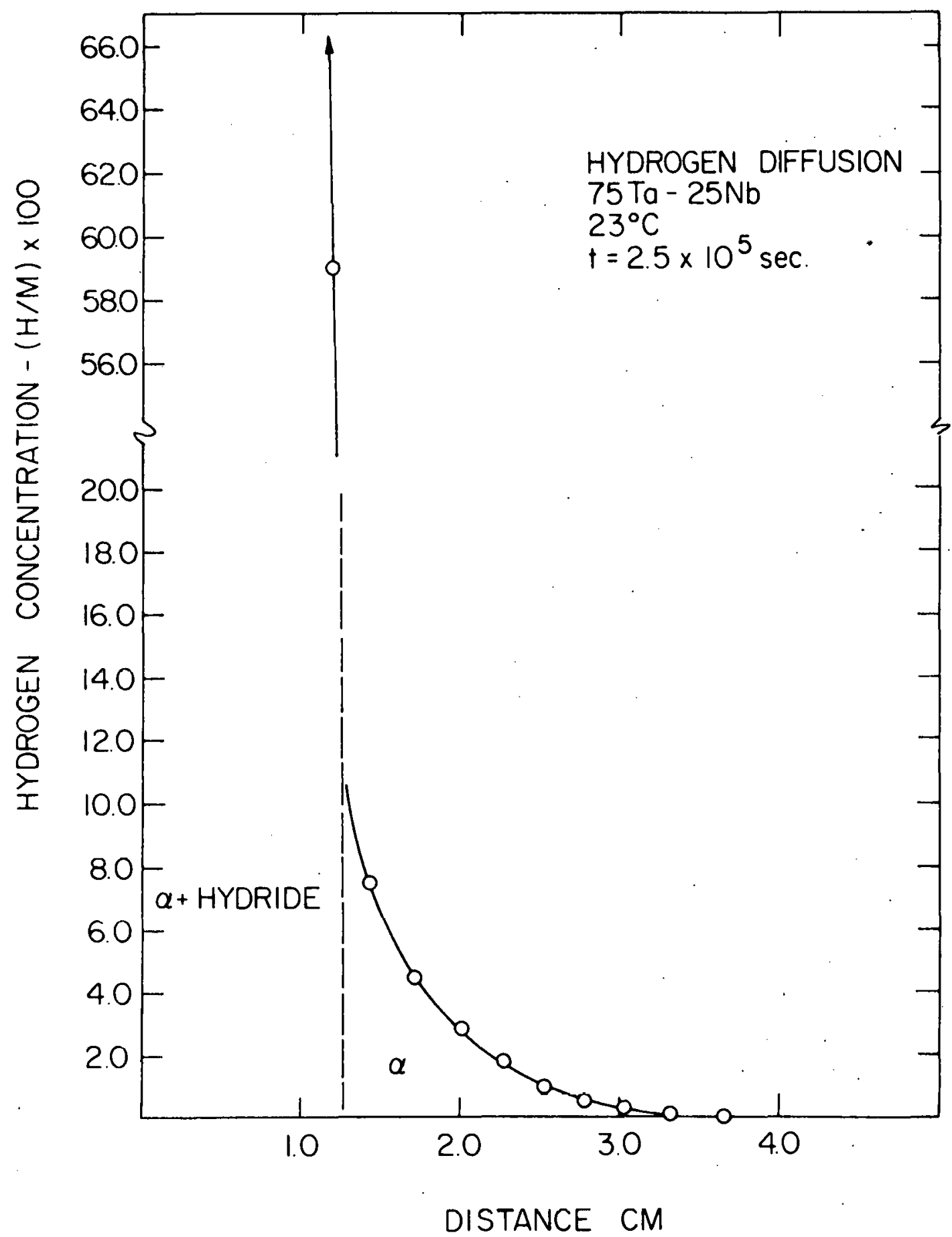

Figure 20. Hydrogen concentration versus distance profile for hydrogen diffusion in $75 \mathrm{Ta}-25 \mathrm{Nb}$ alloy at $23^{\circ} \mathrm{C}$ 
Boltzmann-Matano integration. The net effect of misplacement of the $x=0$ interface is to shift the magnitude of the calculated diffusivity while the concentration dependence of $D$ is not significantly changed. Location of the $x=0$ interface is not difficult for the pure metals where the solubility limit for hydrogen is known (9). The solubility limit for hydrogen in the niobium-tantalum systems was estimated from a linear interpolation of the pure metal solubility limits. Although this assumption is probably not valid, it can be seen from Figure 20 that large errors in the assumed solubility limit do not greatly affect the location of the $x=0$ interface because of the very large concentration gradient near the interface. The shape of the initial two phase-single phase interface may also affect the measured diffusivity of hydrogen. The shape of an interface was determined by electrolytically charging a specimen in the normal manner and then quenching the sample into liquid nitrogen to preserve the initial solute distribution. The specimen was then sectioned and the hydrogen concentration profile determined by hot vacuum extraction analysis. It was found that some hydrogen had diffused into the hydrogen-free section during the 10 minute electrolytic charging process which was done at $60^{\circ} \mathrm{C}$. The concentration profile of hydrogen could be described by a diffusion time of one hour at $23^{\circ} \mathrm{C}$. Because the experimentally used diffusion times are greater than 36 hours, the error introduced by the 
finite time required for electrolytic charging is small. Because the error introduced as a result of the finite time required for electrolytic charging is essentially an error in the measurement of experiment time, only the magnitude and not the concentration dependence of the diffusivity will be. affected.

The zero concentration diffusivity for hydrogen in niobium and tantalum calculated from the data of Schaumann, Volkl and Alefeld (10) is in excellent agreement with those given in Figure 18. The zero concentration diffusivity of hydrogen in niobium from $(10)$ is calculated to be $7.8 \times 10^{-6} \mathrm{~cm}^{2} / \mathrm{sec}$ at $23^{\circ} \mathrm{C}$. The corresponding diffusivity in tantalum is $1.8 \times 10^{-6}$ $\mathrm{cm}^{2} / \mathrm{sec}$.

Kutner and Sosnowska (29) have compiled diffusion data for hydrogen as a function of solute concentration for niobium and tantalum. Their results indicate that hydrogen diffusivity does decrease with increasing solute concentration. The form of the concentration dependence reported by (29) is in agreement with that shown in Figure 18.

As was previously mentioned, Equation 30 predicts that a concentration-independent diffusivity should result from the division of the measured diffusivity by $1+(d \ln \gamma / d \ln c)$. Veleckis and Edwards (30) have calculated values of partial molar entropy and partial molar enthalpy for hydrogen in the Group VB metals. The partial molar entropy, $\Delta \bar{S}$, and the 
partial molar enthalpy, $\Delta \bar{H}$, can be used to calculate a thermodynamic correction factor for hydrogen in niobium and tantalum. The calculated thermodynamic correction factor can then be used to calculate concentration-independent diffusivities from the data for hydrogen diffusivity appearing in Figure 18. The data of Veleckis and Edwards (30) were obtained near $500^{\circ} \mathrm{C}$, however, the calculated quantities, $\Delta \overline{\mathrm{H}}$ and $\Delta \overline{\mathrm{S}}$, should be relatively independent of temperature. Assuming $\Delta \overline{\mathrm{H}}$ and $\Delta \overline{\mathrm{S}}$ to be temperature-independent, the data of (30) suggest that $\Delta \bar{H}$ and $\Delta \bar{S}$ can be analytically expressed as functions of concentration as

$$
\Delta \overline{\mathrm{H}}=\mathrm{A}_{\mathrm{H}}+\mathrm{B}_{\mathrm{H}} \cdot \mathrm{C}
$$

and

$$
\Delta \vec{S}=A_{S}+B_{S} \cdot \ln c .
$$

$A_{H}, B_{H}, A_{S}$ and $B_{S}$ are constants which were determined from a least-squares analysis of the data of (30) for concentrations be low $10(\mathrm{H} / \mathrm{M}) \times 100$.

Using the relation between the partial molar quantities and the activity coefficient

$$
\Delta \overline{\mathrm{H}}-\mathrm{T} \Delta \overline{\mathrm{S}}=\mathrm{RT} \text { In } \gamma \cdot \mathrm{C}
$$

an expression for the thermodynamic correction factor can be derived.

$$
1+\frac{d \ln \gamma}{d \ln c}=\frac{c \cdot B_{H}}{R T}-\frac{B_{S}}{R} .
$$

Equation 34 has been used to compute the thermodynamic correc- 
tion factor as a function of hydrogen concentration for niobium and tantalum at $23^{\circ} \mathrm{C}$. Figure 21 shows the measured hydrogen diffusivity and the calculated diffusivity which should be concentration-independent. The use of the thermodynamic correction factor does not remove the concentration dependence from the measured diffusivity of hydrogen. It appears as if this method of determining the thermodynamic correction factor does not produce reasonable correction factors near $23^{\circ} \mathrm{C}$. It was for this reason that a thermodynamic correction factor was not used in the calculation of the effective valence from steady-state electromigration data.

Internal Consistency of the Experimental Data for $U, D$ and $U / D$

The ratio of the electric mobility to the solute diffusivity was measured directly with the steady-state electromigration technique. The diffusivity of hydrogen in the Group VB metals is available in the literature (10) and has also been measured at $23^{\circ} \mathrm{C}$ as a function of hydrogen concentration in this research. The electric mobility was directly measured with the resistance technique. Thus, the internal consistency of the three transport parameters can be evaluated by comparison of the directly measured $U / D$ ratio with the ratio computed from electric mobility and diffusivity data.

For hydrogen and deuterium in vanadium and niobium, there is reasonable agreement between the calculated and 


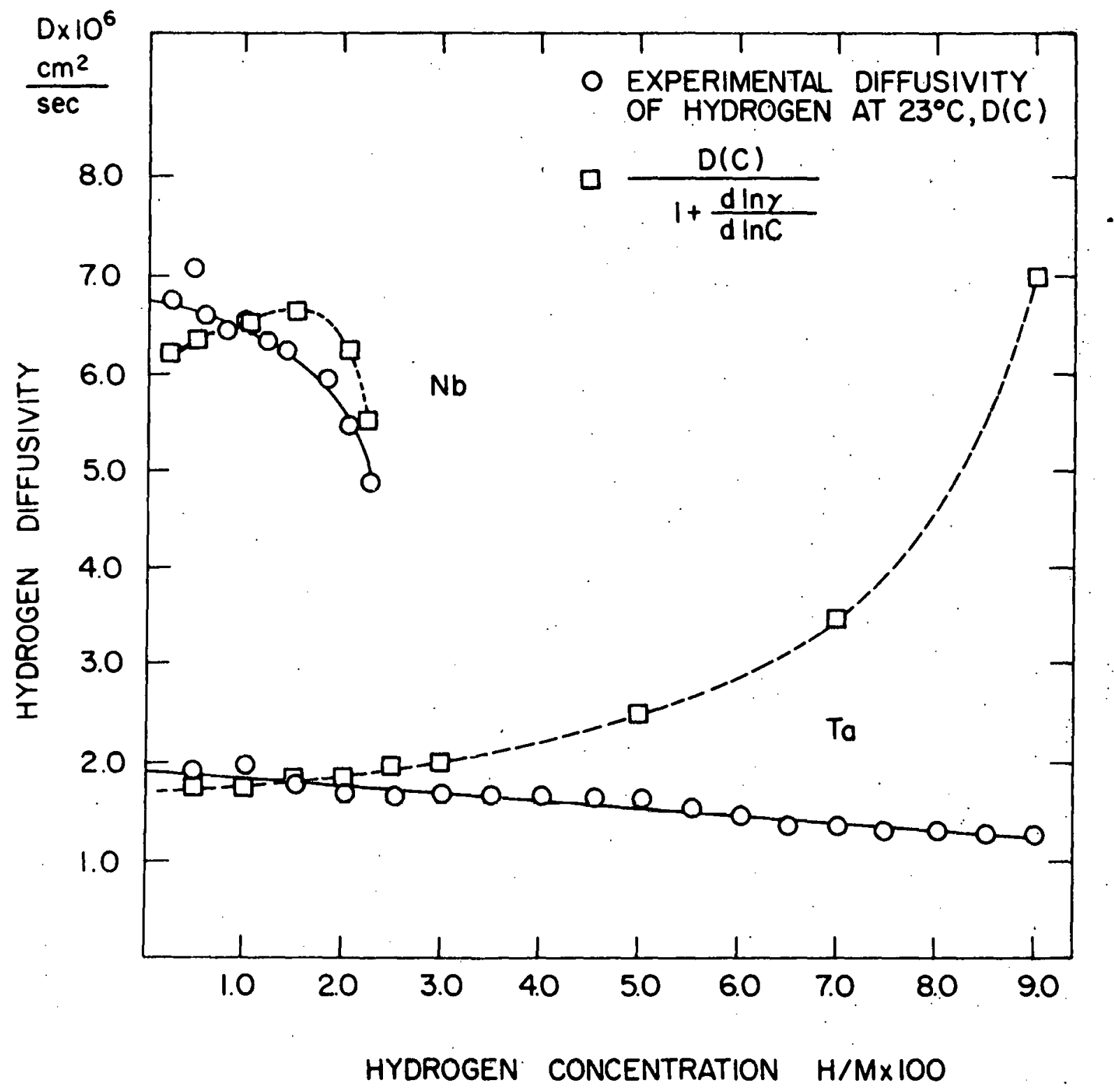

Figure 21. Experimentally measured hydrogen diffusivity, $D(c)$, and $D(c) /[1+d$ in $/ d$ in $c]$ as a function of hydrogen concentration at $23^{\circ} \mathrm{C}$ for niobium and tantalum 
experimental values of $U / D$. The largest differences between the directly.measured $U / D$ ratio and the ratio of the measured electric mobility to hydrogen diffusivity calculated from (10) are near $35 \%$. This is not unreasonable agreement when the individual uncertainty in each of the three measurements is considered. The electric mobility and diffusivity measurements are subject to $15 \%$ error. The steady-state determination of $U / D$ can be done with an estimated uncertainty of near $10 \%$. Thus, the calculated and experimental U/D ratios may be expected to differ by as much as $40 \%$.

For niobium and vanadium, there is agreement between the three transport measurements with regard to the 1sotope effect in $U / D$. In each instance, deuterium has a U/D ratio which is larger than that for hydrogen. The isotope effect measured by the steady-state technique is, however, much more reliable than the isotope effect from the difference of $U / D$ ratios calculated from individual electric mobility and diffusivity measurements.

In niobium, $U$ and $U / D$ were found to be independent of solute concentration. The diffusivity of hydrogen appeared to decrease by $30 \%$ over the range of hydrogen solubility at $23^{\circ} \mathrm{C}$. These three concentration dependences are not consistent with each other.

The determination of the concentration dependence of $U / D$ by the steady-state method appears to be the most reliable 
measurement. The concentration dependence in the diffusivity of hydrogen is the most difficult concentration dependence to measure. However, the decrease in hydrogen diffusivity with increasing hydrogen concentration is supported by other measurements (29). The direct measurement of the electric mobility is subject to a relatively large uncertainty and also, $U$ was not measured over a wide range of concentrations. Considering these observations, the most probable situation is that $U / D$ is independent of solute concentration to within $10 \%$, except for tantalum. The diffusivity of hydrogen most likely decreases with increasing hydrogen concentration, although the magnitude of this decrease may not be as large as indicated in Figure 18. The electric mobility must then also decrease with increasing solute concentration in such a manner that $U / D$ be nearly independent of solute concentration. Because of the uncertainty associated with each measurement, it is believed that this set of proposed concentration dependences for the three transport parameters is not in serious disagreement with the experimental data. 


\section{DISCUSSION}

Phenomena involving interstitial defects in metals can generally be discussed in terms of vibrational and electronic interactions between the solute and the host lattice. When considering isotope effects; it is quite natural to ascribe the mass dependence of a process to the differing vibrational characteristics of the chemically equivalent solutes. The relative magnitude of an interstitial effect is generally a more complex property of the system arising from both electronic and vibrational interactions.

Consider the increase in electrical resistivity due to a unit concentration of an interstitial in a metal. For estimation of the magnitude of the specific resistivity, Linde's rule, relating the valence difference of solute and solvent and the impurity resistivity, could be used to estimate the specific resistivity of different interstitials in a given host. Isotope effects in the specific resistivity could be considered in terms of the difference between the ground state vibrational mode for the isotopes. The ground state vibrational amplitude for the light isotope would be expected to be larger than the corresponding amplitude for the heavier isotope. Thus, it may be anticipated that the lighter isotope will be more effective in scattering conduction electrons than the heavier isotope.

The results of the specific resistivity measurements 
indicate that these general arguments have some validity for hydrogen isotopes dissolved in Group VB metals. The magnitude of the specific resistivity for hydrogen isotopes is similar for each of the Group VB metals, ranging from 0.6 to $1.1 \mu \Omega$ $\mathrm{cm} /(\mathrm{H} / \mathrm{M}) \times 100$. This is considerably smaller than the specific resistivity associated with other interstitials. Oxygen has been found to contribute $5.2 \mu \Omega-\mathrm{cm} /$ atomic percent to the resistivity of vanadium (31) while nitrogen adds $10 \mu \Omega-\mathrm{cm} /$ atomic percent to the resistivity of niobium (32). Thus, electronic effects appear to be very important in determining the magnitude of the specific resistivity.

The direction of the isotope effect in the specific resistivity is in agreement with the vibrational amplitude argument concerning the relative scattering cross-sections of hydrogen isotopes for conduction electrons (33). Where isotope effects are found $(\mathrm{V}, \mathrm{Nb}, 25 \mathrm{Nb}-75 \mathrm{Ta}, 75 \mathrm{Ta}-25 \mathrm{Nb})$, the specific resistivity associated with hydrogen is larger than that for deuterium.

The experimental data suggest that the magnitude of the isotope effect in the specific resistivity tends to decrease as the mass of the host metal atom increases. The increase in lattice atom mass could be expected to cause hydrogen isotopes to become increasingly decoupled from the lattice vibrational modes (34). The formation of local modes can, alternatively, be viewed as a deepening of the potential well in 
which the interstitial is confined. The increase in activation energy for hydrogen diffusion from $0.050 \mathrm{eV}$ in vanadium to $0.140 \mathrm{eV}$ in tantalum (10) may be taken as evidence for the increase in the depth of the potential well as the mass of the lattice atoms is increased.

Assuming that a harmonic oscillator potential, $\mathrm{k} \cdot \mathrm{x}^{2} / 2$, can adequately describe the potential well in the region where hydrogen and deuterium reside at ambient temperatures, the classical turning point, or vibrational amplitude, of hydrogen isotopes in the ground state can be written as

$$
x=\left(h^{2} / k \cdot m\right)^{1 / 4}
$$

where $x$ is the classical turning point, $m$ is the mass of the isotope, $h$ is Planck's constant and $k$ is the constant of proportionality for the parabolic well. Because the lattice. parameters of vanadium, niobium and tantalum differ by less than $10 \%$, the deepening of the potential well as the mass of the lattice atom increases requires that $k$ also increase with lattice atom mass.

Differentiating Equation 35 with respect to $\mathrm{m}$ yields

$$
\Delta x=-h^{1 / 2} \cdot \Delta m /\left(4 \cdot k^{1 / 4} \cdot m^{3 / 4}\right) \text {. }
$$

For a given change in isotopic mass, $\Delta \mathrm{m}$, the change in the classical turning point caused by $\Delta \mathrm{m}$ will be larger at low values of $k$ than for larger values of $k$. Thus, the difference in the classical turning point for hydrogen and deuterium should be greater in vanadium than in tantalum. Assuming that 
the classical turning point of a hydrogen isotope is related to the specific resistivity of that isotope, isotope effects in the specific resistivity should be the largest in vanadium and decrease as the mass of the lattice atom increases. This prediction is in agreement with the specific resistivity data for hydrogen isotopes in vanadium, niobium and tantalum.

The electrical resistivity of the niobium-tantalum alloys appears to be a rather classic example of the resistivity for a binary alloy system. The analysis of Nordheim (35), can be used to predict the resistivity of a binary alloy as

$$
\Delta \rho=A \cdot f_{\mathrm{Nb}} \cdot\left(1-f_{\mathrm{Nb}}\right) \cdot
$$

$A$ is a constant, $f_{\mathrm{Nb}}$ is the atomic fraction of niobium in the alloy and $\Delta \rho$ is the increase in electrical resistivity above that predicted by a linear interpolation of the pure metal resistivities. The constant, $A$, can be interpreted as the increase in resistivity due to the first small addition of solute to the pure metal. Equation 37 has been simplified somewhat from its original form due to the nearly constant unit cell volume across the entire niobium-tantalum alloy range. Figure 22 represents the experimentally determined value of $\Delta \rho$ which has been calculated from Figure 17. The solid 1ine in Figure 22 indicates the best fit of Equation 37 to the experimental data. The maximum resistivity increase per unit alloy addition, $A$, was found to be $0.21 \mu \Omega-\mathrm{cm} /$ atomic percent. 


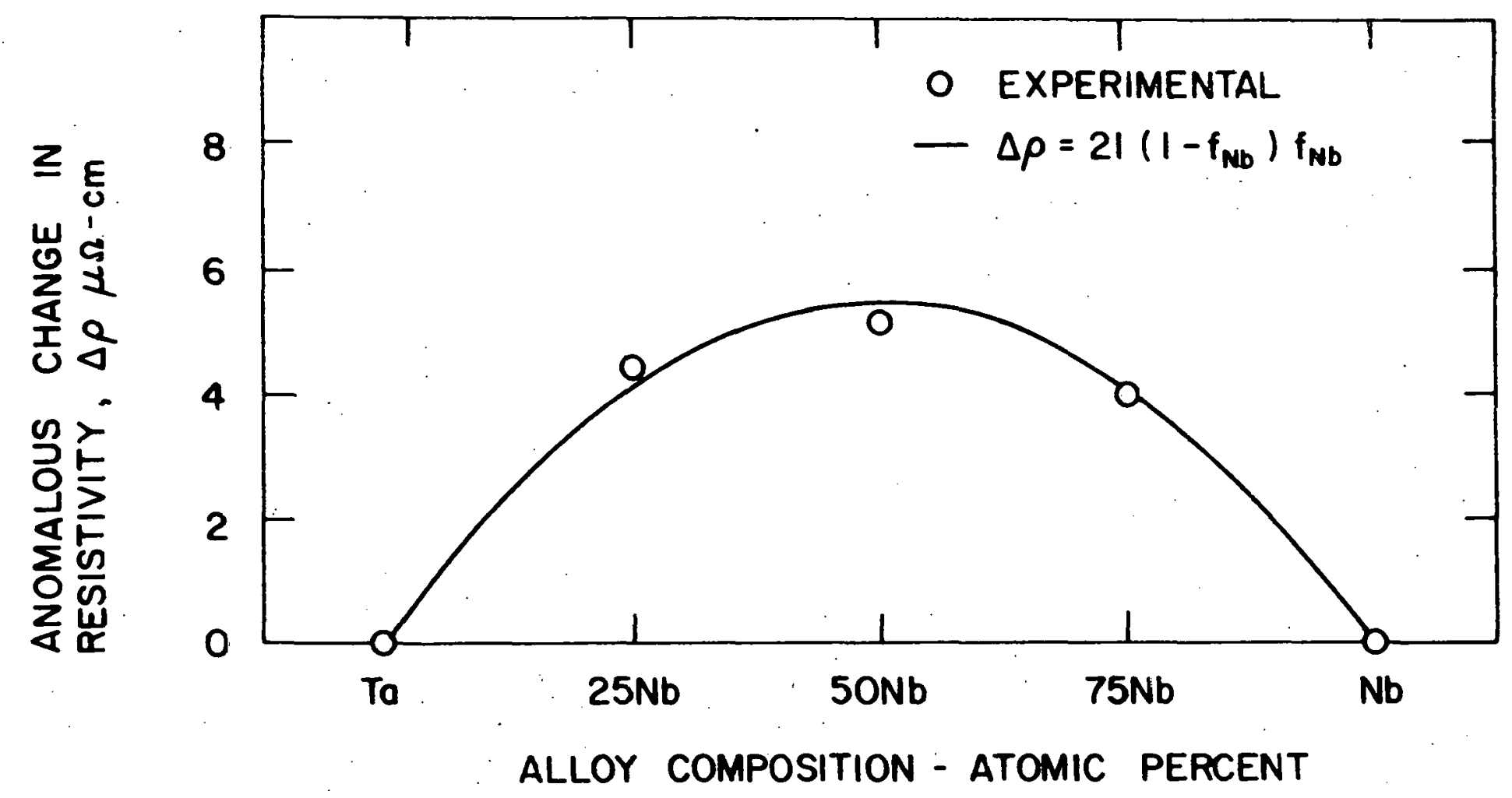

•

Figure 22. Anomalous change in electrical resistivity of niobium-tantalum alloys at $22^{\circ} \mathrm{C}$ 
The diffusivity of hydrogen in the niobium-tantalum systems also differs from a linear dependence on alloy composition. Figure 23 shows the measured hydrogen diffusivity minus the diffusivity obtained from a linear interpolation of the pure metal diffusivities. The anomalous change in hydrogen diffusivity is reminiscent of the behavior observed in the electrical resistivity of the niobium-tantalum alloys. Both the anomalous change in hydrogen diffusivity and electrical resistivity reach a maximum magnitude near $45 \%$ of the linearly interpolated value.

Because the behavior of the diffusivity and electrical resistivity is similar, it might be postulated that alloy composition affects these processes in the same manner. The electrical resistivity deviation was shown to obey the Nordheim relation for the scattering of conduction electrons in a binary alloy. A similar relation can be derived for the scattering of phonons in an alloy consisting of chemically equivalent atoms of differing mass (36). The consideration of niobium-tantalum alloys as an isotopic mixture leads to a $f_{N b}\left(1-f_{N b}\right)$ dependence for phonon scattering.

The connection between phonon scattering and hydrogen diffusivity is not as simple as the relation between electron scattering and electrical resistivity. Although the exact means by which a change in phonon scattering could cause a variation in solute diffusivity cannot be ascertained from 


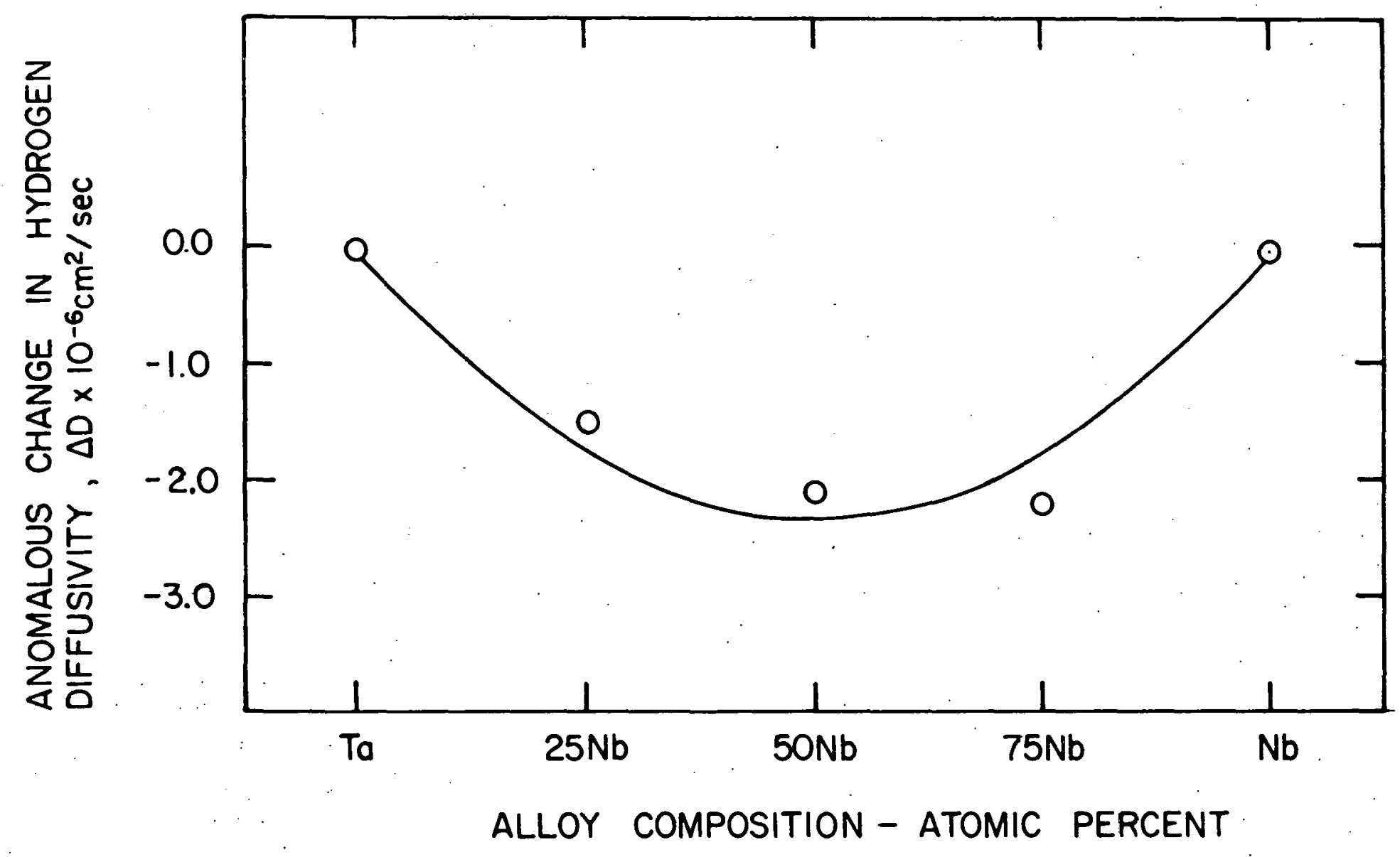

蒕

Figure 23. Anomalous change in hydrogen diffusivity in niobium-tantalum alloys
at $23^{\circ} \mathrm{C}$ 
these experiments, there are several ways in which the vibrational character of the system could influence diffusion. Thermal vibrations are the source of the necessary activation energy which the solute atom requires in order to cross the saddle position. A change in phonon mean free path would probably influence the rate of energy transfer to solute atom and, thus, vary the successful diffusive jump frequency. Also, the vibrational aspects of the host lattice will have a bearing on the geometry of the jump path. Vibrational amplitudes and the frequency of vibration for the host metal will influence the process of interstitial diffusion by controlling the number of opportunities which an interstitial atom will have to pass between the atoms which obstruct the jump path. Thus, vibrational characteristics may influence the activation energy for diffusion through control of the lattice atoms which must be moved from the jump path during the diffusive jump. Because it has not been determined how the activation energy and pre-exponential term for hydrogen diffusivity vary as a function of alloy composition, it is difficult to speculate on the exact role of phonon scattering in the process of hydrogen diffusion.

The non-linear dependence of phonon properties is not supported by the observed variation in the superconducting transition temperature with alloy composition. The temperature at which a material begins to exhibit superconducting 
behavior is strongly dependent on electron-phonon interactions: Thus, a major change in phonon scattering behavior as a function of alloy composition should be reflected in an anomalous behavior of the superconducting transition temperature. However, the superconducting transition temperature has been shown to be a linear function of niobium-tantalum alloy composition (37). This result appears to indicate that the variation in phonon properties with alloy composition is somewhat unclear.

The processes of electrical resistivity, diffusivity and solute resistivity have been discussed in terms of gross changes in electronic and vibrational interactions between solute and solvent which are caused by variation of the host lattice through alloying. Although the minute details of the scattering and activation events are not known, statistical averaging appears to compensate for the range of possible interactions. Thus, an average value of such microscopic parameters as electron or phonon mean free path, activation energy or vibrational frequency and amplitude are generally quite useful for discussion of electrical resistivity and diffusivity changes which occur when the host metal is varied.

When considering the electromigration process, it might be expected that vibrational and electronic arguments could be used to explain the observed behavior of hydrogen isotopes in response to an applied electric field. Vibrational and elec- 
tronic arguments were quite useful for describing magnitudes and isotope effects for transport processes such as electrical conduction and diffusion. If this type of reasoning is valid for electromigration, Equation 9, which describes the electromigration force in terms of resistivity and electrostatic. components, should prove useful for analysis of observed electromigration behavior.

With reference to niobium-tantalum alloys, it would be expected that any electrostatic component of the electromigration force would not be a strong function of alloy composition. The constant, $z_{m}$, in Equation 9 can be interpreted as the number of valence electrons per matrix atom. A less formal approach would let $z_{m}$ be characteristic of some electronic property of the solute-solvent system which determines the magnitude of the electron wind force. Either interpretation of $z_{m}$ would predict nearly identical values of this constant for niobium and tantalum because of the extreme similarity of the two metals. According to Equation 9, the major variable which should determine the magnitude of the effective valence for hydrogen isotopes in niobium-tantalum alloys is $\rho_{\mathrm{s}} / \rho_{\mathrm{m}}$.

The ratio of the solute resistivity to the resistivity of the metal can be calculated from the data given in Figures 15 and 17. $\rho_{s} / \rho_{m}$ is shown as a function of alloy composition in Figure 24. It is apparent that the electron wind force should 


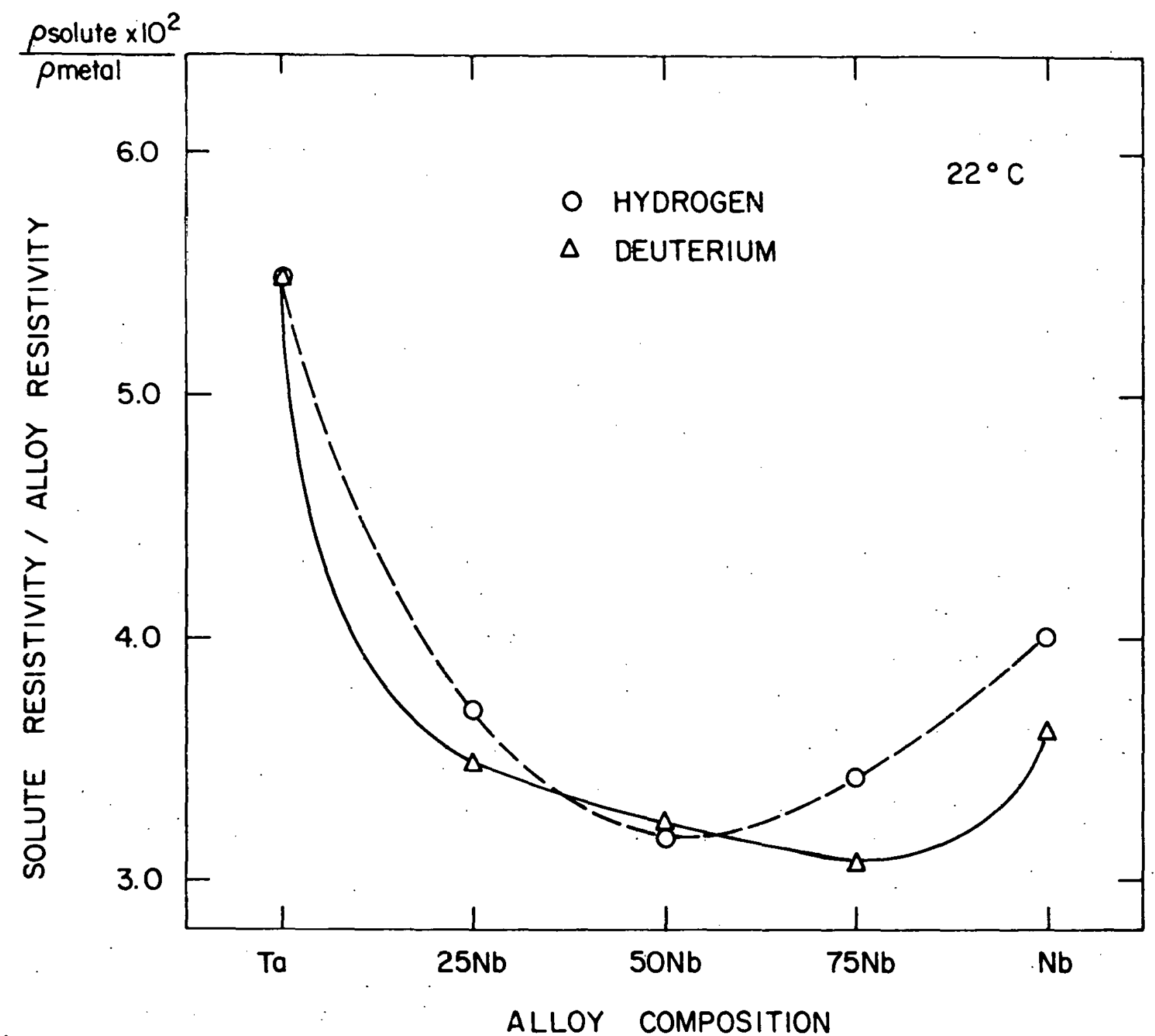

๖응

Figure 24. Ratio of the specific rey COMPOSITION

resistivity of the 
pass through a minimum as the niobium-tantalum alloy range is traversed.

As was observed in Figures 10, 11 and 12, a minimum effective valence was not detected, but rather $z^{*}$ was found to be nearly a linear function of alloy composition. The surprising aspect of the electromigration of hydrogen in niobium and tantalum is that the effective valences do differ greatly. This was not anticipated by Equation 9 and cannot be explained by any known difference between the electronic or vibrational character of niobium and tantalum. It must be concluded that the use of Equation 9 for description of the electromigration process of hydrogen in niobium-tantalum alloys seems limited because this expression does not specify any variable which could account for the large difference between the effective valence in niobium and tantalum. The validity of the quantity $\rho_{s} / \rho_{m}$ for describing changes in the electron wind force cannot be ascertained from these experiments. The variation in $Z^{*}$ by a factor of four upon going from tantalum to niobium completely overshadows any change in $z^{*}$ caused by the $45 \%$ decrease in $\rho_{s} / \rho_{m}$. No conclusion can be drawn regarding the presence of a direct electrostatic force on hydrogen isotopes in niobium, vanadium or tantalum.

The inability to rationalize the behavior of the electromigration force in terms of other observable changes in the system may be interpreted as evidence for a major difference 
which exists between electromigration and other transport processes such as diffusion or electrical conduction. Electromigration is a much more subtle phenomenon than other transport processes.

The number of diffusive jumps which are biased in a given time interval can be calculated from the velocity of migration and the microscopic jump distance. Using an electric mobility of $1 \times 10^{-4} \mathrm{~cm}^{2} /$ volt-sec, an electric field of $5 \times 10^{-3} \mathrm{volts} / \mathrm{cm}$ and a jump distance of $2 \times 10^{-8} \mathrm{~cm}$, it can be calculated that 25 jumps of a hydrogen atom are biased per second as a result of the applied potential. This frequency is extremely low in comparison with important frequencies for other transport processes. Characteristic rates for conduction electron scattering are near $10^{15}$ per second while phonons are scattered with typical frequencies of $10^{12}$ per second. For diffusion of hydrogen, typical successful jump frequencies are near $10^{10}$ per second. Therefore, it can be estimated that 3 diffusive jumps in $10^{9}$ are biased by electromigration.

It is quite interesting to note that although the biasing of diffusive jumps occurs quite infrequently on an atomistic time scale, the observed transport behavior appears to be describable in terms of classical mobility expressions. The validity of classical mobility relations generally requires that a continuous force act on the migrating particle. In the case of electromigration, this assumption is made when 
the force on the particle is related to the magnitude of the electric field by the effective valence. If the electromigration force is electrostatic, the assumption of a continuous force is valid. It is not immediately obvious that a momentum transfer force, such as the electron wind, will be continuous.

To estimate the number of ballistic interactions which occur per unit time, a calculation similar to that by Huntington and Grone (8) can be carried out. The density of electrons in a metal is near $10^{23}$ per $\mathrm{cm}^{3}$. Because only electrons near the Fermi surface can transfer momentum to the solute, there are perhaps only $10^{21}$ electrons per $\mathrm{cm}^{3}$ which could bias a diffusive jump. The average speed of these electrons will be near the Fermi velocity, or around $10^{8} \mathrm{~cm} / \mathrm{sec}$. Assuming a scattering cross-section of a hydrogen atom for conduction electrons of $10^{-15} \mathrm{~cm}^{2}$, the number of possible momentum exchanges would be near $10^{14}$ per second. Combining this frequency with the successful jump frequency of $10^{10}$ per second yields an estimate of $10^{4}$ electron-hydrogen momentum exchanges while the solute is in a single equilibrium site.

It should be remembered that the ballistic interaction cannot bias a diffusive jump unless the solute atom is near the top of the potential well. The energy supplied to the solute atom by the interaction with the electron must make the total solute energy greater than the activation energy for diffusion if a biased jump is going to occur. The kinetic 
energy which an electron can transfer to the hydrogen atom is only a minute fraction of the activation energy necessary for diffusion. This can be seen from the energy gained by an electron when it is accelerated by the electric field over a distance of one mean free path. Using an electric field of $5 \times 10^{-3}$ volts $/ \mathrm{cm}$ and a mean free path of $10^{-7} \mathrm{~cm}$ the electron will gain approximately $5 \times 10^{-10} \mathrm{eV}$. For all practical purposes, the hydrogen atom must be struck by the conduction electron while it is at the activated site if a biased jump is to occur. Assuming an activation energy of $0.10 \mathrm{ev}$ for hydrogen diffusion, the fraction of hydrogen atoms which are at the activated site at $30^{\circ} \mathrm{C}$ can be estimated from $\exp (-0.10 / 0.025)$ $=10^{-2}$. Of the $10^{4}$ electron-hydrogen momentum exchanges occurring in a single equilibrium site, $10^{2}$ will occur with a hydrogen atom possessing sufficient energy that a biased diffusive jump is possible. Considering that only 3 diffusive jumps in $10^{9}$ are biased by electromigration, it can now be estimated that about one electron-hydrogen interaction in $10^{11}$ interactions which are capable of biasing a diffusive jump will actually do so. It appears that a significant number of electrons do strike the hydrogen atom when it is near the activated site and thus, the electron wind force should be relatively continuous. However, the electron-hydrogen interactions appear to be terribly inefficient in biasing the diffusive jump process. 
From a theoretical standpoint, the inefficiency in the biasing of diffusive jumping would seem to make a calculation of the effective force on a solute nearly impossible. The ability to calculate whether one or ten jumps in $10^{11}$ will be biased would require a very detailed knowledge of electron and solute states. The simple averaging procedures which are useful in explaining the transport processes of diffusion and electrical conduction may be expected to be of little help in describing the very subtle process of electromigration. 


\section{CONCLUSIONS}

Electrical resistivity and hydrogen diffusivity were found to be non-linear functions of niobium-tantalum alloy composition. The non-linear dependence of these properties can best be explained by simple Nordheim arguments which consider the scattering of electrons and phonons in a binary alloy. The effective valence and specific resistivity of hydrogen and deuterium were found to be linear functions of niobium-tantalum alloy composition. The linear variation of these properties is probably the result of the averaging of two slightly different electronic structures when niobium and tantalum are alloyed. Isotope effects in the specific resistivity of hydrogen and deuterium may be rationalized on the basis of the dependence of vibrational amplitude for a particle in a potential well on the mass of the particle and the shape of the potential well.

The ratio of the electric mobility of hydrogen to that of deuterium in niobium and vanadium is greater than the classically expected value of 1.41 at $30^{\circ} \mathrm{C}$. The effective valence of deuterium is, however, greater than that for hydrogen as calculated from electric mobility data and also as directly measured with steady-state techniques.

The dependence of the electron wind force on the ratio of solute-to-host electrical resistivities is not clear. While the electron wind force for deuterium is measured to be 


\section{3}

larger than that for hydrogen, the increase in resistivity caused by hydrogen is greater than for deuterium in niobium and vanadium. No conclusion regarding the dependence of the electron wind force on $\rho_{s} / \rho_{m}$ can be drawn from the variation of the effective valence of hydrogen and $\rho_{s} / \rho_{m}$ as a function of niobium-tantalum alloy composition. Any variation in $z^{*}$ due to a change in $\rho_{s} / \rho_{m}$ as a function of alloy composition is overshadowed by a much larger change in $Z^{*}$ caused by an intrinsic difference in the electromigration force on hydrogen in niobium and tantalum.

Electromigration appears to be a very subtle process in comparison with other transport properties such as diffusion or electrical conduction. The extreme subtlety of the electromigration process may account for the apparent insensitivity of the effective force to the gross vibrational or electronic character of the solute-solvent system. 


\section{ACKNOWLEDGEMENTS}

I would like to express my appreciation and indebtedness to Dr. David T. Peterson for his patient guidance and support during the course of this research.

Thanks is given to Mr. Gerald Flesch for the mass spectrographic analysis of hydrogen isotope gas. Thanks are also extended to Messirs. A. Johnson, H. Baker and L. Reed for their technical assistance and to Dr. J. Patterson, Dr. S. Liu and Dr. J. Verhoeven for many valuable discussions.

A special thanks belongs to my parents for their untiring encouragement of my desire to learn. 


\section{B IBL IOGRAPHY}

1. R. E. Einziger and H. B. Huntington, J. Phys. Chem. Solids, $\underline{35}, 1563$ (1974).

2. V. Erckmann and H. Wipf, Phys. Rev. Letters, 37, 341 (1976).

3. F. Skaupy, Verh. Dtsch, Phys. Ges., 16, i56 (1914).

4. H. Wever and W. Seith, Z. Elektrochem., 59, 942 (1955).

5. C. Bosvieux and J. Friedel, J. Phys. Chem. Solids, 23, 123 (1962).

6. H. B. Huntington, Trans. of the Metallurgical Society of AIME, 245,2571 (1969).

7. V. B. Fiks, Soviet Phys. Solid State (Eng. Transl.), 1 , 14 (1959).

8. H. B. Huntington and A. R. Grone, J. Phys. Chem. Solids, 20, 76 (1961).

9. C. V. Owen and T. E. Scott, Metallurgical Trans., $\underline{3}$, 1715 (1972).

10. G. Schaumann, J. Volk1 and G. Alefeld, Phys. Status Solidi, $\underline{42}, 401$ (1970).

11. C. Wagner and G. Heller, Z. Physik. Chem. Abt. B, 46, $242(1940)$.

12. J. Knaak and W. Eichenauer, Z. Naturforsch., 23A, 1783 (1968).

13. J-F. Mareche, J-C. Rat and A. Herold, J. Chimie Physique, 73, 1 (1976).

14. R. A. Oriani and 0 . D. Gonzalez, Trans. of the Metallurgical Society of AIME, 239, 1041 (1967).

15. D. G. Westlake and S. T. Ockers, Metallurgical Trans., 4 , 1355 (1973).

16. G. G. Libowitz, "The Solid-State Chemistry of Binary Metal Hydrides," W. A. Benjamin, Inc., New York, N.Y., 1965, p. 27.

17. G. Alefeld, Phys. Status Solidi, $\underline{32}, 67$ (1969). 
18. C. P. Flynn and A. M. Stoneham, Phys.Rev. B. 1, 3966 $(1970)$.

19. D. T. Peterson and V. G. Fattore, Analytical Chemistry, 34, 579 (1962).

20. P. G. Shewmon, "Diffusion in Solids," McGraw-Hill Book Company, New York, N.Y., 1963, p. 30.

21. C. Baker and H. K. Birnbaum, Acta Meta11., 21, 865 (1973).

22. G. Pfeiffer and H. Wipf, J. Phys. F: Metal Phys., 6 , 167 (1967).

23. H. Buckle, Z. Metallkund, 37, 53 (1946).

24. P. G. Shewmon, "Diffusion in Solids," McGraw-Hill Book Company, New York, N.Y., 1963, p. 126.

25. Yu. K. Ivashina, V. F. Nemchenko and V. G. Charnetskiy, The Physics of Metals and Metallography (Eng. Trans.), $40,97(1975)$.

26. G. T. Meaden, "Electrical Resistance of Metals," Plenum Press, New York, N.Y., 1965, p. 16.

27. D. G. Westlake and S. T. Ockers, Metallurgical Trans., 6A, 399 (1975).

28. J. A. Pryde and I. T. Tsong, Acta Meta11., 19, 1333 (1971).

29. R. Kutner and I. Sosnowska, Acta Physica Polonica, A46, 755 (1974).

30. E. Veleckis and R. K. Edwards, J. Phys. Chem., 73, 683 (1969).

31. E. Lang and J. Bressers, Z. Metallkunde, 66, 619 (1976).

32. R. A. Pasternak and B. Evans, Trans. of the Metallurgical Society of AIME, 233, 1194 (1965).

33. S. H. Liu, private communication. Iowa State University, Ames, Iowa.

34. E. W. Montroll and R. B. Potts, Phys. Rev., 100, 525 (1955).

35. L. Nordheim, Ann. Phys., 9, 607 (1931). 
36. R. Berman, E. L. Foster and J. M. Ziman, Proc. Roy, Soc. A, 237, $344(1956)$.

37. J. M. Corsan and A. J. Cook, Phys. Status Solidi, 40, $657(1970)$. 
APPENDIX A. MAXIMUM EXPERIMENT TIMES

FOR ELECTRIC MOBILITY MEASUREMENTS

The validity of Equation 13, used in the calculation of the electromigration velocity, is subject to two restrictions which limit the maximum time over which $\mathrm{d} \Delta \mathrm{V} / \mathrm{dt}$ can be measured. First, the length of the experiment must not go beyond the time needed for significant movement of solute past the outer voltage probes. Secondly, there must be no appreciable decrease in the solute concentration near the central voltage probe. Experimentally, it is found that the distance hydrogen can diffuse is much greater than solute displacement due to electromigration in an equivalent time. Because of this, estimates of the maximum experiment time, $t_{\max }$, can be based on diffusion calculations alone.

By examination of the experimental dimensions shown in Figure 1 it can be seen that the diffusion distance for each restriction on $t_{\max }$ is equal. The distance from an outer knife-edge to the initial edge of the solute concentration profile is equal to the initial half-width of the concentration profile. It should be noted that until there is a decrease in solute concentration at the center of the solute profile, diffusion is proceeding on both ends of the profile as if from a source of infinite extent. Because $t_{\max }$ is defined as the time when a diffusional decrease begins to occur at the center of the solute profile, $t_{\text {max }}$ can be esti- 
mated from a calculation based on infinite source diffusion. When this assumption is made, $t_{\max }$ for both conditions on experiment length become equal due to the symmetry of the diffusion distances.

The basic equation governing diffusion from a source of concentration $c_{0}$ which is infinite in the $-x$ direction can be written as

$$
c(x, t)=\frac{c_{0}}{2}\left[1-\operatorname{erf} \frac{x}{2(D t)^{1 / 2}}\right]
$$

where $x=0$ corresponds to the original solute interface. Using the experimental diffusion distance for $t_{\max }$ as $1.0 \mathrm{~cm}$, the condition defining $t_{\max }$ will be chosen as: $t=t_{\max }$ when $c(x=1.0 \mathrm{~cm}, t)=0.01 c_{0}$. Using this definition, Equation A-1 can be solved for $t_{\max }$, yielding

$$
t_{\max }=\frac{1}{4 D}\left[\operatorname{erf}^{-1}(0.98)\right]^{-2} \text {. }
$$

Using diffusivities listed in Table 3 , maximum experiment times can be generated and are shown in Table A-1.

Table A-1. Maximum experiment times

\begin{tabular}{lcccccc}
\hline & $\mathrm{V}-\mathrm{H}$ & $\mathrm{V}-\mathrm{D}$ & $\mathrm{Nb}-\mathrm{H}$ & $\mathrm{Nb}-\mathrm{D}$ & $\mathrm{Ta}-\mathrm{H}$ & $\mathrm{Ta}-\mathrm{D}$ \\
\hline $\mathrm{t}_{\max }$ (minutes) & 25 & 75 & 150 & 340 & 620 & 1350 \\
\hline
\end{tabular}

Because of the reversal of electromigration direction near the mid-point of the experiment, the maximum displacement of the solute profile due to electromigration will be cqual 
to $v \cdot t_{\max } / 2$, where $v$ is the velocity of electromigration. Using an electric field of $5.5 \times 10^{-2}$ volts/cm and the average electric mobilities given in $T a b l e ~ 3$ to calculate $v, v \cdot t_{\text {max }} / 2$ can be shown to be near $0.1 \mathrm{~cm}$ for each metal-hydrogen isotope system. Thus, the assumption made in the calculation of $t_{\max }$ concerning the relative magnitude of diffusion distances and maximum electromigration displacements can be checked. The diffusion distances for the time interval, $t_{\max }$, are $1.0 \mathrm{~cm}$. This distance is large compared to the maximum electromigration distance of $0.1 \mathrm{~cm}$. Therefore, the calculation of $t_{\max }$ should be reasonably accurate. 


\section{APPENDIX B. ELECTROLYTIC INTRODUCTION \\ OF HYDROGEN ISOTOPES}

Electrolytic, or alternatively, cathodic introduction of hydrogen into Group VB metals offers several advantages which make electrolytic methods preferable to high temperature gas equilibrium techniques for this research. Electrolytic charging can be done quite rapidly at ambient temperatures with a minimum of special equipment. More importantly, regions of a sample can be selectively charged with hydrogen by allowing the electrolyte to contact the sample only where a hydrogen concentration is desired.

When a Group VB metal is used as a cathode in an electrolytic cell, nascent hydrogen can be adsorbed onto the surface after $\mathrm{H}^{+}$ions have been reduced near the surface of the specimen. From the surface, the adsorbed hydrogen atoms should diffuse inward producing a solute concentration in the bulk of the sample.

The amount of hydrogen absorbed is controlled by the number of coulombs which pass through the electrolytic cell. In theory, each electron which flows through the cell should be responsible for the introduction of one hydrogen atom into the specimen. However, some of the hydrogen atoms on, or near the surface of the sample, combine to form hydrogen gas molecules and thus, the efficiency of charging is never $100 \%$.

The surface condition of the sample appears to have a 
pronounced effect on the efficiency of electrolytic charging. It is necessary to electropolish the samples in order to provide a "clean" surface in order that the efficiency of charging and uniformity of solute distribution can be increased. Presumably, electropolishing removes any surface oxide layer which may impede the adsorption process. Niobium and tantalum. specimens were electropolished at 20 V.D.C. using a $7 \% \mathrm{H}_{2} \mathrm{SO}_{4}$ methyl alcohol electrolyte at $-60^{\circ} \mathrm{C}$ while vanadium samples required a $25 \% \mathrm{H}_{2} \mathrm{SO}_{4}$-methyl alcohol electrolyte at $-5^{\circ} \mathrm{C}$ and a potential of 12 V.D.C. The electropolishing time was usually less than 90 seconds to keep sample diameters uniform.

The electrolytes used for cathodic introduction of hydrogen isotopes were $10 \% \mathrm{H}_{2} \mathrm{SO}_{4}-\mathrm{H}_{2} \mathrm{O}$ or $10 \%(\mathrm{D}, \mathrm{H})_{2} \mathrm{SO}_{4}-\mathrm{D}_{2} \mathrm{O}$. The $\mathrm{H}_{2} \mathrm{O}$ and $\mathrm{D}_{2} \mathrm{O}$ were distilled from a potassium permanganate solution to oxidize any impurities which may interfere with the charging process. The $(\mathrm{D}, \mathrm{H})_{2} \mathrm{SO}_{4}$ was prepared by distilling $(\mathrm{D}, \mathrm{H})_{2} \mathrm{O}$ from a $15 \% \mathrm{H}_{2} \mathrm{SO}_{4}-\mathrm{D}_{2} \mathrm{O}$ solution. Mass spectrometric analysis of the gas removed by hot vacuum extraction from a sample charged in the deuterium-rich electrolyte indicated. that the fraction of hydrogen present was less than $2 \%$. Platinum was used as the anode for the electrolytic charging process to decrease the possibility of depositing metal ions, rather than hydrogen, at the cathode.

The cathode current densities used in this research were on the order of 2 to $3 \mathrm{amps} / \mathrm{cm}^{2}$. Charging at lower current 
densities appeared to yield lower charging efficiencies. Charging times ranged from 0.5 seconds to 30 seconds depending on sample size and desired solute concentration.

The observed charging efficiencies varied markedly with the metal being charged. The average hydrogen charging efficiencies, using room temperature electrolytes, were $75 \%$ for vanadium, $40 \%$ for niobium and $15 \%$ for tantalum. Although most charging was done at ambient temperatures, efficiency of charging, especially for tantalum, appeared to increase slightly when the electrolyte was heated to around $60^{\circ} \mathrm{C}$. The average deuterium charging efficiencies were about $10 \%$ lower for each metal.

Because of the isotopic and temperature dependence of the charging efficiency, as well as the large decrease in efficiency on going from vanadium to tantalum, it might well be postulated that the efficiency of charging is partly diffusion controlled. The diffusivity of hydrogen is generally a factor of two greater than the diffusivity of deuterium in a given Group VB metal. Also, as seen in Table 3 , the diffusivity of hydrogen isotopes decreases by a factor of 25 on going from vanadium to tantalum. The dependence of the charging efficiency on the solute diffusivity may be a reasonable result for the domain of high current densities since all the hydrogen is deposited on the surface of the sample within a few seconds. The number of available sites for hydrogen adsorp- 
tion may depend on how rapidly these sites can be cleared by the inward diffusion of previously deposited hydrogen.

It is possible to form a stable anodic layer on niobium and tantalum. This layer can be used to demarcate the area of the specimen exposed to the charging electrolyte, as well as to serve as a possible barrier to hydrogen desorption. The anodic layer can be produced by merely reversing the polarity of the electrolytic charging cell. The anodic layer is formed almost instantaneously upon application of the potential across the cell. Because the anodic layer is non-conductive, the current flowing through the cell quickly drops to near zero. Due to the insulating character of the anodic layer, care must be taken not to anodize any portion of the specimen to which electrical contact must be made. The color of the layer ranges from brown to purple, depending on the magnitude of the potential applied to the cell. Unfortunately, the anodic layer produced by this technique on vanadium is soluble in the electrolyte and methyl alcohol and does not adhere to the surface. 
APPENDIX C. TIME TO STEADY-STATE

Computer simulation of the steady-state electromigration experiment was used to determine the time required to produce steady-state solute concentration profiles. The simulation was based on the expression for the net flux of solute due to diffusion and electromigration at any location along the sample, Equation 19. The net change in solute concentration for a small portion of the sample between time $t$ and $t+\Delta t$ can be written as:

$$
\Delta c=\left(J_{\text {in }}-J_{\text {out }}\right) \cdot \Delta t / \ell
$$

where $J_{\text {in }}$ and $J_{\text {out }}$ are the solute fluxes into and out of the section and $l$ is the length of the small section.

For simulation, a rod was composed of a number of sma11 sections, each of which could exchange solute by diffusion and electromigration with its nearest neighbor. The flux through the outer-most boundaries of the segments was taken as zero for all time to create a system for which the total amount of solute was constant.

The number of sections needed to simulate a specimen of a given length is determined by the magnitude of non-linear solute concentration gradients which may be created within the sample. For purposes of estimating diffusional fluxes, the gradient in concentration existing in the $i^{\text {th }}$ section was calculated from a linear interpolation of the concentration assigned to the $i-1$ and $i+1$ sections. The validity of this 
approximation decreases as the change in concentration between segments increases in a non-linear fashion. Therefore, if a large, non-1inear concentration gradient exists, a greater number of sections is required to accurately represent that gradient and to correctly calculate the diffusive contribution to the net solute flux. For steady-state concentration profiles in which the end segment concentrations differed by less than a factor of three, 32 sections were found to adequately represent a $4.0 \mathrm{~cm}$ sample.

Initially, all segments are given equal solute concentration. After a time interval, $\Delta t$, the concentration of each segment will be changed by an amount, $\Delta c$, calculated for each segment from Equation C-1. After up-dating the concentration in each segment, new diffusive and electromigration fluxes can be calculated for the time interval from $\Delta t$ to $2 \Delta t$. This process can be continued until $\Delta c$ for each segment of the sample is near zero. The solute profile should now show an exponential dependence, as predicted by Equation 22 for a system at steady-state.

There is a maximum value for the iteration time, $\Delta t$, which if exceeded, will produce erroneous results. The existence of a maximum iteration time can be understood by examination of the assumption in the simulation that any flux out of the $i^{\text {th }}$ section can only affect the concentration of the $i-1$ or $i+1$ section. For example, $\Delta t$ may be chosen such that 
solute atoms in the $i^{\text {th }}$ section would have sufficient time to produce concentration changes at a distance corresponding to the $i+1, i+2$ and $i+3$ sections. In this case, the assumption that all diffusive flux from the $i^{\text {th }}$ section will only change the concentration of the $i+1$ section will be invalid and the concentration increase calculated for the $i+1$ section will be much too large. Therefore, the value of $\Delta t$ used must be small enough to limit the range of solute motion to a single segment during that iteration. Mathematically speaking, $\Delta t$ must be chosen such that

$$
2(D \cdot \Delta t)^{1 / 2}<\ell
$$

and

$$
v \cdot \Delta t<\ell
$$

where $l$ is the length of a section, $v$ is the velocity of electromigration and $\mathrm{D}$ is the solute diffusivity.

The definition of the time needed to achieve steady-state conditions is arbitrary. For this simulation, the time required to attain steady-state was defined as the time at which the inequality

$$
\left|\frac{c-c s s}{c_{i}-c_{s s}}\right|<0.05
$$

was satisfied for the end points of the specimen and two other segments spaced uniformly along the rod. $c$ is the simulation concentration at a given time, $c_{s s}$ is the true steady-state concentration predicted by Equation 22 and $c_{i}$ is the initial concentration of the specimen. 
Input data for the velocity of electromigration and solute diffusivity for the pure metals was obtained from the measured electric mobility and the diffusion data listed in Table 3. For the niobium-tantalum alloys, a linear interpolation was used to estimate $v$ and $D$. In retrospect, this turned out to be quite an accurate estimate.

The results listed in Tables $C-1$ and $C-2$ can be used to determine the factors controlling the time to steady-state. As can be seen in $\mathrm{Table} \mathrm{C}-1$, the time required to reach steady-state appears to be independent of the electromigration velocity and $v / D$. However, as shown in Table $\mathrm{C}-2$ the quantity $\ell /\left(D t_{s s}\right)^{1 / 2}$ is relatively constant for a wide range of diffusivities, electromigration velocities and sample lengths. Therefore, it appears as if the time to reach steady-state conditions is diffusion controlled for the range of velocities and diffusivities simulated. Thus, when considering the time needed for a specimen containing hydrogen and deuterium to attain steady-state, $t_{s s}$ should be calculated from the diffusivity of deuterium, since it will be the last isotope to reach steady-state. 
Table C-1. Time to steady-state required for several diffusivities and velocities of transport in an $8.0 \mathrm{~cm}$ sample

\begin{tabular}{|c|c|c|c|}
\hline $\begin{array}{l}\text { Velocity } \times 10^{6} \\
\mathrm{~cm} / \mathrm{sec}\end{array}$ & 1.45 & 2.9 & 5.8 \\
\hline $\mathrm{D}=1.05 \times 10^{5} \mathrm{~cm}^{2} / \mathrm{sec}$ & 24 days $^{a}$ & 24 days $^{b}$ & -. \\
\hline $\mathrm{D}=2.1 \times 10^{5} \mathrm{~cm}^{2} / \mathrm{sec}$ & 14 days $^{c}$ & 14 days $^{a}$ & 14 days $^{b}$ \\
\hline $\mathrm{D}=4.2 \times 10^{5} \mathrm{~cm}^{2} / \mathrm{sec}$ & -. & 7 days $^{c}$ & 7 days $^{a}$ \\
\hline $\begin{array}{l}\mathrm{a}_{\mathrm{v} / \mathrm{D}}=0.15 \mathrm{pe} \\
\mathrm{b}_{\mathrm{v} / \mathrm{D}}=0.28 \mathrm{pe} \\
\mathrm{c}_{\mathrm{v} / \mathrm{D}}=0.07 \mathrm{pe}\end{array}$ & $\mathrm{m}$ & & \\
\hline
\end{tabular}

Table C-2. $\quad l /\left(D t_{s s}\right)^{1 / 2}$ for several diffusivities, velocities of migration and specimen lengths.

\begin{tabular}{cccc}
\hline $\begin{array}{c}\mathrm{D} \times 10^{5}, \\
\mathrm{~cm}^{2} \mathrm{sec}\end{array}$ & $\ell, \mathrm{cm}$ & $\mathrm{t}_{\mathrm{s.s}}$, days & $\ell /(\mathrm{Dt})^{1 / 2}$ \\
\hline 1.05 & 8.0 & 24 & 1.6 \\
2.10 & 8.0 & 14 & 1.6 \\
0.09 & 5.0 & 91 & 1.8 \\
0.49 & 4.0 & 22 & 1.4 \\
0.60 & 4.0 & 15 & 1.4 \\
\hline
\end{tabular}

